Prepared in cooperation with Douglas County and the U.S. Forest Service

Water Quality and Algal Conditions in the North Umpqua River, Oregon, 1995-2007, and their Response to Diamond Lake Restoration
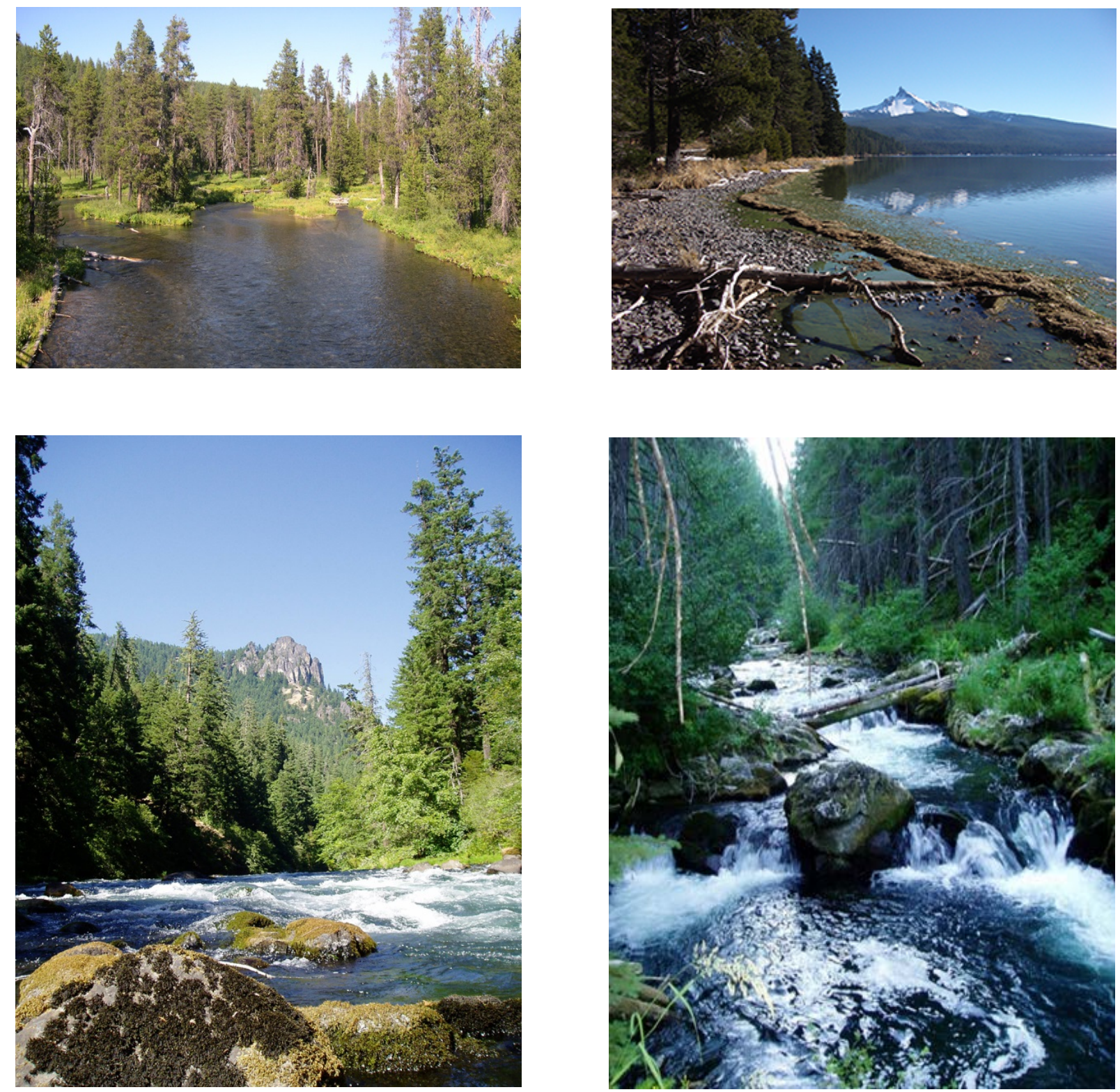

Open-File Report 2014-1098

U.S. Department of the Interior

U.S. Geological Survey 
Cover: Clockwise from upper left - (1) Upper North Umpqua River at Inlet Campground, (2) Diamond Lake with Mt. Thielsen in the background, (3) Wild and Scenic River reach downstream from Copeland Creek, (4) middle North Umpqua River downstream of Lemolo Lake. (Photographs 1, 3, and 4 by Kurt Carpenter, U.S. Geological Survey; photograph 2 by Mikeal Jones, U.S. Forest Service, ret.) 


\section{Water Quality and Algal Conditions in the North Umpqua River, Oregon, 1995-2007, and their Response to Diamond Lake Restoration}

By Kurt D. Carpenter, Chauncey W. Anderson, and Mikeal E. Jones

Prepared in cooperation with Douglas County and the U.S. Forest Service

Open-File Report 2014-1098

U.S. Department of the Interior

U.S. Geological Survey 


\section{U.S. Department of the Interior \\ SALLY JEWELL, Secretary}

\section{U.S. Geological Survey \\ Suzette M. Kimball, Acting Director}

U.S. Geological Survey, Reston, Virginia 2014

For more information on the USGS-the Federal source for science about the Earth, its natural and living resources, natural hazards, and the environment, visit http://www.usgs.gov_or call 1-888-ASK-USGS.

For an overview of USGS information products, including maps, imagery, and publications, visit $h$ ttp://www.usgs.gov/pubprod

To order this and other USGS information products, visit $h$ ttp://store.usgs.gov

\section{Suggested citation:}

Carpenter, K.D., Anderson, C.W., and Jones, M.E., 2014, Water quality and algal conditions in the North Umpqua River, Oregon, 1995-2007, and their response to Diamond Lake restoration: U.S. Geological Survey Open-File Report 2014-1098, 89 p. http://dx.doi.org/10.3133/ofr20141098.

ISSN 2331-1258 (online)

Any use of trade, product, or firm names is for descriptive purposes only and does not imply endorsement by the U.S. Government.

Although this report is in the public domain, permission must be secured from the individual copyright owners to reproduce any copyrighted material contained within this report. 


\section{Contents}

Summary

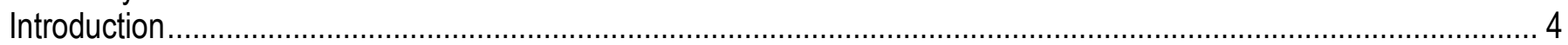

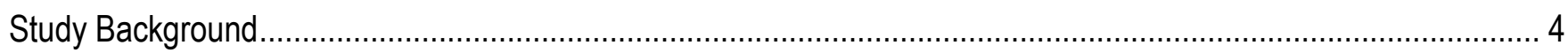

Study Objectives, Approach, and Report Scope ……………..................................................................... 8

Study Area

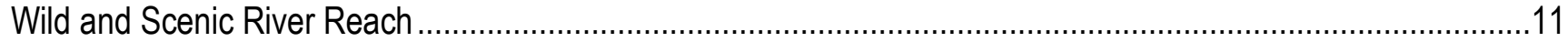

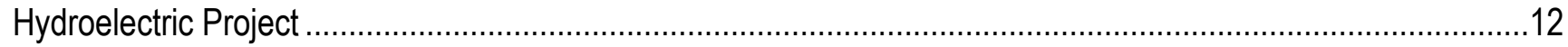

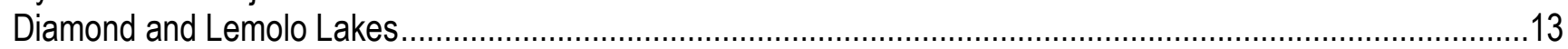

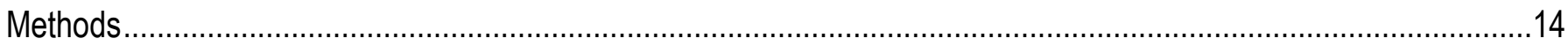

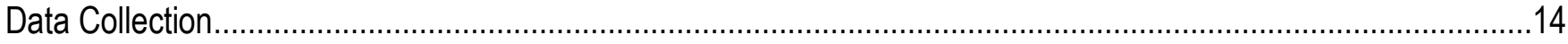

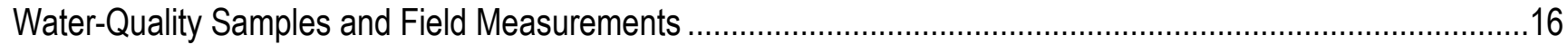

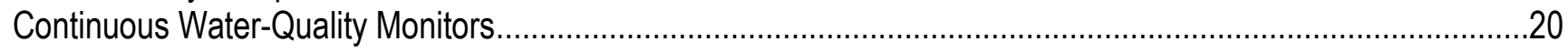

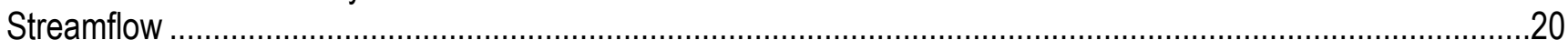

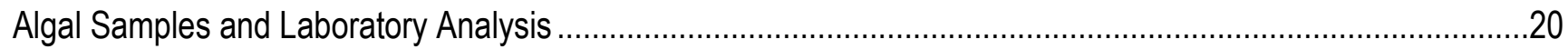

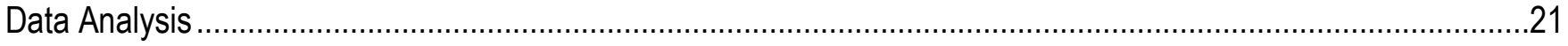

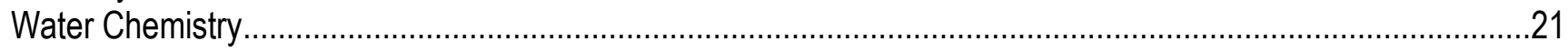

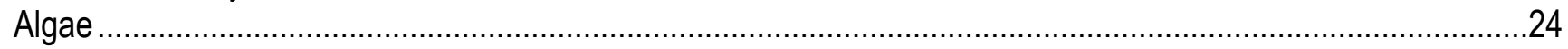

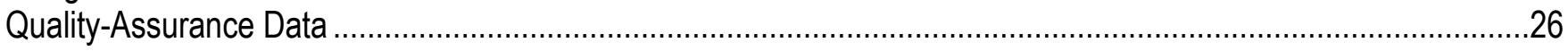

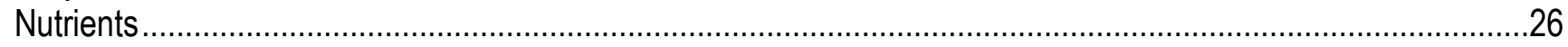

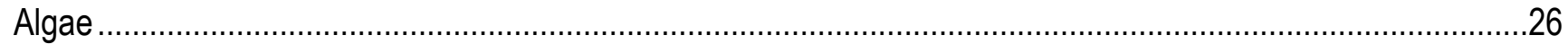

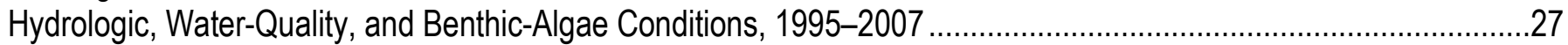

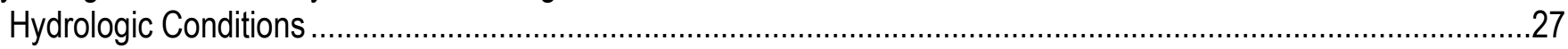

Patterns in Hydrology in 1995-2007 and Changes Associated with the New Hydroelectric License ...................27

Changes in Hydrology Associated with the Diamond Lake Restoration Project.................................................32

Water-Quality Conditions .............................................................................................................................

Field Parameters_-Water Temperature, Dissolved Oxygen, pH, and Turbidity ..............................................33

Lake Creek and the Diamond Lake Drawdown .........................................................................................4

Nutrients and Organic Carbon ........................................................................................................4

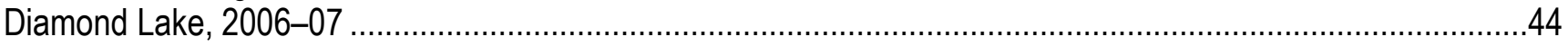

Monthly Sampling in Lake Creek and the North Umpqua River, 2006-07....................................................44

Comparison of Nutrient Concentrations and Algal Biomass to Suggested Criteria..............................................50

Changes in Water-Quality Conditions between 1995 and 2005-07 ……...................................................50

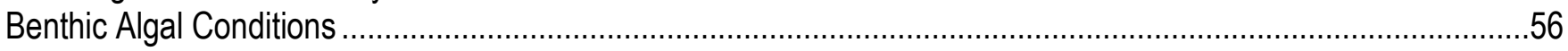

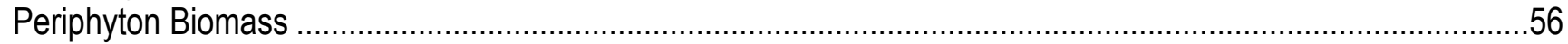

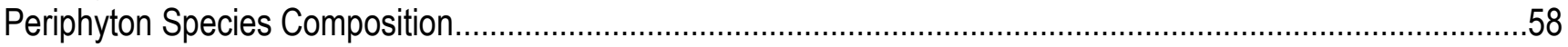

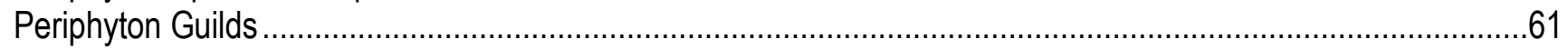

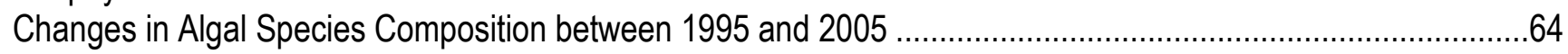

Changes in Algal Species Composition between 2005 and 2007 ..........................................................66

Algal Conditions at Selected Sites from 1995 to 2005-07 ...................................................................

Factors Shaping Periphyton Assemblages: Results of Multivariate BEST Analyses.........................................70

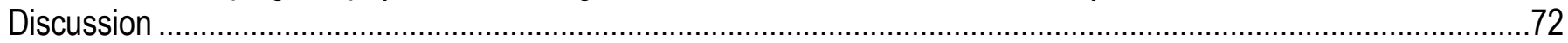

Effects of the Diamond Lake Restoration Project on Lake Creek and the North Umpqua River ...............................72

Streamflow, Water Quality, and Algal Conditions between Synoptic Surveys.....................................................73

Potential Effects of Resource Management on Water Quality and Algal Conditions.............................................76

Suggestions for Future Monitoring and Studies ......................................................................................... 
Conclusions.

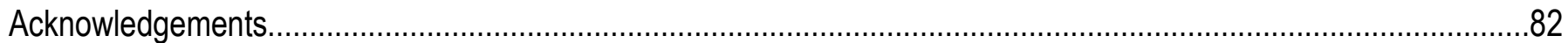

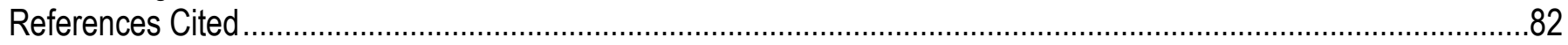

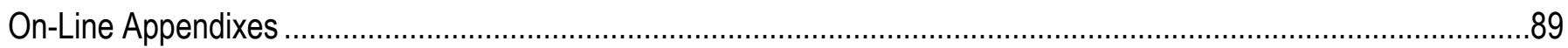

Appendix A. Laboratory Detection Limits and Reporting Levels ......................................................................8

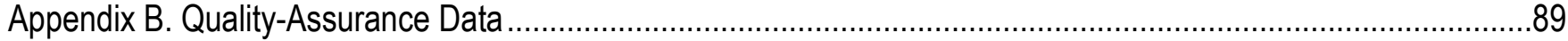

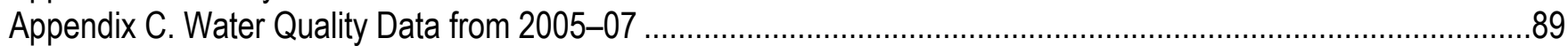

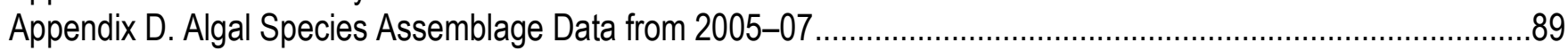

\section{Figures}

Figure 1. Map of the North Umpqua River basin showing the sampling-site locations. 5

Figure 2. Schematic diagram of the upper North Umpqua River basin, Oregon, showing the complex routing of water through the Hydroelectric Project.

Figure 3. Graphs showing streamflow in the North Umpqua River above Copeland Creek (COPEG), Oregon, for (A) water year 1993-2007 and (B) summers of 1995 and 2005-07. 10

Figure 4. Streamflow in Lake Creek, Diamond Lake outflow canal, and total combined flow during and after the Diamond Lake drawdown, December 2005 to October 2007 ........................................................ 32

Figure 5. Selected diel field parameter values for the (A) main-stem North Umpqua River and (B) tributaries during the 1995, 2005, and 2007 synoptic surveys. See table 5 for site code definitions.

Figure 6. Continuous field parameter data from North Umpqua River near Idleyld Park (14137450), Oregon, 2005-07, showing (A) the 7-day average of the daily maximum water temperatures and (B) daily minimum dissolved oxygen...

Figure 7. Diel patterns in water temperature and dissolved oxygen in the North Umpqua River near Idleyld Park, July 28-August 2, 2005 .

Figure 8. (A) River stage and pH during the August 2007 synoptic survey and (B) daily maximum pH and daily minimum dissolved oxygen concentration in the North Umpqua River at the inflow to Toketee Lake (TOKLKI), May-September 2007.

Figure 9. Streamflow and (A) daily mean specific conductance and (B) daily mean water temperature, from August 2006 to September 2007..

Figure 10. Monthly nitrogen concentrations in Lake Creek and the downstream North Umpqua River during the Diamond Lake restoration project, 2005-07.

Figure 11. Monthly phosphorus concentrations in Lake Creek and the downstream North Umpqua River during the Diamond Lake restoration project, 2005-07.

Figure 12. Monthly carbon concentrations in Lake Creek and the downstream North Umpqua River during the Diamond Lake restoration project, 2005-07.

Figure 13. Nutrient and carbon ratios for sites sampled monthly in the North Umpqua River basin during the Diamond Lake restoration project, 2005-07.

Figure 14. Concentrations of soluble reactive phosphorus and total phosphorus in the (A) main-stem North Umpqua River and (B) tributaries during the 1995, 2005, and 2007 synoptic surveys.

Figure 15. Longitudinal patterns in concentrations of dissolved inorganic nitrogen and total nitrogen in the (A) main-stem North Umpqua River and (B) tributaries during the 1995, 2005, and 2007 synoptic surveys. 53

Figure 16. Streamflow and loads of nitrogen and phosphorus in the (A) main-stem North Umpqua River and (B) tributaries during the 1995, 2005, and 2007 synoptic surveys.

Figure 17. Patterns in (A) periphyton biomass indicators and (B) percent biovolume of selected algal-indicator guilds in Lake Creek and the North Umpqua River during the 1995, 2005, and 2007 synoptic surveys..57

Figure 18. Percent biovolume of algal-indicator guilds in Lake Creek and the North Umpqua River during the 1995,2005 , and 2007 synoptic surveys.. 
Figure 19. Percent biovolume of green algae in Lake Creek and the North Umpqua River during the 1995, 2005, and 2007 synoptic surveys..

Figure 20. Ordination diagrams of algae samples from the North Umpqua River basin, Oregon, based on diatoms and soft algae for (A) samples collected in 1995 and 2005, and (B) samples collected in 2005-07......65

Figure 21. Algal conditions in the Diamond Lake Restoration Affected Reach, Lake Creek and the North Umpqua River, 1995, 2005-07, including (A) chlorophyll-a and ash-free dry mass, (B) select algal biovolume indicators, and $(\mathrm{C})$ percent biovolume of eutrophic algae.

\section{Tables}

Table 1. History of blue-green algae blooms and associated human-health advisories and management actions in Diamond and Lemolo Lakes, North Umpqua River basin, Oregon, 2001-10.....

Table 2. Timeline of data collection and events related to the rotenone application in Diamond Lake

Table 3. Minimum in-stream flows for bypass reaches of the North Umpqua River Hydroelectric Project required prior to and following issuance of the new Federal Energy Regulatory Commission license, settlement agreement, and 401 Water-Quality Certification.

Table 4. Field parameter data collection and long term continuous monitor deployment periods for sites in the North Umpqua River basin, 2005-07

Table 5. Water quality and algae sampling sites in the North Umpqua River basin, Oregon, 1995-2007

Table 6. State of Oregon water quality standards (numeric criteria) for temperature, dissolved oxygen, and $\mathrm{pH}$ for indicated reaches in the North Umpqua River basin, Oregon.

Table 7. Concentrations suggested for reference streams and as criteria for nutrients and algal biomass to prevent nuisance conditions in the North Umpqua River basin, Oregon.

Table 8. Comparisons of flow-duration statistics for the North Umpqua River above Copeland Creek for 19902000 and $2000-10$.

Table 9. Site-specific streamflow conditions during the summer synoptic sampling events in the North Umpqua River basin, Oregon, 1995, 2005, and 2007.

Table 10. Average percent change in streamflow, select field parameters, and nutrients at sites located in the Wild and Scenic Reach, tributaries, and the Diamond Lake Restoration Affected Reach, for 1995-2005 and 20052007.

Table 11. Distribution of major algal taxonomic divisions in samples from the North Umpqua River Basin, Oregon, 1995 and 2005-07.

Table 12. Top five most abundant algal taxa based on percent biovolume for selected sites in the North Umpqua River basin, Oregon, 1995 and 2005-07.

Table 13. Description of periphyton indicator-species guilds identified from samples collected in the North Umpqua River basin, Oregon, 1995 and 2005-07.

Table 14. Periphyton indicator-species guild values for samples collected from the North Umpqua River basin, Oregon, 1995 and 2005-07.

Table 15. Average percent biovolume of select algal taxa at sites extending from Lake Creek to the North Umpqua River above Rock Creek, 1995, 2005, and 2007.

Table 16. Summary of BEST analysis solutions listing the measured variables that best explain patterns in periphyton assemblages for sites within the Diamond Lake Restoration Affected Reach extending from Lake Creek to the North Umpqua River above Rock Creek, 1995, 2005, and 2007. 


\section{Conversion Factors, Abbreviations and Acronyms, and Site Codes}

\section{Conversion Factors}

\begin{tabular}{lll}
\hline \multicolumn{1}{c}{ Multiply } & \multicolumn{1}{c}{ By } & \multicolumn{1}{c}{ To obtain } \\
\hline micrometer $(\mu \mathrm{m})$ & 0.00004 & inch (in) \\
cubic micrometer $\left(\mu \mathrm{m}^{3}\right)$ & $6.10237 \times 10^{-14}$ & cubic inch $\left(\mathrm{in}^{3}\right)$ \\
millimeter $(\mathrm{mm})$ & 0.03937 & inch (in) \\
centimeter $(\mathrm{cm})$ & 0.3937 & inch (in) \\
square centimeter $\left(\mathrm{cm}^{2}\right)$ & 0.1550 & square inch $\left(\mathrm{in}^{2}\right)$ \\
square meter $\left(\mathrm{m}^{2}\right)$ & 10.76 & square foot $\left(\mathrm{ft}^{2}\right)$ \\
milligram & 0.03527 & ounce, avoirdupois $(\mathrm{oz})$ \\
milliliter $(\mathrm{mL})$ & 0.00382 & ounce, fluid $(\mathrm{fl} . \mathrm{oz})$ \\
liter $(\mathrm{L})$ & 33.82 & ounce, fluid $(\mathrm{fl} . \mathrm{oz})$ \\
liter $(\mathrm{L})$ & 1.057 & quart $(\mathrm{qt})$ \\
cubic foot per second $\left(\mathrm{ft}^{3} / \mathrm{s}\right)$ & 0.02832 & cubic meter per second $\left(\mathrm{m}^{3} / \mathrm{s}\right)$ \\
\hline
\end{tabular}

Temperature in degrees Celsius $\left({ }^{\circ} \mathrm{C}\right)$ may be converted to degrees Fahrenheit $\left({ }^{\circ} \mathrm{F}\right)$ as follows:

$$
{ }^{\circ} \mathrm{F}=\left(1.8 x^{\circ} \mathrm{C}\right)+32
$$

Temperature in degrees Fahrenheit $\left({ }^{\circ} \mathrm{F}\right)$ may be converted to degrees Celsius $\left({ }^{\circ} \mathrm{C}\right)$ as follows:

$$
{ }^{\circ} \mathrm{C}=\left({ }^{\circ} \mathrm{F}-32\right) / 1.8
$$

Vertical coordinate information is referenced to the North American Vertical Datum of 1988 (NAVD 88).

Horizontal coordinate information is referenced to the North American Datum of 1983 (NAD 83).

Altitude, as used in this report, refers to distance above the vertical datum.

Specific conductance is given in microsiemens per centimeter at 25 degrees Celsius $\left(\mu \mathrm{S} / \mathrm{cm}\right.$ at $\left.25^{\circ} \mathrm{C}\right)$.

Concentrations of chemical constituents in water are given in milligrams per liter $(\mathrm{mg} / \mathrm{L}$, approximately equivalent to parts per million [ppm]) or micrograms per liter ( $\mu \mathrm{g} / \mathrm{L}$, approximately equivalent to parts per billion [ppb]).

Algal biomass values are given in milligrams per square meter $\left(\mathrm{mg} / \mathrm{m}^{2}\right)$.

Algal biovolume values are given in cubic micrometers per square centimeter $\left(\mu \mathrm{m}^{3} / \mathrm{cm}^{2}\right)$. 


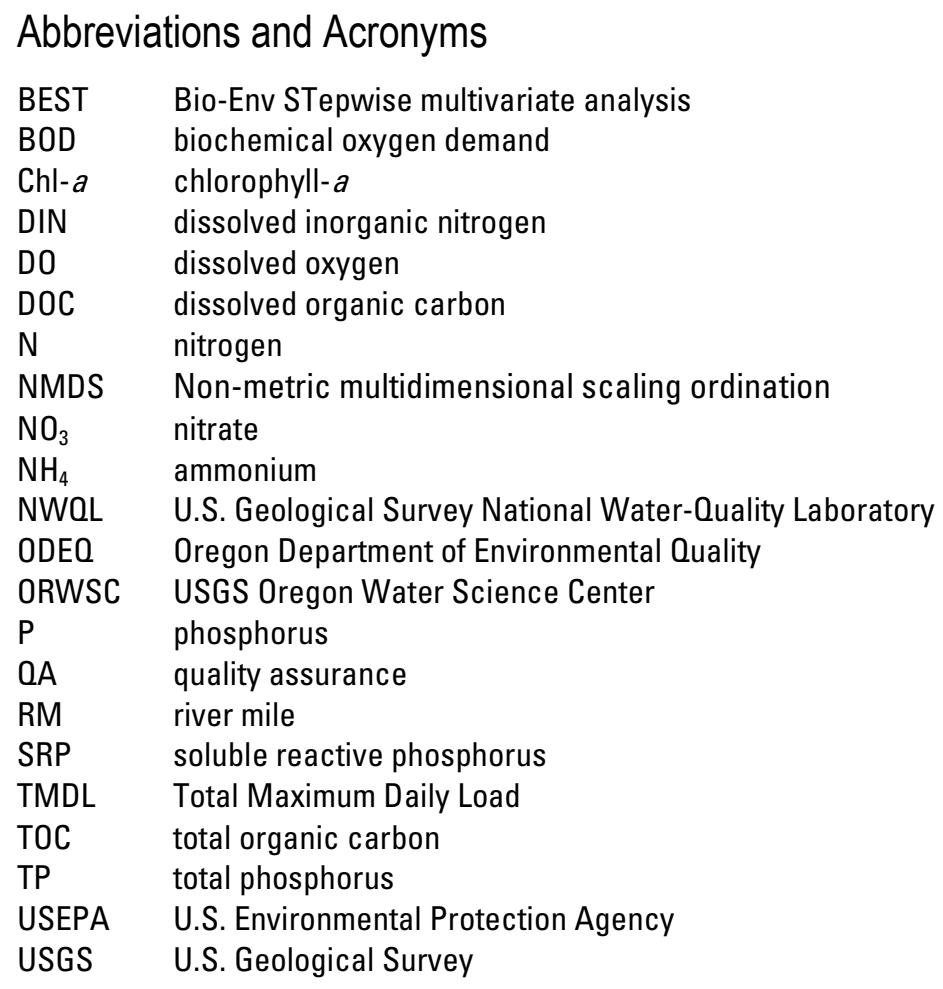

\section{Site Codes}

BOULM Boulder Creek near mouth

BSODA North Umpqua River below Soda Springs powerhouse

CALFM Calf Creek near mouth

CANTM Canton Creek near mouth

CLR2B Clearwater Canal \#2 bypass reach

CLRAS Clearwater River above Stump Lake

COPEG North Umpqua River above Copeland Creek

COPEM Copeland Creek near mouth

INLET North Umpqua River above Lemolo Lake near Inlet campground

LAKE138 Lake Creek downstream of Diamond Lake at Highway 138

LAKEM Lake Creek above Lemolo Lake

LEM1T/G Lemolo\#1 bypass reach at the gage (G) or Top (T) of bypass reach

LITGL Little River near Glide

MOTTB North Umpqua River at Mott Bridge

NUAPC North Umpqua River at Apple Creek

NUBWC North Umpqua River below Wright Creek

NUGLD North Umpqua River near Glide

NURCR North Umpqua River above Rock Creek

NUSTM North Umpqua River near Steamboat

PANTM Panther Creek near mouth

ROCKM Rock Creek near mouth

SODAT North Umpqua River below Soda Springs

STEABB Steamboat Creek above Big Bend Creek

STEAM Steamboat Creek at gage

SUSNM Susan Creek near mouth

TOKET North Umpqua River below Toketee Lake

TOKLKI North Umpqua River above Toketee Lake

WRCRM Wright Creek near mouth 
This page is intentionally blank 


\title{
Water Quality and Algal Conditions in the North Umpqua River, Oregon, 1995-2007, and their Response to Diamond Lake Restoration
}

\author{
By Kurt D. Carpenter ${ }^{1}$, Chauncey W. Anderson ${ }^{1}$, and Mikeal E. Jones ${ }^{2}$
}

\section{Summary}

The Wild and Scenic North Umpqua River is one of the highest-quality waters in the State of Oregon, supporting runs of wild salmon, steelhead, and trout. For many years, blooms of potentially toxic blue-green algae in Diamond and Lemolo Lakes have threatened water quality, fisheries, and public health. The blooms consist primarily of Anabaena, a nitrogen (N)-fixing planktonic alga that appears to have contributed to $\mathrm{N}$ enrichment, which could account for changes in communities and biomass of periphyton, or attached benthic algae, in the river. Periphyton can become a nuisance in summer by affecting riffle habitat and causing high $\mathrm{pH}$ that fails to meet State of Oregon waterquality standards. These symptoms of nutrient enrichment in the North Umpqua River were first documented in 1995, and the symptoms have continued since then. Restoring natural ecosystem processes that store nutrients rather than fueling algae might help improve $\mathrm{pH}$ and water-clarity conditions.

This report summarizes the results from a study in 2005-07 characterizing water quality and algal conditions in the North Umpqua River before, during, and after the 2006 rotenone treatment of Diamond Lake in the headwaters of the North Umpqua River. The treatment was part of a restoration project to eradicate Tui chub (Gila bicolor), a non-native invasive fish blamed for decimating the trout fishery in Diamond Lake and fueling Anabaena blooms. Diamond Lake

\footnotetext{
${ }^{1}$ U.S. Geological Survey

${ }^{2}$ U.S. Forest Service
}

was expected to contribute organic matter and associated nutrients to Lake Creek during the project, but it was unclear whether these nutrients would affect periphyton communities in the North Umpqua River downstream.

This study also provided an opportunity to examine changes in stream conditions in the main stem North Umpqua River and its tributaries, which were previously sampled in July 1995. The 1995 study was designed to provide background data during relicensing of the upstream hydroelectric facilities, and was partly motivated by anecdotal concerns about increase periphyton growth and reduced water clarity. As part of the 2005-07 study associated with the Diamond Lake restoration project, we repeated the 1995 basinwide synoptic survey in 2005, before the rotenone treatment. Although both samplings were just a snapshot of conditions, these data were evaluated for possible changes between 1995 and 2005.

Less-than-average snowpack in 2005 resulted in lower-than-average streamflow in the tributaries and in the main stem than in 1995, which influenced water quality by reducing dilution. Concentrations of $\mathrm{N}$, phosphorus (P), and conductance were notably higher in the tributaries entering the Wild and Scenic Reach of the North Umpqua River in 2005 compared with 1995. Nitrate-N in the tributaries was consistently detected in 2005; in contrast, concentrations of nitrate- $\mathrm{N}$ were mostly less than the laboratory reporting level in 1995. Even small increases in $\mathrm{N}$ availability could fuel or change periphyton communities and cause 
changes in habitat and food resources for benthic invertebrates in this Wild and Scenic River.

In 1995 and 2005, periphyton biomass was highly variable, with areas of heavy growth downstream from hydroelectric project reservoirs and powerhouses, and low-to-nuisance biomass levels in Lake Creek and in the Wild and Scenic Reach downstream. From 1995 to 2005, periphyton biomass increased more than five-fold in Lake Creek near the mouth, which probably indicates greater $\mathrm{N}$ concentrations derived from blooms of $\mathrm{N}$-fixing blue-green algae in Diamond Lake. Compared with 1995, periphyton biomass in 2005 was 60 percent higher at the two main-stem sites downstream from Steamboat Creek, but remained below commonly referenced nuisance biomass levels.

Differences in the periphyton species compositions between 1995 and 2005 included greater abundances of filamentous green algae (primarily Cladophora glomerata) in the upper basin, and N-fixing diatoms (Epithemia spp.) in the Wild and Scenic Reach. Cladophora requires high $\mathrm{N}$ and $\mathrm{P}$ concentrations, and $\mathrm{P}$ tends to be ample in those parts of the basin, suggesting that the increases in dissolved $\mathrm{N}$ may have contributed to these biomass increases. Melosira varians, a diatom known to respond to greater $\mathrm{N}$ availability, was also relatively more abundant in 2005 compared with 1995.

The drawdown of Diamond Lake began in March 2006, prior to the rotenone application. As the lake refilled following the application, outflow from Lake Creek was lower than normal, 0-20 cubic feet per second. When Diamond Lake refilled in March 2007, Lake Creek resumed a typical springtime flow of about 50 cubic feet per second. The drawdown caused a substantial export of organic matter and associated $\mathrm{N}$ and $\mathrm{P}$ from Diamond Lake into Lake Creek and downriver. During the lake drawdown, periphyton biomass in Lake Creek in 2006 exceeded nuisance biomass levels, more than doubling that found in 2005. Although $\mathrm{P}$ concentrations in Lake Creek increased during the drawdown, the highest $\mathrm{P}$ concentrations occurred when flows from the lake stopped and groundwater dominated flow. The drawdown increased $\mathrm{N}$ and $\mathrm{P}$ concentrations in the Wild and Scenic Reach in 2006, but these increases were moderate, and attenuated downstream.

The restoration project increased the concentrations of $\mathrm{N}$ in Lake Creek in spring 2007 following the rotenone application. In March, when discharge from Diamond Lake resumed, $\mathrm{N}$ concentrations in Lake Creek increased, peaking at 0.5 milligrams per liter total $\mathrm{N}$ and nearly 0.2 milligrams per liter ammonium-N. By May 2007, the ammonium-N concentration in Lake Creek decreased to less than the laboratory reporting level. Compared with 2005, the 2007 average nitrate-N and total $\mathrm{P}$ concentrations in the Wild and Scenic Reach were 61 and 17 percent higher, respectively, after flow resumed from Diamond Lake.

Periphyton biomass in Lake Creek in August 2007 exceeded the nuisance level and eutrophic types of algae continued to dominate the assemblage at this site. Among the noted algal species shifts in Lake Creek was an increase in the stalked diatom Gomphoneis herculeana in 2007. This diatom taxon is similar in form to Didymosphenia, commonly known as "rock snot," which can produce high biomass, negatively affecting habitat for fish and other aquatic life in other streams by smothering river cobbles.

One effect of excessive periphyton growth is elevated $\mathrm{pH}$ in the late afternoon from photosynthesis. Since 1994, pH in the lower Wild and Scenic Reach has exceeded the waterquality standard of 8.5 units during most summers, including 2006 and 2007, with maximum values of 8.6 and 9.2 units, respectively. In 2007, the maximum $\mathrm{pH}$ exceeded the 8.5 standard for all of July and August and much of September, indicating greater biomass that year.

The Diamond Lake restoration project resulted in a measurable release of organic 
matter and nutrients into Lake Creek and the Wild and Scenic Reach downstream. The effect was transient, but the increase in nutrients contributed to increases in periphyton biomass and shifts in species composition in Lake Creek and perhaps other sites downstream. Although the restoration project increased nutrient concentrations downstream, modestly higher $\mathrm{N}$ concentrations were already apparent in the tributaries and Wild and Scenic Reach in 2005, prior to the restoration project. The cause(s) of this apparent increase remains unexplained.

There are several potential sources of $\mathrm{N}$ in the North Umpqua River Basin. N-fixing bluegreen algae in Diamond Lake are a probable source for observed $\mathrm{N}$ increases in Lake Creek, in addition to the post-rotenone decomposition of fish biomass that occurred during this study. Contributions from Lemolo Lake, from various possible mechanisms including blooms of Nfixing algae, also appear to enrich $\mathrm{N}$ levels in downstream waters. More difficult to explain is the source of nitrate- $\mathrm{N}$ in the tributaries, where concentrations were notably higher in 2005 than in 1995. Possible N sources include groundwater, atmospheric deposition, $\mathrm{N}$ fixed by algae and red alder trees, and decomposition of plant materials, including stands of lodgepole pine in the upper North Umpqua River Basin where outbreaks of mountain pine beetles (Dendroctonus ponderosae) have occurred.

Continued phytoplankton blooms and proliferation of periphyton and their effects on water quality and habitat in this Wild and Scenic River are a management challenge. The effects of high $\mathrm{pH}$ on aquatic life are not well understood, but could include impacts to the sensitive early life stages of salmonids and other fish. Maintaining the historically excellentquality water in the Wild and Scenic Reach of the North Umpqua River depends at least partly on the water quality and algal conditions in Diamond and Lemolo Lakes, making nutrient and (or) bloom reduction strategies critical for achieving this goal.
These aquatic conditions and underlying processes have important management implications for the North Umpqua River Basin. Looking forward, the continued operation of streamgages and water-quality monitors can provide the data framework to track conditions and detect possible trends. These data are critical for identifying problems, providing baseline information for comparison, and evaluating the success of potential management strategies. Future investigations could identify sources of nutrients fueling algal growth, examine the effects of high $\mathrm{pH}$ on salmon and steelhead fry, and measure the effect of forest health on nutrient retention in upper-basin forests. Flow characteristics within the hydroelectric project could also be further evaluated for their effects on stream conditions. And continued monitoring of Diamond and Lemolo Lakes, which has occurred since 2007, continue to provide information on bloom conditions over time and will add to our understanding of why blooms develop and what land and water managers might do to improve conditions. 


\section{Introduction}

The North Umpqua River (fig. 1) flows westward from the Oregon Cascades to its confluence with the South Umpqua River, and, from there, as the Umpqua River, to the Pacific Ocean. The river is known for its scenic beauty, outstanding recreational opportunities, and importance as a natural resource. Designated beneficial uses include public and private domestic water supply; industrial, irrigation, and livestock use; anadromous fish passage, spawning, and rearing; habitat for resident fish and aquatic life; fishing; boating; water contact recreation; aesthetics and hydropower (Oregon Administrative Rules, Chapter 340-41-0320). Most of the North Umpqua River Basin is either public or private forestland, and historically, large quantities of timber have been produced in the basin.

North Umpqua River water quality is excellent by many measures, but since at least the early 1990s, signs of eutrophication, or nutrient enrichment, have been apparent. The upper North Umpqua River Basin includes PacifiCorp's hydroelectric project and, downstream, the Wild and Scenic River (cover photograph 3). Anderson and Carpenter (1998) determined that the river was highly sensitive to inputs of nitrogen $(\mathrm{N})$, which is likely a limiting nutrient for periphyton (attached benthic algae) growth and productivity. The 1998 report also noted that daily maximum $\mathrm{pH}$ was elevated at some sites and at times did not meet the State of Oregon $\mathrm{pH}$ standard of 8.5 units, even in segments of the Wild and Scenic Reach. Since 2000, the U.S. Geological Survey (USGS) has operated a continuous water-quality monitor at the downstream end of the Wild and Scenic Reach near Idleyld Park (upstream of Rock Creek, at river mile [RM] 35.8). From 2000 to 2010 , the daily maximum $\mathrm{pH}$ values exceeded 8.5 units on nearly 40 percent of the days during June-August (U.S. Geological Survey, 2014a).
A draft assessment by the Oregon Department of Environmental Quality (ODEQ) (2010) listed the following waters as waterquality limited under section 303(d) of the Federal Clean Water Act:

- Diamond Lake, for aquatic weeds or algae, dissolved oxygen, and $\mathrm{pH}$ (Diamond Lake was removed from the 303(d) list when the Total Maximum Daily Load (TMDL) was approved);

- Lemolo Lake, for aquatic weeds or algae;

- North Umpqua River (RM 77-78), for pH during summer;

- North Umpqua River (RM 0-68.9) for water temperature, year round (this segment was removed from 303[d] list when the TMDL was approved);

- North Umpqua River (RM 0-32) for water temperature during fish spawning periods (September 1-May 15); and

- North Umpqua River (RM 35.1-41.4) for water temperature during fish spawning periods (September 1-June 15).

\section{Study Background}

Diamond Lake (cover photograph 2), in the headwaters of the North Umpqua River in the Oregon Cascade Range (fig. 1), has had large blooms of blue-green algae for many years. In August 2001, a large bloom of Anabaena and subsequent detection of algal toxins, including anatoxin- $a$ and microcystin, closed the lake for all uses, including water contact, boating, and fishing (Jones and others, 2007; Eilers and others, 2011) (table 1). Anabaena blooms occurred in each of the following 4 years, decimating the economically important recreational fishery in the lake, and spurring a debate regarding the cause of the blooms. 


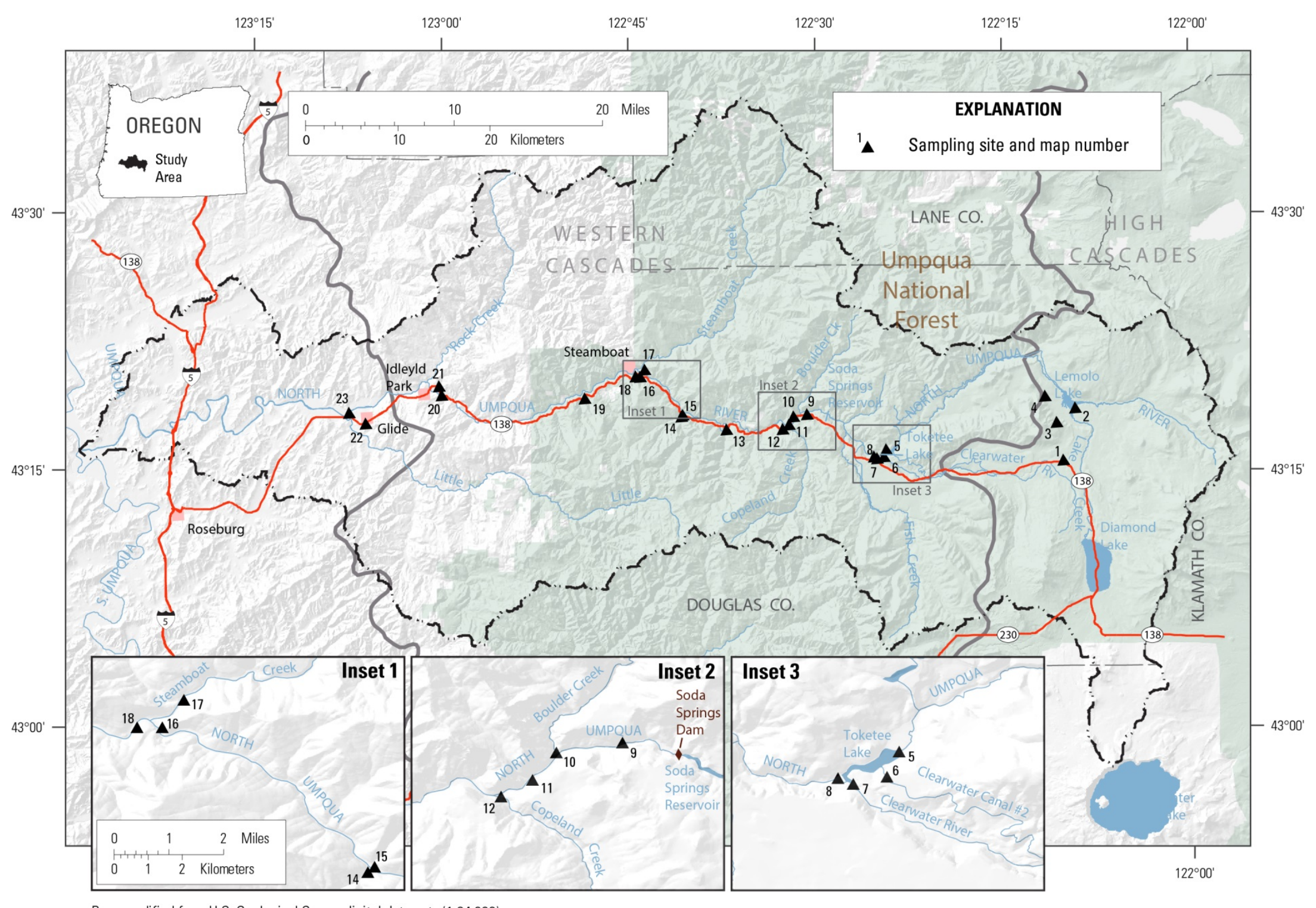

Base modified from U.S. Geological Survey digital data sets $(1: 24,000)$

Projection: UTM, Zone 11, North American Datum 1983

Figure 1. Map of the North Umpqua River basin showing the sampling-site locations. Sampling site numbers are listed in table 5. 
Table 1. History of blue-green algae blooms and associated human-health advisories and management actions in Diamond and Lemolo Lakes, North Umpqua River basin, Oregon, 2001-10.

\begin{tabular}{|c|c|c|c|c|c|}
\hline \multirow[b]{3}{*}{ Year } & \multicolumn{4}{|c|}{ Algae bloom alert level } & \multirow[b]{3}{*}{ All alerts lifted } \\
\hline & 1 & 2 & 3 & 4 & \\
\hline & $\begin{array}{c}\text { Begin } \\
\text { sampling }\end{array}$ & $\begin{array}{c}\text { Warning } \\
\text { date }\end{array}$ & $\begin{array}{c}\text { Water } \\
\text { contact } \\
\text { restriction }\end{array}$ & $\begin{array}{c}\text { All uses } \\
\text { (boat) } \\
\text { restricted }\end{array}$ & \\
\hline 2001 & 6 June $^{\mathrm{a}}$ & 10 Aug. & 10 Aug. & 17-25 Aug. & 27 Aug. \\
\hline 2002 & 6 May & 27 June & 23 July & -- & 7 Aug. \\
\hline 2003 & 28 May & 27 June $^{b}$ & $\begin{array}{l}1 \text { July- } \\
7 \text { Aug. }\end{array}$ & -- & 28 Aug. $^{\text {b }}$ \\
\hline 2004 & 10 May & 16 July & -- & -- & 27 Aug. $^{\text {b }}$ \\
\hline 2005 & 21 March & 24 June & -- & -- & \\
\hline 2006 & 16 May & -- & 5 Oct. & -- & 16 Nov. \\
\hline 2007 & 7 May & -- & -- & -- & \\
\hline 2008 & 27 May & -- & -- & -- & \\
\hline 2009 & 18 May & -- & -- & -- & -- \\
\hline 2010 & 7 June & -- & 15 July & -- & 3 Aug. \\
\hline 1993 & & labaena blc & om in Augu & & \\
\hline 1997 & & abaena blc & om in Augu & & \\
\hline 2006 & 11 July & -- & $\begin{array}{l}12 \text { July- } \\
2 \text { Aug.; } \\
26 \text { Sept. }\end{array}$ & -- & 13 October \\
\hline 2007 & 7 May & -- & 26 June & -- & 20 Aug. \\
\hline 2008 & 19 May & -- & $\begin{array}{l}10 \text { July- } \\
27 \text { Aug.; } \\
18 \text { Sept. }\end{array}$ & -- & 14 October \\
\hline 2009 & 27 April & -- & 23 July & -- & 18 Aug. \\
\hline 2010 & 15 March & -- & 1 July & -- & 2 Aug. \\
\hline
\end{tabular}

Tui chub (Gila bicolor), an invasive fish, probably introduced as live bait, was blamed for disrupting the food web and for enriching the lake with nutrients from their waste products (Eilers and others, 2007). The preferred alternative in the Final Environmental Impact Statement for the Diamond Lake restoration project (U.S. Forest Service, 2004) included the reduction of fish biomass in Diamond Lake. In September 2005, after years of planning, the Oregon Department of Fish and Wildlife (ODFW) initiated a drawdown of Diamond Lake through a drainage canal, ultimately dropping the lake level by $\sim 2.5 \mathrm{~m}$ (photograph 5), and in September 2006 treated the remaining water with the fish toxicant rotenone to eliminate the Tui chub. During the drawdown in 2006, ODFW harvested more than 30 metric tons of fish biomass from the lake to reduce the eventual load of carbon and nutrients from decomposing fish carcasses (Eilers and others, 2008; Eilers and Truemper, 2010). A timeline of significant events related to the rotenone application is shown in table 2.

Tui chub eradication involved a 10-month drawdown period, which was anticipated to release nutrientrich water from eutrophic Diamond Lake into Lake Creek and the downstream North Umpqua River. The Final Environmental Impact Statement required a comprehensive monitoring strategy, which included algae and water-quality monitoring in Diamond Lake, Lake Creek, and the Wild and Scenic Reach of 
Table 2. Timeline of data collection and events related to the rotenone application in Diamond Lake-

[Abbreviations: BSODA, North Umpqua River below Soda Springs Powerhouse; NUBWC, North Umpqua River at Wright Creek below Steamboat Creek; NURCR, North Umpqua River above Rock Creek at Idleyld; LAKE138, Lake Creek at Highway 138 below Diamond Lake; TOKLKI, North Umpqua River above Toketee Lake. For more information see Anderson and Carpenter (1998).]

\begin{tabular}{|c|c|c|c|}
\hline Year & Activity & Date & $\begin{array}{l}\text { Description of USGS } \\
\text { sampling }\end{array}$ \\
\hline$\overline{1993-94}$ & $\begin{array}{l}\text { USGS study of nutrients, major ions, field parameters, and bed } \\
\text { sediment in the Wild and Scenic Reach of the North Umpqua } \\
\text { River (NUBWC and NURCR) }\end{array}$ & various & -- \\
\hline 1994 & $\begin{array}{l}\text { USGS pilot study of nutrients and algae in the Wild and Scenic } \\
\text { Reach (NURCR) }\end{array}$ & July-Sept. & -- \\
\hline 1995 & USGS synoptic sampling for nutrients and algae & July $24-29,1995$ & Basinwide synoptic \\
\hline 2000 & $\begin{array}{l}\text { Continuous water-quality monitoring initiated at BSODA and } \\
\text { NURCR sites }\end{array}$ & Oct. 1,2000 & $\begin{array}{l}\text { Continuous water quality } \\
\text { monitors }\end{array}$ \\
\hline \multirow[t]{5}{*}{2005} & USGS synoptic sampling for nutrients and algae & July $25-29,2005$ & Basinwide synoptic \\
\hline & Diamond Lake pre-drawdown sampling & July 26,2005 & Monthly sites \\
\hline & Drawdown of Diamond Lake begins & Sept. 16, 2005 & -- \\
\hline & Flow begins in Lake Creek Canal & Nov. 23, 2005 & -- \\
\hline & $\begin{array}{l}\text { USGS Flow Record in Lake Creek Canal begins (USGS station } \\
\text { number 14312490) }\end{array}$ & Dec. 1,2005 & -- \\
\hline \multirow[t]{16}{*}{2006} & Flow in Lake Creek stops, reduced to canal flow only & Feb. 11, 2006 & -- \\
\hline & Flashboards in canal removed to accelerate drawdown rate & March 21, 2006 & -- \\
\hline & USGS Sampling resumes when snow conditions permit access & April 19, 2006 & Monthly sites \\
\hline & USGS Sampling in Lake Creek and downstream & May and June 2006 & Monthly sites \\
\hline & Drawdown of Diamond Lake completed & July 1, 2006 & -- \\
\hline & USGS Sampling in Lake Creek and downstream & July and Aug. 2006 & Monthly sites \\
\hline & USGS Sampling in Lake Creek and downstream & Aug. 14, 2006 & Three-site synoptic \\
\hline & $\begin{array}{l}\text { Continuous water-quality monitoring initiated at LAKE138 and } \\
\text { TOKLKI }\end{array}$ & Late Aug. & $\begin{array}{l}\text { Continuous water quality } \\
\text { monitors }\end{array}$ \\
\hline & USGS Sampling in Lake Creek and downstream & Aug. 30, 2006 & Monthly sites \\
\hline & All surface water discharge from Diamond Lake is stopped & Sept. 6, 2006 & -- \\
\hline & Rotenone application & Sept. 14-28, 2006 & -- \\
\hline & USGS Sampling in Lake Creek and downstream & Sept.-Oct. 2006 & Monthly sites \\
\hline & $\begin{array}{l}\text { Post-treatment flow from Diamond Lake resumes but is } \\
\text { intermittent }\left(<1-2 \mathrm{ft}^{3} / \mathrm{s}\right)\end{array}$ & Nov. 7-30, 2006 & -- \\
\hline & $\begin{array}{l}\text { Lake Creek Canal gage shows flow on a few days, generally } \\
<2 \mathrm{ft}^{3} / \mathrm{s} \text {, most days zero flow }\end{array}$ & Nov. 7-30, 2006 & -- \\
\hline & Lake Creek Canal flow increased to $10-20 \mathrm{ft}^{3} / \mathrm{s}$ & Nov. 30, 2006 & -- \\
\hline & USGS Sampling & Dec. 20,2006 & Monthly sites \\
\hline
\end{tabular}

Table continued on next page 
Table 2. Timeline of data collection and events related to the rotenone application in Diamond Lake-continued.

[Abbreviations: BSODA, North Umpqua River below Soda Springs Powerhouse; NUBWC, North Umpqua River at Wright Creek below Steamboat Creek; NURCR, North Umpqua River above Rock Creek at Idleyld; LAKE138, Lake Creek at Highway 138 below Diamond Lake; TOKLKI, North Umpqua River above Toketee Lake. For more information see Anderson and Carpenter (1998).]

\begin{tabular}{llcc}
\hline & \multicolumn{1}{c}{ Activity } & Date & $\begin{array}{c}\text { Description of USGS } \\
\text { sampling }\end{array}$ \\
\hline 2007 & USGS sampling resumes when snow conditions permit access & March 6, 2007 & Monthly sites \\
& Lake Creek flows resume & March 15, 2007 & -- \\
& Lake Creek canal fully closed & March 20, 2007 & -- \\
& USGS Sampling & March-June 2007 & Monthly sites \\
& Diamond Lake reaches full pool & July 12, 2007 & -- \\
& USGS Sampling & July 17, 2007 & Monthly sites \\
& USGS Sampling & Aug. 6-9, 2007 & Basinwide synoptic \\
& USGS Sampling & Sept. 12, 2007 & Monthly sites \\
& Diamond Lake reaches full pool, catchable and fingerling trout \\
stocked & June 1, 2007 & -- \\
\hline
\end{tabular}

the North Umpqua River downstream (fig. 1). During 2005-07, ODFW and the U.S. Forest Service (USFS) monitored Diamond Lake and USGS sampled Lake Creek and the downstream North Umpqua River (table 2). For comparison, USGS also sampled the North Umpqua River upstream of Lemolo Lake at the Inlet Campground (fig. 1, site 2), away from the effects of the Diamond Lake restoration.

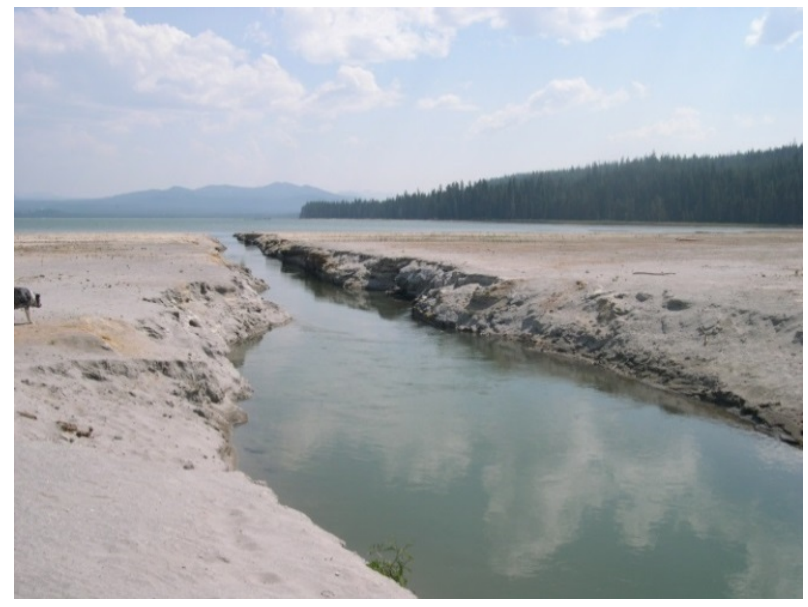

Photograph 5. Diamond Lake and outflow canal. (Photograph by Chauncey Anderson, U.S. Geological Survey, August 2, 2006.)

\section{Study Objectives, Approach, and Report Scope}

This report builds on a previous study Anderson and Carpenter (1998) that documented basinwide nutrient and algal conditions and provided background information for relicensing of the North Umpqua Hydroelectric Project. The study also began to address concerns from longtime anglers who had observed slippery rocks and reduced water clarity in the river that they associated with profuse growths of periphyton (Frank Moore, Idleyld Park, Oregon, oral commun., 1994), and documented compliance with State of Oregon $\mathrm{pH}$ and dissolved oxygen water-quality standards.

The current 2005-07 study provided a unique opportunity to examine whether river conditions had changed over the intervening decade since 1995. This report includes an analysis of water-quality and algal conditions in Lake Creek and the North Umpqua River collected in 2005-07 to evaluate conditions before, during, and after the September 2006 rotenone application. 
The objectives of the study were (1) to evaluate the effects of the Diamond Lake restoration project on water quality and algal conditions in the reach extending from Lake Creek downstream through the Wild and Scenic portion of the North Umpqua River (referred to hereafter as the "Diamond Lake Restoration Affected Reach"), and (2) compare 2005 and 1995 data for periphyton biomass and species composition, streamflow, water temperature, and nutrient concentrations changes prior to the restoration project.

The primary focus of this report is on waterquality and algal conditions in the Diamond Lake Restoration Affected Reach, which includes the Wild and Scenic Reach. We also describe changes in river management between 1995 and 2005 that complicated, to some degree, the evaluation of the downstream effects of the Diamond Lake restoration project. Data used were from weeklong synoptic surveys by USGS and others in July 1995 (Anderson and Carpenter, 1998), July 2005 (Tanner and others, 2006), and August 2007, and monthly samples collected during the restoration project from April 2006 through October 2007. The analysis included hourly data from (1) a streamgage in the Wild and Scenic Reach upstream of Copeland Creek, (2) continuous water-quality monitors in the North Umpqua River near Idleyld Park upstream of Rock Creek, and (3) temporary installations during 2006-07, including Lake Creek downstream from Diamond Lake (U.S. Geological Survey, 2013).

This analysis focuses on Lake Creek near the mouth, $10 \mathrm{mi}$ downstream from Diamond Lake, and the Wild and Scenic Reach downstream from the hydroelectric project (fig. 2). Data and findings by others involved in the Diamond Lake restoration project (Eilers and others 2007; 2008; Sytsma and others, 2007; Sytsma and Miller, 2008; Eilers and Truemper, 2010; Eilers and others, 2011; ODFW, http://www.dfw.state.or.us/fish/local_fisheries/di amond_lake/restoration-update.asp; U.S. Forest Service, http://www.fs.usda.gov/detail/umpqua/landmana gement/planning/?cid=stelprdb5336043) were considered for this analysis, and were used here for reference and qualitative purposes only.

It is beyond the scope of this report to evaluate the efficacy of the restoration (the rotenone application and subsequent lake management) relative to fisheries production or water quality in Diamond Lake itself. Observations suggest, however, that although there have been blue-green algae blooms in Diamond Lake since implementation of the restoration project, the blooms have been of shorter duration and less intense in recent years (Eilers and others, 2011; A1 Johnson, U.S. Forest Service, written commun., 2013).

\section{Study Area}

The North Umpqua River Basin, which covers approximately 1,350 square miles $\left(\mathrm{mi}^{2}\right)$, is part of the Umpqua River Basin in western Oregon (fig. 1). The area considered in this report extends from the headwaters to the town of Glide, an area of approximately 1,209 $\mathrm{mi}^{2}$. The report divides the study area into five sections (1) the upper North Umpqua River and Lake Creek, upstream of the hydroelectric project, (2) the hydroelectric project area, (3) the Wild and Scenic Reach downstream to Glide, (4) the Steamboat Creek Basin, and (5) the Little River watershed. For the purposes of this report, the Diamond Lake Restoration Affected Reach includes two sites on Lake Creek (LAKE138, downstream of Diamond Lake at Highway 138, and LAKEM, near the confluence with Lemolo Lake; fig. 1), and the main-stem sites downstream of Soda Springs Dam, continuing to the town of Glide. Some tributary sites were sampled to help determine nutrient loading and changes from 1995, but these sites otherwise were outside the influence of the restoration project; they were, therefore, excluded from analysis of the downstream effects of the restoration project. 


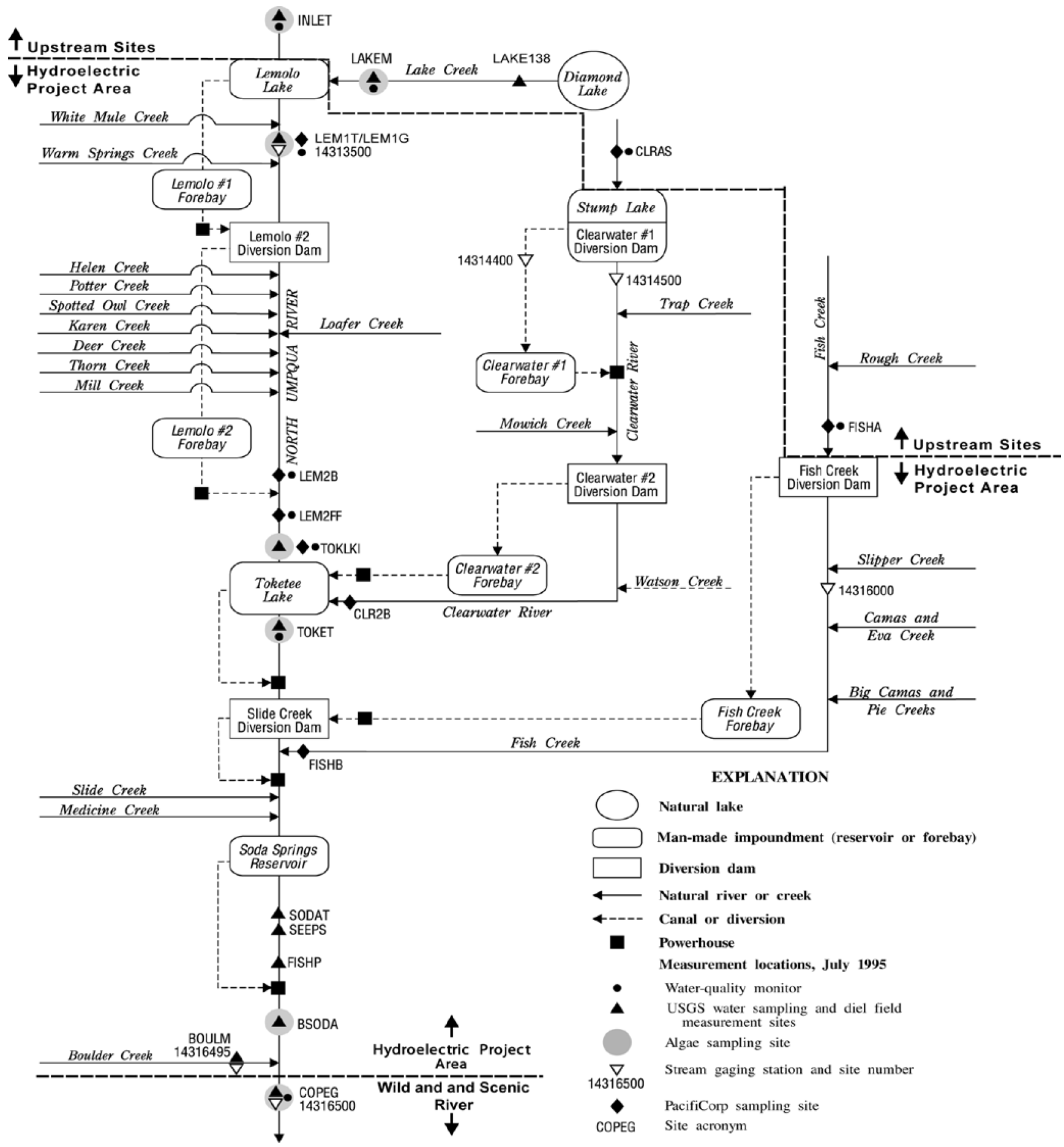

Figure 2. Schematic diagram of the upper North Umpqua River basin, Oregon, showing the complex routing of water through the Hydroelectric Project. Modified from Anderson and Carpenter (1998). Includes sites sampled in 1993-95 and 2005-07; not all sites were sampled in all years. 
The study area contains three geologic units: High Cascades, Western Cascades, and the Klamath Mountains. Together with elevation and weather, these geologic units exert contrasting influences on the hydrology and chemistry of the river and its tributaries (Anderson and Carpenter, 1998). High Cascade streams have relatively constant streamflow and carry a large proportion of discharge derived from groundwater. In contrast, Western Cascade streams receive much less groundwater and have greater drainage densities, and, as a result, have lower streamflow during summer (Tague and Grant, 2004). Groundwater discharging to High Cascades streams and springs generally contains relatively high concentrations of soluble reactive phosphorus (SRP) (0.07-0.08 mg/L, or higher), mostly as dissolved, inorganic, biologically available phosphorus (see for example Caldwell, 1998). In contrast, SRP concentrations in Western Cascades streams are often less than $0.01 \mathrm{mg} / \mathrm{L}$ (Carpenter, 2003). The Klamath Mountains, which constitute 11 percent of the basin, are present primarily at the lower altitudes, mostly in the Little River Basin.

\section{Wild and Scenic River Reach}

The Wild and Scenic River reach of the North Umpqua River extends $32 \mathrm{mi}$ from Boulder Creek to Rock Creek (fig. 1). The river is famous for its scenic qualities, whitewater boating, and fly fishing (particularly for wild steelhead). But, despite the beauty and the many recreational opportunities within the Wild and Scenic Reach, the river is no longer a natural, undisturbed system. The upstream hydroelectric project regulates flow in the river, and intensive timber harvesting and road building have influenced the watershed for many decades (PacifiCorp, 1998; U.S. Forest Service, 2001; Wallick and others, 2011).

Streamflow in the Wild and Scenic Reach averages 700-900 cubic feet per second $\left(\mathrm{ft}^{3} / \mathrm{s}\right)$ during the low-flow months of August and September, and is primarily derived from groundwater and outflow from Diamond and
Lemolo Lakes. Most of this flow is routed through a complex series of reservoirs, canals, penstocks, and turbines in the hydroelectric project (fig. 2) before it enters the Wild and Scenic Reach downstream of Soda Springs Reservoir at RM 69.7. Steamboat Creek, Rock Creek, and Little River are the three largest tributaries in the study area downstream of Boulder Creek; each tributary adds about 40-70 $\mathrm{ft}^{3} / \mathrm{s}$ (Moffatt and others, 1990; U.S. Geological Survey, 2014a). Several other small tributaries combined add about $50 \mathrm{ft}^{3} / \mathrm{s}$ during summer.

The Wild and Scenic Reach has interspersed riffles, glides, and runs, and topography consistent with a 0.5 percent gradient (U.S. Geological Survey, 1915). Most of the Wild and Scenic Reach has bedrock channels with ledges and deep pools, interspersed with boulders, cobbles, and gravel bars (PacifiCorp, 1995, volume 4). The size and stability of river substrate materials affects biological conditions throughout the food web, from algae to fish. North Umpqua River substrates are composed of relatively larger particles and are more stable than substrates in other area rivers (Wallick and others, 2011), partly because the upstream hydroelectric projects intercept sand and small gravel (PacifiCorp, 1995). This type of riverbed provides ideal conditions for periphyton, particularly those with attachment structures such as holdfasts (filamentous green algae) or mucilaginous attachment structures (stalked diatoms). Periphytic algae, rather than watercolumn types of algae (phytoplankton) dominate the primary production in the North Umpqua River and other high-velocity Cascade Range rivers because their attachment structures allow them to colonize substrates in high-velocity habitats.

Natural growths of periphytic algae in the North Umpqua River provide food for aquatic invertebrates, assimilate nutrients from the water column, and produce oxygen during the day (Anderson and Carpenter, 1998). Nuisance growths of periphyton and excessive rates of photosynthesis can, however, elevate $\mathrm{pH}$ to 
levels that may be unhealthy for aquatic life. Large changes in the periphyton community resulting from eutrophication may produce shifts in riverine food webs and affect secondary production of benthic invertebrates altering food availability for resident and anadromous fish populations.

\section{Hydroelectric Project}

The North Umpqua Hydroelectric Project was constructed between 1947 and 1956 and is currently operated by PacifiCorp under a Federal Energy Regulatory Commission (FERC) license reissued in 2003. The project (fig. 2) extends from the powerhouse at Soda Springs Reservoir (RM 69.7) upstream to Lemolo Lake. Lake Creek, which enters Lemolo Lake at RM 94.6, and the upper North Umpqua River, which enters Lemolo Lake at Inlet Campground, RM 94.8 (cover photograph 1; fig. 1, site 2), both originate outside the hydroelectric project. The project has eight facilities, and includes sections of the Clearwater River and Fish Creek. Each facility typically consists of a dam, canals and flumes, penstock, and powerhouse. There are $21.7 \mathrm{mi}$ of canals, $9.8 \mathrm{mi}$ of flumes, and $5.8 \mathrm{mi}$ of penstock and tunnels, for a total waterway length of $37.3 \mathrm{mi}$. Three reservoirs (Lemolo Lake, Toketee Lake, and Soda Springs Reservoir) and 4 forebays provide limited water storage. These impoundments range in size from about 15 to 450 surface acres (Anderson and Carpenter, 1998). Lemolo Lake is drawn down in winter for flow management purposes.

Hydroelectric operations have altered the natural hydrograph of most reaches within the hydroelectric project area significantly, and to a lesser extent downstream of the hydroelectric project. The new FERC license (Federal Energy Regulatory Commission, 2003) required increased in-stream flow within all project bypass reaches, which were implemented between 2005 and 2012 (table 3). Daily flow manipulation for power production (ramping) was ceased downstream of the project in 2003 and within bypass reaches in 2006 (except when required for project maintenance). These changes in flow regime, along with historically greater flow manipulations, may affect several water quality and biological processes such as nutrient uptake and recycling (spiraling) and food-web dynamics (Anderson and Carpenter, 1998).

Diversions for power generation alter the magnitude or timing of flows in many stream channels; flows in the bypassed natural stream channels (bypass reaches) tend to be lower and more stable than before regulation. During the original 1995 study, required minimum in-stream flows in the bypass reaches were between 5 and $25 \mathrm{ft}^{3} / \mathrm{s}$ (Anderson and Carpenter, 1998). During 2007 , the revised license required minimum midsummer flow of $80 \mathrm{ft}^{3} / \mathrm{s}$ in North Umpqua River bypass reaches downstream of Lemolo Lake (e.g. at LEM1T or LEM1G; cover photograph 4) except $275 \mathrm{ft}^{3} / \mathrm{s}$ in the Soda Springs bypass reach. In the tributaries, minimum required midsummer flows were $40 \mathrm{ft}^{3} / \mathrm{s}$ in the Clearwater River and $80 \mathrm{ft}^{3} / \mathrm{s}$ (or natural inflow, whichever was less) in Fish Creek. The North Umpqua River downstream of Lemolo Lake during the 2005 algal sampling was at a normal pre-2006 flow of about $38 \mathrm{ft}^{3} / \mathrm{s}$ because the specified increases had not yet gone into effect. 
Table 3. Minimum in-stream flows for bypass reaches of the North Umpqua River Hydroelectric Project required prior to and following issuance of the new Federal Energy Regulatory Commission license, settlement agreement, and 401 Water-Quality Certification.

[Data from Federal Energy Regulatory Commission (2003). Streamflow is reported in cubic feet per second. Required start dates: $2005=9-1-2005 ; 2006=12-312005$ (except Deer Creek, 10-18-2006); $2012=10-18-2012$.]

\begin{tabular}{|c|c|c|c|c|c|c|c|c|c|c|}
\hline \multirow[b]{3}{*}{ Month } & \multicolumn{10}{|c|}{ Reach } \\
\hline & \multirow{2}{*}{$\begin{array}{c}\text { Lemolo } 1 \\
1995 / \\
2006\end{array}$} & \multirow{2}{*}{$\begin{array}{c}\text { Lemolo } 2 \\
1995 / \\
2006\end{array}$} & \multirow{2}{*}{$\begin{array}{c}\text { Clearwater } \\
1 \text { and } 2 \\
1995 / \\
2006\end{array}$} & \multirow{2}{*}{$\begin{array}{c}\text { Toketee } \\
1995 / \\
2006\end{array}$} & \multicolumn{2}{|c|}{ Slide Creek } & \multicolumn{2}{|c|}{ Fish Creek $^{a}$} & \multirow{2}{*}{$\begin{array}{c}\begin{array}{c}\text { Soda } \\
\text { Springs }\end{array} \\
1995 / \\
2005\end{array}$} & \multirow{2}{*}{$\begin{array}{c}\begin{array}{c}\text { Deer } \\
\text { Creek }\end{array} \\
2006 \\
\end{array}$} \\
\hline & & & & & $\begin{array}{l}1995 / \\
2006\end{array}$ & 2012 & $\begin{array}{l}1995 / \\
2006\end{array}$ & 2012 & & \\
\hline January & $25 / 50$ & $25 / 50$ & $5 / 40$ & $25 / 60$ & $25 / 50$ & 240 & $10 / 50$ & 130 & $25 / 275$ & $1 /$ all \\
\hline February & $25 / 50$ & $25 / 50$ & $5 / 40$ & $25 / 60$ & $25 / 50$ & 240 & $10 / 50$ & 130 & $25 / 275$ & $1 /$ all \\
\hline March & $25 / 50$ & $25 / 50$ & $5 / 40$ & $25 / 60$ & $25 / 50$ & 240 & $10 / 50$ & 130 & $25 / 275$ & $1 /$ all \\
\hline April & $25 / 60$ & $25 / 60$ & $5 / 60$ & $25 / 60$ & $25 / 50$ & 240 & $20 / 50$ & 130 & $25 / 275$ & $1 /$ all \\
\hline May & $25 / 70$ & $25 / 70$ & $5 / 60$ & $25 / 60$ & $25 / 80$ & 240 & $20 / 50$ & 130 & $25 / 275$ & $1 /$ all \\
\hline June & $25 / 80$ & $25 / 70$ & $5 / 60$ & $25 / 80$ & $25 / 80$ & 240 & $20 / 80$ & 130 & $25 / 275$ & $1 /$ all \\
\hline July & $25 / 80$ & $25 / 80$ & $5 / 40$ & $25 / 80$ & $25 / 80$ & 240 & $20 / 80$ & 130 & $25 / 275$ & $1 /$ all \\
\hline August & $25 / 80$ & $25 / 80$ & $5 / 40$ & $25 / 80$ & $25 / 80$ & 240 & $20 / 80$ & 130 & $25 / 275$ & $1 /$ all \\
\hline September & $25 / 80$ & $25 / 80$ & $5 / 40$ & $25 / 80$ & $25 / 80$ & 240 & $10 / 80$ & 130 & $25 / 275$ & $1 /$ all \\
\hline October & $25 / 80$ & $25 / 80$ & $5 / 40$ & $25 / 80$ & $25 / 80$ & 240 & $10 / 80$ & 130 & $25 / 275$ & $1 /$ all \\
\hline November & $25 / 50$ & $25 / 50$ & $5 / 40$ & $25 / 60$ & $25 / 50$ & 240 & $10 / 50$ & 130 & $25 / 275$ & $1 /$ all \\
\hline December & $25 / 50$ & $25 / 50$ & $5 / 40$ & $25 / 60$ & $25 / 50$ & 240 & $10 / 50$ & 130 & $25 / 275$ & $1 /$ all \\
\hline
\end{tabular}

${ }^{\mathrm{a}}$ Typical winter and summer baseflow to the Fish Creek dam is $30-40 \mathrm{ft}^{3} / \mathrm{s}$, hence diversion is now ceased much of the year and actual streamflow is well below the specified minimum of $130 \mathrm{ft}^{3} / \mathrm{s}$.

\section{Diamond and Lemolo Lakes}

At more than 3,000 acres, Diamond Lake is the largest water body in the study area (fig. 1), and is heavily used for recreation. This shallow lake (mean depth $6.9 \mathrm{~m}$, residence time 1.6 years) is classified as "eutrophic-polluted" and commonly has spring and summer blooms of diatoms and blue-green algae (Oregon Department of Environmental Quality, 1996). The lake has been in this condition for much of its recreational history because of the large amount of internal nutrient loading (Salinas and Larson, 1995). Following blooms in the early 2000s, fisheries managers hypothesized that Tui chub, an invasive non-native fish sometimes used as bait, caused food-web changes that degraded water quality (Eilers and Truemper, 2010). Nutrient loading from natural and human sources, including phosphorus-rich groundwater, septic systems, and nitrogen fixation by bluegreen algae, contributed to nuisance blooms that continued to occur despite the diversion in 1971 of sewage from public recreation and fish cleaning facilities (Eilers and others, 2003; U.S. Forest Service, 2004).

Lake Creek is a 10-mile-long waterway that connects Diamond and Lemolo Lakes (fig. 1). The creek gains additional flow from groundwater and tributaries, and contributes approximately $30-50 \mathrm{ft}^{3} / \mathrm{s}$ of flow to Lemolo Lake during the summer; the annual mean flow is about $57 \mathrm{ft}^{3} / \mathrm{s}$ (Moffatt and others, 1990).

Lemolo Lake, at 400 acres, is the secondlargest water body and the largest man-made impoundment in the study area. The upper North Umpqua River usually discharges more than 290 
$\mathrm{ft}^{3} / \mathrm{s}$ to Lemolo Lake during summer (PacifiCorp, 1995, volume 3). Like Diamond Lake, Lemolo Lake is a popular destination for boating, fishing, swimming, and camping. Also like Diamond Lake, Lemolo Lake sometimes has potentially toxic blooms of Anabaena in summer (Oregon Department of Human Services, 2010; table 1; photograph 6). Lemolo Lake is considered mesotrophic (moderately enriched with nutrients) based on chlorophyll-a (Chl-a) concentrations and $\mathrm{pH}$; occasional blue-green algae blooms indicate a more eutrophic state. Blooms have occurred intermittently for nearly 20 years, but in 2006 the increased magnitude resulted in advisories for five consecutive summers (table 1).

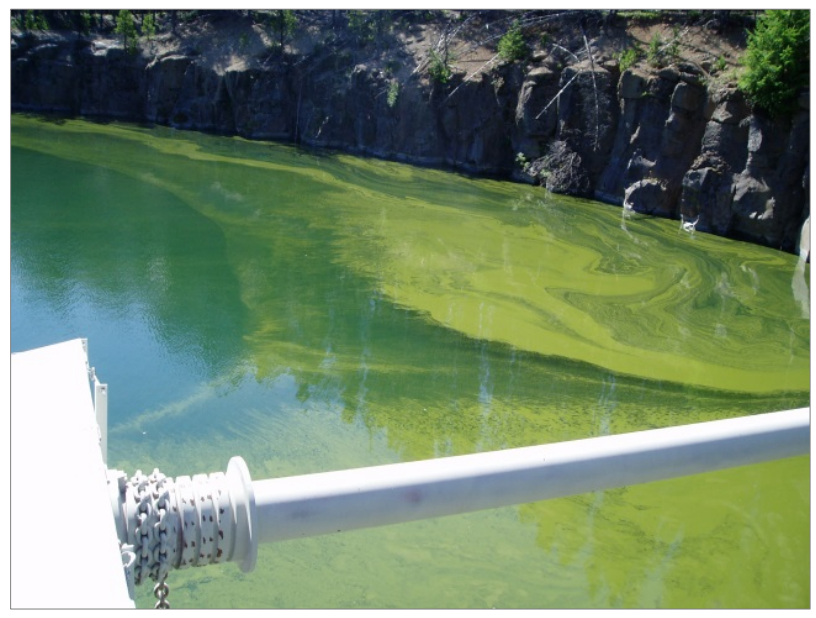

Photograph 6. Anabaena bloom in Lemolo Lake. (Photograph by Kurt Carpenter, U.S. Geological Survey, July 2007.)

During some algal blooms, Lemolo Lake exceeds the State of Oregon water-quality standard of $8.5 \mathrm{pH}$ units (PacifiCorp, 1995; U.S. Geological Survey, unpublished data, 1997). The reservoir is on the Oregon Department of Environmental Quality (2010) 303(d) list for $\mathrm{pH}$ and algae). Maximum summertime values for dissolved oxygen (DO) and $\mathrm{pH}$ often occur in the reservoir thermocline (PacifiCorp, 1995; Bonoff and others, 1996), a situation indicative of algal production. Lemolo Lake is thermally stratified during summer, but inputs of cold oxygenated water from the upper North Umpqua River and the relatively short residence times likely prevent low-DO conditions from developing in the hypolimnion (Rinella, 1979; Johnson and others, 1985; PacifiCorp, 1995).

\section{Methods}

\section{Data Collection}

Sampling began in 2005 at each site prior to the drawdown of Diamond Lake, and continued through the drawdown period, the rotenone application in September 2006, and afterward as outflows from the lake into Lake Creek resumed (tables 2 and 4). Data collection culminated in a post-restoration synoptic sampling during summer 2007. Most 2005-07 sites (table 5) were also sampled in 1995 (Anderson and Carpenter, 1998), including Lake Creek at the mouth (LAKEM) and selected sites in the hydroelectric project area, Wild and Scenic Reach, and tributaries. LEM1T and LEM1G are near each other and are used interchangeably (denoted LEM1T/G) in this report. At each site, we measured water-quality parameters (water temperature, specific conductance, $\mathrm{DO}$, and $\mathrm{pH}$ ) and collected samples for nutrients and periphyton analyses. To understand the patterns and magnitudes of primary production in the river, we measured DO and $\mathrm{pH}$ in the early morning and mid-afternoon at most main-stem and selected tributary sites. Samples were analyzed for particulate and dissolved forms of organic carbon, phosphorus, and nitrogen because their export from Diamond Lake was expected to increase during the restoration project (U.S. Forest Service, 2004). The 1995 study showed that organically derived nutrients are an important factor in the periphyton community structure in the North Umpqua River. These nutrients stimulate the growth of nitrogen-heterotrophic diatoms and associated microbial communities that can use organically derived nitrogen in addition to the biologically available dissolved inorganic forms (Anderson and Carpenter, 1998). 
Standard USGS methods were used for sampling and analysis except where noted. Data collection methods were the same as those used by Anderson and Carpenter (1998) and Tanner and others (2006). Douglas County, ODFW, ODEQ, PacifiCorp, and the USFS provided assistance during the field surveys.

Table 4. Field parameter data collection and long term continuous monitor deployment periods for sites in the North Umpqua River basin, 2005-07.

[Field parameters included water temperature, specific conductance, $\mathrm{pH}$, dissolved oxygen, and, at some sites, turbidity. Site codes are defined in table 5. Data from long-term deployments are available at the USGS Oregon Water Science Center's Data Grapher website, http://or.water.usgs.gov/grapher/.]

\begin{tabular}{|c|c|c|c|c|}
\hline Site Code & $\begin{array}{c}\text { Temporary } \\
\text { deployment } \\
\text { (18-24 hours) }\end{array}$ & $\begin{array}{l}\text { Long-term } \\
\text { deployment } \\
\text { (>5 days) }\end{array}$ & Deployment date & Completion date \\
\hline \multicolumn{5}{|c|}{2005 Synoptic survey } \\
\hline LAKEM & $\mathrm{X}$ & & July 25,2005 & July 26, 2005 \\
\hline TOKET & $\mathrm{X}$ & & July 24,2005 & July 25,2005 \\
\hline BSODA & & $\mathrm{X}$ & October 1, 2000 & $--^{b}$ \\
\hline COPEG & $\mathrm{X}$ & & July $27,2005^{\text {a }}$ & July $29,2005^{a}$ \\
\hline STEAM & $\mathrm{X}$ & & July 28, 2005 & July 29, 2005 \\
\hline NUSTM & $\mathrm{X}$ & & July 28,2005 & July 30, 2005 \\
\hline NURCR & & $\mathrm{X}$ & October 1,2000 & $--^{b}$ \\
\hline \multicolumn{5}{|c|}{ 2006-07 Monthly water-quality sampling and continuous monitors } \\
\hline LAKE138 & & $\mathrm{X}$ & September 1, 2006 & November 6, 2007 \\
\hline TOKLKI & & $\mathrm{X}$ & August 31, 2006 & November 6, 2007 \\
\hline BSODA & & $\mathrm{X}$ & October 1,2000 & $--b^{b}$ \\
\hline COPEG & & $\mathrm{X}$ & September 1, 2006 & November 6, 2007 \\
\hline NURCR & & $\mathrm{X}$ & October 1, 2000 & $--^{\mathrm{b}}$ \\
\hline \multicolumn{5}{|c|}{2007 Synoptic survey } \\
\hline LAKE138 & & $\mathrm{X}$ & September 1, 2006 & November 6, 2007 \\
\hline INLET & $\mathrm{X}$ & & August 6, 2007 & August 7, 2007 \\
\hline TOKLKI & & $\mathrm{X}$ & August 31, 2006 & November 6, 2007 \\
\hline TOKET & $\mathrm{X}$ & & August 6, 2007 & August 7, 2007 \\
\hline BSODA & & $\mathrm{X}$ & October 1, 2000 & September $30,2007^{b}$ \\
\hline COPEG & & $\mathrm{X}$ & September 1, 2006 & November 6, 2007 \\
\hline CALFM & $\mathrm{X}$ & & August 7, 2007 & August 8, 2007 \\
\hline NUAPC & $\mathrm{X}$ & & August 7, 2007 & August 8, 2007 \\
\hline BOULDM & $\mathrm{X}$ & & August 7, 2007 & August 8, 2007 \\
\hline STEAM & $\mathrm{X}$ & & August 8, 2007 & August 9, 2007 \\
\hline NUSTM & $\mathrm{X}$ & & August 8, 2007 & August 9, 2007 \\
\hline NURCR & & $\mathrm{X}$ & October 1, 2000 & September $30,2007^{b}$ \\
\hline LITGL & $\mathrm{X}$ & & August 8, 2007 & August 9, 2007 \\
\hline
\end{tabular}

\footnotetext{
${ }^{\mathrm{a}}$ Instantaneous measurements collected at approximate minimum and maximum diel conditions.

${ }^{b}$ Monitor is still in operation as of the publication date for this report.
} 


\section{Water-Quality Samples and Field Measurements}

Water samples were collected according to standard USGS protocols (Wilde and others, 1999). During 2005-07, the ODEQ laboratory provided analysis, according to ODEQ protocols (Oregon Department of Environmental Quality, 1998), which are generally consistent with USGS protocols. Tanner and others (2006) documented ODEQ analytical methods and reporting limits (appendix table A1).

Water samples were composited from multiple vertical collections through the full cross section at most all sites using the EqualWidth-Increment (EWI) method of Edwards and Glysson (1999) adapted for low-concentration part-per-billion sampling (Wilde and others, 1999). At high flows, as in 2006, safety considerations precluded cross-sectional wading at the North Umpqua River above Copeland Creek (photograph 7); at Rock Creek a grab sample was collected from shore. Samples for dissolved constituents were filtered in the field using Gelman capsule filters with a pore size of 0.45 micrometer $(\mu \mathrm{m})$. Samples for nutrients and Chl- $a$ were preserved according to ODEQ requirements (Oregon Department of Environmental Quality, 2006). In this report, nitrite-plus-nitrate concentrations are referred to as nitrate $\left(\mathrm{NO}_{3}\right)$ and ammonia concentrations as ammonium $\left(\mathrm{NH}_{4}\right)$ because these are the predominant forms of dissolved inorganic nitrogen (DIN) in North Umpqua River Basin surface waters (Anderson and Carpenter, 1998). During periods of high $\mathrm{pH}$, un-ionized ammonia $\left(\mathrm{NH}_{3}\right)$ may be present, but total ammonia concentrations were relatively low and ammonia toxicity is unlikely in this system.

One notable difference in nutrient processing between surveys in 1995 and 20052007 warrants discussion. In 1995, samples for DIN were filtered to yield dissolved $\mathrm{NO}_{3}$ and $\mathrm{NH}_{4}$ concentrations (Anderson and Carpenter, 1998), whereas in 2005-07, $\mathrm{NO}_{3}$ and $\mathrm{NH}_{4}$ were taken from unfiltered samples that were preserved with sulfuric acid, in accordance with USEPA methods (appendix table A1).

Nonetheless, quality assurance samples showed no detectable difference between these methods. Furthermore, analysts at USGS (U.S. Geological Survey 1992) and ODEQ laboratories (Zach Mandera, Oregon Department of Environmental Quality, oral commun., September 2014) support the inherent assumption that $\mathrm{NO}_{3}$ and $\mathrm{NH}_{4}$ are overwhelmingly present in the dissolved phase and are therefore unaffected by the difference in methods. For the purposes of this report data from the two laboratories are used interchangeably. Where differences in $\mathrm{NO}_{3}$ or $\mathrm{NH}_{4}$ concentrations are noted between surveys involving the different analytical methods, results were carefully examined to verify that the reported changes are robust and independent of any methodological changes.

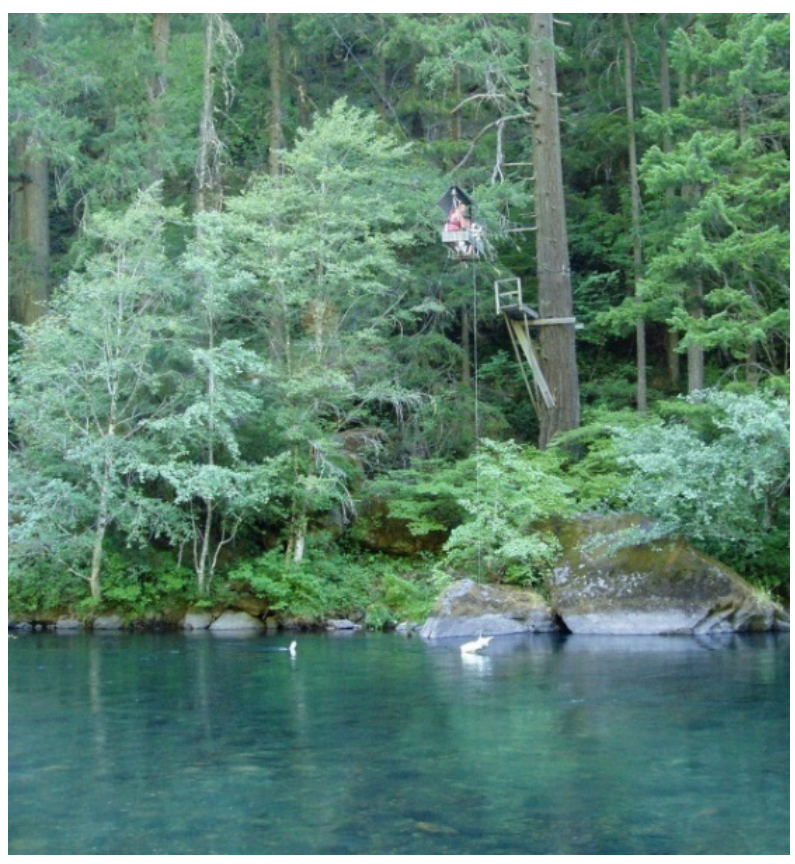

Photograph 7. Water sampling from the cableway across the North Umpqua River upstream of Copeland Creek (photograph taken by Kurt Carpenter, U.S. Geological Survey, July 26, 2005) 
Table 5. Water quality and algae sampling sites in the North Umpqua River basin, Oregon, 1995-2007

[Refer to figure 1 for map locations. Field values include stream temperature, specific conductance, alkalinity, and morning and afternoon levels of water temperature, $\mathrm{pH}$, dissolved oxygen, and variously, turbidity and chlorophyll- $a$. Water samples were analyzed for nutrients, alkalinity, chloride, and silica. Periphyton samples were analyzed for chlorophyll-a, biomass, species identification, and biovolume. Abbreviations: no., number; RM, river mile; USGS, U.S. Geological Survey; X, measurement made/sample collected; M, measured; G, gaged; E, estimated; --, not applicable or not determined]

\begin{tabular}{|c|c|c|c|c|c|c|c|c|c|c|c|c|c|}
\hline \multirow{2}{*}{$\begin{array}{c}\text { Map } \\
\text { index } \\
\text { no. } \\
\end{array}$} & \multirow{2}{*}{$\begin{array}{c}\begin{array}{c}\text { Main- } \\
\text { stem } \\
\text { river mile }\end{array} \\
\end{array}$} & \multirow{2}{*}{$\begin{array}{l}\text { Tributary } \\
\text { river } \\
\text { mile } \\
\end{array}$} & \multirow[b]{2}{*}{ Site code } & \multirow[b]{2}{*}{ Sampling site } & \multirow[b]{2}{*}{ USGS site number } & \multirow{2}{*}{$\begin{array}{c}\text { Field } \\
\text { parameters }\end{array}$} & \multirow{2}{*}{$\begin{array}{c}\text { Water } \\
\text { samples }\end{array}$} & \multirow{2}{*}{$\begin{array}{c}\text { Stream- } \\
\text { flow }\end{array}$} & \multirow{2}{*}{$\begin{array}{l}\text { Periphyton } \\
\text { algae } \\
\end{array}$} & \multicolumn{4}{|c|}{ Year sampled } \\
\hline & & & & & & & & & & 1995 & 2005 & 2006 & 2007 \\
\hline 2 & 94.8 & -- & INLET & $\begin{array}{l}\text { North Umpqua River above } \\
\text { Lemolo Lake near Inlet } \\
\text { Campground }\end{array}$ & 431839122091300 & X & $\mathrm{X}$ & M & $\mathrm{X}$ & $\mathrm{X}$ & $X$ & -- & $X$ \\
\hline 1 & 94.8 & 4.4 & LAKE138 & $\begin{array}{l}\text { Lake Creek at Highway } 138 \\
\text { below Diamond Lake }\end{array}$ & 14312600 & $\mathrm{X}$ & $\mathrm{X}$ & M & -- & -- & -- & -- & $\mathrm{X}$ \\
\hline 3 & 93.8 & 1 & LAKEM & $\begin{array}{l}\text { Lake Creek above Lemolo } \\
\text { Lake }\end{array}$ & 431750122104500 & $\mathrm{X}$ & $\mathrm{X}$ & M & $\mathrm{X}$ & $\mathrm{X}$ & $\mathrm{X}$ & $\mathrm{X}$ & $\mathrm{X}$ \\
\hline-- & 92.9 & -- & LEM1T & $\begin{array}{l}\text { Top of Lemolo } 1 \text { bypass } \\
\text { reach }\end{array}$ & 431924122111700 & $\mathrm{X}$ & $\mathrm{X}$ & -- & -- & $\mathrm{X}$ & -- & -- & -- \\
\hline 4 & 92.6 & -- & LEM1G & $\begin{array}{l}\text { North Umpqua River below } \\
\text { Lemolo Lake (gaged as } \\
\text { 14313500) }\end{array}$ & 14313500 & $\mathrm{X}$ & $X$ & G & $\mathrm{X}$ & $\mathrm{X}$ & $X$ & -- & $X$ \\
\hline-- & 77.3 & -- & LEM2B & $\begin{array}{l}\text { North Umpqua River above } \\
\text { Lemolo No. } 2 \text { Powerhouse }\end{array}$ & -- & $\mathrm{X}$ & $\mathrm{X}$ & -- & -- & $\mathrm{X}$ & -- & -- & -- \\
\hline-- & 77.1 & -- & LEM2FF & $\begin{array}{l}\text { North Umpqua River below } \\
\text { Lemolo No. } 2 \text { Powerhouse }\end{array}$ & -- & $\mathrm{X}$ & $\mathrm{X}$ & -- & -- & $\mathrm{X}$ & -- & -- & -- \\
\hline 5 & 77.1 & -- & TOKLKI & $\begin{array}{l}\text { North Umpqua River above } \\
\text { Toketee Lake }\end{array}$ & 14314010 & $\mathrm{X}$ & $\mathrm{X}$ & -- & $\mathrm{X}$ & & $\mathrm{X}$ & $\mathrm{X}$ & $\mathrm{X}$ \\
\hline 7 & 75.4 & 11.1 & CLRAS & $\begin{array}{l}\text { Clearwater River above } \\
\text { Stump Lake }\end{array}$ & -- & $\mathrm{X}$ & $\mathrm{X}$ & -- & -- & $\mathrm{X}$ & -- & -- & -- \\
\hline 6 & 75.4 & 0.0 & CLR2B & $\begin{array}{l}\text { Clearwater Canal \# } 2 \text { Bypass } \\
\text { Reach }\end{array}$ & -- & $X$ & $X$ & G & -- & $\mathrm{X}$ & -- & -- & - \\
\hline 8 & 75.4 & -- & TOKET & $\begin{array}{l}\text { North Umpqua River below } \\
\text { Toketee Lake }\end{array}$ & 431552122252100 & $\mathrm{X}$ & $\mathrm{X}$ & M & $\mathrm{X}$ & $\mathrm{X}$ & $\mathrm{X}$ & -- & X \\
\hline
\end{tabular}


Table 5. Water quality and algae sampling sites in the North Umpqua River basin, Oregon, 1995-2007-continued

[Refer to figure 1 for map locations. Field values include stream temperature, specific conductance, alkalinity, and morning and afternoon levels of water temperature, $\mathrm{pH}$, dissolved oxygen, and variously, turbidity and chlorophyll- $a$. Water samples were analyzed for nutrients, alkalinity, chloride, and silica. Periphyton samples were analyzed for chlorophyll- $a$, biomass, species identification, and biovolume. Abbreviations: no., number; RM, river mile; USGS, U.S. Geological Survey; X, measurement made/sample collected; M, measured; G, gaged; E, estimated; --, not applicable or not determined]

\begin{tabular}{|c|c|c|c|c|c|c|c|c|c|c|c|c|c|}
\hline \multirow{2}{*}{$\begin{array}{c}\text { Map } \\
\text { index } \\
\text { no. }\end{array}$} & \multirow{2}{*}{$\begin{array}{c}\text { Main- } \\
\text { stem } \\
\text { river mile }\end{array}$} & \multirow{2}{*}{$\begin{array}{c}\text { Tributary } \\
\text { river } \\
\text { mile }\end{array}$} & \multirow[b]{2}{*}{ Site code } & \multirow[b]{2}{*}{ Sampling site } & \multirow[b]{2}{*}{ USGS site number } & \multirow{2}{*}{$\begin{array}{c}\text { Field } \\
\text { parameters }\end{array}$} & \multirow{2}{*}{$\begin{array}{c}\text { Water } \\
\text { samples }\end{array}$} & \multirow{2}{*}{$\begin{array}{l}\text { Stream- } \\
\text { flow }\end{array}$} & \multirow{2}{*}{$\begin{array}{l}\text { Periphyton } \\
\text { algae }\end{array}$} & \multicolumn{4}{|c|}{ Year sampled } \\
\hline & & & & & & & & & & 1995 & 2005 & 2006 & 2007 \\
\hline-- & 71.8 & 6.6 & FISHA & $\begin{array}{l}\text { Fish Creek above Fish Creek } \\
\text { Diversion }\end{array}$ & -- & $X$ & $X$ & -- & -- & $X$ & -- & -- & -- \\
\hline-- & 71.8 & 0.0 & FISHB & Fish Creek at mouth & -- & $\mathrm{X}$ & $\mathrm{X}$ & -- & -- & $\mathrm{X}$ & -- & -- & -- \\
\hline-- & 69.6 & -- & FISHP & $\begin{array}{l}\text { Fish pond below Soda } \\
\text { Springs Reservoir }\end{array}$ & -- & $X$ & $\mathrm{X}$ & $\mathrm{E}$ & -- & $X$ & -- & -- & -- \\
\hline-- & 69.7 & -- & SODAT & $\begin{array}{l}\text { North Umpqua River below } \\
\text { Soda Springs }\end{array}$ & 431814122295100 & $X$ & $\mathrm{X}$ & M & -- & $X$ & -- & -- & -- \\
\hline-- & 69.7 & -- & SEEPS & $\begin{array}{l}\text { Seep at left bank below Soda } \\
\text { Springs Reservoir }\end{array}$ & 431813122295100 & $\mathrm{X}$ & $\mathrm{X}$ & $\mathrm{E}$ & -- & $X$ & -- & -- & -- \\
\hline 9 & 69.0 & -- & BSODA & $\begin{array}{l}\text { North Umpqua River below } \\
\text { Soda Springs Powerhouse }\end{array}$ & 431824122300600 & $X$ & $X$ & -- & $X$ & $X$ & $X$ & -- & $X$ \\
\hline 10 & 67.9 & 0.0 & BOULM & Boulder Creek near mouth & 14316495 & $X$ & $X$ & $\mathrm{G}$ & -- & $\mathrm{X}$ & $X$ & -- & $\mathrm{X}$ \\
\hline 11 & 67.2 & -- & COPEG & $\begin{array}{l}\text { North Umpqua River above } \\
\text { Copeland Creek }\end{array}$ & 14316500 & $\mathrm{X}$ & $X$ & G & $\mathrm{X}$ & $X$ & $\mathrm{X}$ & -- & $\mathrm{X}$ \\
\hline 12 & 66.6 & 0.0 & COPEM & Copeland Creek near mouth & 431730122323800 & $X$ & $\mathrm{X}$ & M & -- & $\mathrm{X}$ & $\mathrm{X}$ & -- & $\mathrm{X}$ \\
\hline 13 & 61.8 & 0.0 & CALFM & Calf Creek near mouth & 431728122370800 & $\mathrm{X}$ & $\mathrm{X}$ & M & -- & $\mathrm{X}$ & $\mathrm{X}$ & -- & $\mathrm{X}$ \\
\hline 14 & 57.7 & 0.0 & PANTM & Panther Creek near mouth & 431815122404400 & $\mathrm{X}$ & $\mathrm{X}$ & M & -- & $\mathrm{X}$ & $\mathrm{X}$ & -- & $\mathrm{X}$ \\
\hline 15 & 57.5 & -- & NUAPC & $\begin{array}{l}\text { North Umpqua River at } \\
\text { Apple Creek }\end{array}$ & 431820122403700 & $\mathrm{X}$ & $\mathrm{X}$ & M & -- & $\mathrm{X}$ & $\mathrm{X}$ & -- & $\mathrm{X}$ \\
\hline 16 & 53.2 & -- & MOTTB & $\begin{array}{l}\text { North Umpqua River at Mott } \\
\text { Bridge }\end{array}$ & 432034122440100 & $\mathrm{X}$ & $\mathrm{X}$ & M & -- & $\mathrm{X}$ & $\mathrm{X}$ & -- & $\mathrm{X}$ \\
\hline-- & 53.0 & 11.9 & STEABB & $\begin{array}{l}\text { Steamboat Creek above Big } \\
\text { Bend Creek }\end{array}$ & 432523122360300 & $\mathrm{X}$ & $X$ & M & $X$ & $X$ & -- & -- & -- \\
\hline-- & 53.0 & $0^{\mathrm{a}}$ & CANTM & Canton Creek near mouth & 432100122434300 & $\mathrm{X}$ & $\mathrm{X}$ & $\mathrm{G}$ & -- & $\mathrm{X}$ & -- & -- & -- \\
\hline
\end{tabular}

Table continued on next page 
Table 5. Water quality and algae sampling sites in the North Umpqua River basin, Oregon, 1995-2007-continued

[Refer to figure 1 for map locations. Field values include stream temperature, specific conductance, alkalinity, and morning and afternoon levels of water temperature, $\mathrm{pH}$, dissolved oxygen, and variously, turbidity and chlorophyll- $a$. Water samples were analyzed for nutrients, alkalinity, chloride, and silica. Periphyton samples were analyzed for chlorophyll- $a$, biomass, species identification, and biovolume. Abbreviations: no., number; RM, river mile; USGS, U.S. Geological Survey; X, measurement made/sample collected; M, measured; G, gaged; E, estimated; --, not applicable or not determined]

\begin{tabular}{|c|c|c|c|c|c|c|c|c|c|c|c|c|c|}
\hline \multirow{2}{*}{$\begin{array}{c}\text { Map } \\
\text { index } \\
\text { no. }\end{array}$} & \multirow{2}{*}{$\begin{array}{l}\text { Main- } \\
\text { stem } \\
\text { river mile }\end{array}$} & \multirow{2}{*}{$\begin{array}{l}\text { Tributary } \\
\text { river } \\
\text { mile }\end{array}$} & \multirow[b]{2}{*}{ Site code } & \multirow[b]{2}{*}{ Sampling site } & \multirow[b]{2}{*}{ USGS site number } & \multirow{2}{*}{$\begin{array}{l}\text { Field } \\
\text { parameters }\end{array}$} & \multirow{2}{*}{$\begin{array}{l}\text { Water } \\
\text { samples }\end{array}$} & \multirow{2}{*}{$\begin{array}{l}\text { Stream- } \\
\text { flow }\end{array}$} & \multirow{2}{*}{$\begin{array}{c}\text { Periphyton } \\
\text { algae }\end{array}$} & \multicolumn{4}{|c|}{ Year sampled } \\
\hline & & & & & & & & & & 1995 & 2005 & 2006 & 2007 \\
\hline 17 & 53.0 & 0.5 & STEAM & Steamboat Creek at gage & 14316700 & $\mathrm{X}$ & $\mathrm{X}$ & $\mathrm{G}$ & $\mathrm{X}$ & $\mathrm{X}$ & $\mathrm{X}$ & -- & $\mathrm{X}$ \\
\hline 18 & 52.5 & -- & NUSTM & $\begin{array}{l}\text { North Umpqua River near } \\
\text { Steamboat }\end{array}$ & 432034122442500 & $\mathrm{X}$ & $\mathrm{X}$ & -- & $\mathrm{X}$ & $\mathrm{X}$ & $\mathrm{X}$ & -- & $\mathrm{X}$ \\
\hline 19 & 48.2 & -- & NUBWC & $\begin{array}{l}\text { North Umpqua River below } \\
\text { Wright Creek }\end{array}$ & 14316800 & $\mathrm{X}$ & $\mathrm{X}$ & $\mathrm{G}$ & $x^{b}$ & -- & $\mathrm{X}$ & -- & $\mathrm{X}$ \\
\hline-- & 48.1 & 0.0 & WRCRM & Wright Creek near mouth & 431912122482700 & $\mathrm{X}$ & -- & M & -- & $\mathrm{X}$ & & -- & $\mathrm{X}$ \\
\hline-- & 42.1 & 0.0 & SUSNM & Susan Creek near mouth & 14317100 & $\mathrm{X}$ & -- & $\mathrm{E}$ & -- & $\mathrm{X}$ & -- & -- & -- \\
\hline-- & 36.2 & -- & $\mathrm{NURCR}^{\mathrm{e}}$ & $\begin{array}{l}\text { North Umpqua River above } \\
\text { Rock Creek }\end{array}$ & 14317500 & $\mathrm{X}$ & $X$ & M & $\mathrm{X}$ & $X$ & $\mathrm{X}$ & $X$ & $\mathrm{X}$ \\
\hline 20 & 35.8 & -- & $\mathrm{NURCR}^{\mathrm{e}}$ & $\begin{array}{l}\text { Water-quality monitor on } \\
\text { North Umpqua River near } \\
\text { Idleyld Park }\end{array}$ & 14317500 & $\mathrm{X}$ & -- & -- & -- & $\mathrm{X}$ & $\mathrm{X}$ & $\mathrm{X}$ & $\mathrm{X}$ \\
\hline 21 & 35.7 & 0.0 & ROCKM & Rock Creek near mouth & 432000123000700 & $\mathrm{X}$ & $X$ & M & -- & $\mathrm{X}$ & $\mathrm{X}$ & -- & -- \\
\hline-- & 29.1 & 20.7 & LTBLK & $\begin{array}{l}\text { Little River above Black } \\
\text { Creek }\end{array}$ & 431221122492100 & $\mathrm{X}$ & -- & M & -- & $\mathrm{X}$ & -- & -- & -- \\
\hline-- & 29.1 & 13 & LTWLF & $\begin{array}{l}\text { Little River at Wolf Creek } \\
\text { Job Center }\end{array}$ & 431453122553400 & $X$ & -- & $\mathrm{M}$ & -- & $X$ & -- & -- & -- \\
\hline 22 & 29.1 & 0.04 & LITGL & Little River near Glide & 431750123060000 & $\mathrm{X}$ & $X$ & M & $X$ & $X$ & $\mathrm{X}$ & -- & $X$ \\
\hline 23 & 27.8 & -- & NUGLD & $\begin{array}{l}\text { North Umpqua River near } \\
\text { Glide }\end{array}$ & $431827123072200^{d}$ & $\mathrm{X}$ & $\mathrm{X}$ & M & -- & $\mathrm{X}$ & $\mathrm{X}$ & -- & $\mathrm{X}$ \\
\hline
\end{tabular}

${ }^{a}$ Canton Creek enters Steamboat Creek at river mile 0.51 .

${ }^{\mathrm{b}}$ Periphyton samples were not collected at this site in 1995.

${ }^{\mathrm{c}}$ Samples were analyzed for filtered trace elements, suspended sediment, and filtered organic carbon.

${ }^{\mathrm{d}}$ This location was gaged from 1916-18 and 1928-38 as site number 14318500 .

${ }^{\mathrm{e}}$ Site were very close together and considered one site for purposes of the study. 


\section{Continuous Water-Quality Monitors}

Data for water-quality field measurements and continuous monitors were collected and processed according to USGS protocols (Wagner and others, 2006), and were published annually (http://wdr.water.usgs.gov/). Multi-parameter probes adapted for low-ionic strength waters measured water temperature, $\mathrm{DO}, \mathrm{pH}$, and specific conductance. The section, "QualityAssurance Data," describes calibration for these instruments. Field measurements periodically were collected along the cross section of the stream at monitor locations to ensure that the spot measurements were representative of stream conditions.

The dates and sites of monitor deployments are listed in table 4 . To capture the daily minimum and maximum values, field crews collected instantaneous measurements of water temperature, $\mathrm{DO}$, and $\mathrm{pH}$ between $6: 30$ and 8:30 a.m. and between 3:00 and 7:00 p.m. These periods were based on data from existing continuous water-quality monitors in the basin and data from Anderson and Carpenter (1998). Crews also deployed internally logging waterquality sondes overnight at some sites to better document the timing and magnitude of the temperature, $\mathrm{DO}$, and $\mathrm{pH}$ minimums and maximums. To ensure continuous fieldparameter data at the monthly sampling sites, three new continuous water-quality monitors were established during the drawdown and restoration period: Lake Creek about $6 \mathrm{mi}$ downstream of Diamond Lake at Highway 138 (LAKE138), the inflow to Toketee Lake (TOKLKI), and the North Umpqua River above Copeland Creek (COPEG) (tables 4 and 5). Continuous monitor data are available online at the USGS Oregon Water Science Center Data Grapher, http://or.water.usgs.gov/grapher/.

\section{Streamflow}

Instantaneous and continuously recorded discharge was measured according to standard USGS guidelines (Rantz and others, 1982;
Kennedy, 1983; Buchanan and Somers, 1984). Douglas County provided discharge data from two streamgages (NUBWC and ROCKM) (Brian Connors, Douglas County, written commun., 2006).

\section{Algal Samples and Laboratory Analysis}

At 11 locations in 2005 and 2007, field crews collected one periphyton sample from cobble substrates for biomass (Chl- $a$ and ashfree dry mass [AFDM]), and species composition. In 2006, LAKEM, TOKLKI, and NURCR were also sampled (table 5). For partially wadeable sites (INLET, TOKLKI, LEM1G, BSODA, COPEG, NUSTM, and NURCR), the field crews collected rocks from riffle areas in nearshore margin habitat. To help randomize rock selection, flat metal washers with short lengths of orange flagging were tossed from shore to 10 locations within the sampling zone, and the rock or cobble immediately upstream of the washer was carefully retrieved. Algal material was collected by the toprock/cylinder scrape method (Moulton and others, 2002); the resulting algae and rinse water slurry was transferred into 1-liter (L) Nalgene ${ }^{\circledR}$ bottles and placed on ice. Periphyton samples were processed in a temporary field laboratory within 10 hours of collection. Samples were briefly homogenized in a low-speed electric blender until the resulting slurry could be pipetted using a large-orifice pipettor with a trimmed tip. The slurry was poured into a churn splitter for subsampling. Algal biomass samples ranging from 5 to 10 milliliters $(\mathrm{mL})$ were pipetted while churning, then transferred onto 47- millimeter ( $\mathrm{mm})$ glass-fiber filters $(\mathrm{GF} / \mathrm{F})$ under vacuum pressure using a plastic filtration apparatus. Chl- $a$ samples were analyzed fluorometrically with acid correction and AFDM gravimetrically using standard methods (American Public Health Association, 1989) at the ODEQ laboratory in Portland, Oregon.

Subsamples for algal species identification and enumeration were preserved by mixing 95$\mathrm{mL}$ of the algal slurry with 5-mL of full-strength 
buffered formalin (5 percent final preservative concentration). The Philadelphia Academy of Natural Sciences (ANSP) counted live cells, verified by the presence of chloroplasts or viable protoplasm within the cells, to determine cell density and biovolume of diatoms and soft algae (green, blue-green, red, and golden algae) for samples collected in 2005-07 using protocols outlined in Charles and others (2002). In 199495 samples, Aquatic Analysts (Friday Harbor, WA) analyzed algal samples for taxonomic composition and enumeration (cell density and biovolume) using protocols outlined in Anderson and Carpenter (1998). For quality assurance, ANSP analyzed preserved slides from three sites sampled in 1995: NURCR, COPEG, and STEAM.

Aquatic Analysts and ANSP provided different levels and methods of algal taxonomic analysis, which affected comparisons between the 1995 and 2005-07 samples, including these quality-assurance samples. Aquatic Analysts identified 100 individual cells or colonies per sample, whereas ANSP enumerated 300 cells per sample in the initial scan, followed by a more detailed taxonomic evaluation of acid-cleaned diatoms. ANSP counted between 365 and 600 diatom frustules per sample. Because of these differences, care should be taken when comparing samples collected in 1995 with those from 2005-07. Comparisons among the 2005, 2006, and 2007 algae samples are straightforward because all were analyzed by the ANSP.

\section{Data Analysis}

\section{Water Chemistry}

Two water-chemistry datasets from the 1995, 2005, and 2007 time periods were compiled: the first includes data from all sites, and a second contains data from only the "monthly" sampling sites-LAKEM/LAKE138, TOKLKI, COPEG, and NURCR (appendix tables $\mathrm{C} 1$ and $\mathrm{C} 2$ ).
To improve comparability between years, nutrient results less than reporting levels (nondetections) were assigned values of one-half the lowest reporting level for all years. The censored values, in milligrams per liter, were: nitrate $\left(\mathrm{NO}_{3}\right), 0.0025$; ammonium $\left(\mathrm{NH}_{4}\right), 0.005$; soluble reactive phosphorus (SRP), 0.0025; total Kjeldahl nitrogen (TKN), 0.03; total phosphorus (TP), 0.005; and total and dissolved organic carbon (TOC and DOC), 0.5. Implications of these substitutions occur in nutrient load calculations and in statistical summaries, and are discussed in the text, as applicable.

Water-chemistry data were compared to ODEQ water-quality standards for water temperature, $\mathrm{DO}$, and $\mathrm{pH}$ (table 6), or to applicable nutrient criteria suggested by the U.S. Environmental Protection Agency (EPA) for streams and rivers in the Cascade Mountain Ecoregion (table 7). ODEQ modified the water temperature and DO standards in 2010, linking them to the presence of salmon and steelhead and spawning in a given reach. For this report, data are compared to the most recent ODEQ water-quality standards, including the revisions from 2010. 
Table 6. State of Oregon water quality standards (numeric criteria) for temperature, dissolved oxygen, and $\mathrm{pH}$ for indicated reaches in the North Umpqua River basin, Oregon.

[Data Sources: Water temperature standard: http://www.deq.state.or.us/wq/pubs/imds/Temperature.pdf, accessed December 9, 2010; Dissolved oxygen standard: http://www.deq.state.or.us/wq/rules/div041/table21.pdf, accessed December 9, 2010; pH standard: http://arcweb.sos.state.or.us/pages/rules/oars_300/oar_340/340_041.html, accessed December 9, 2010. Abbreviations: mg, milligrams]

River reach (fish uses designation)

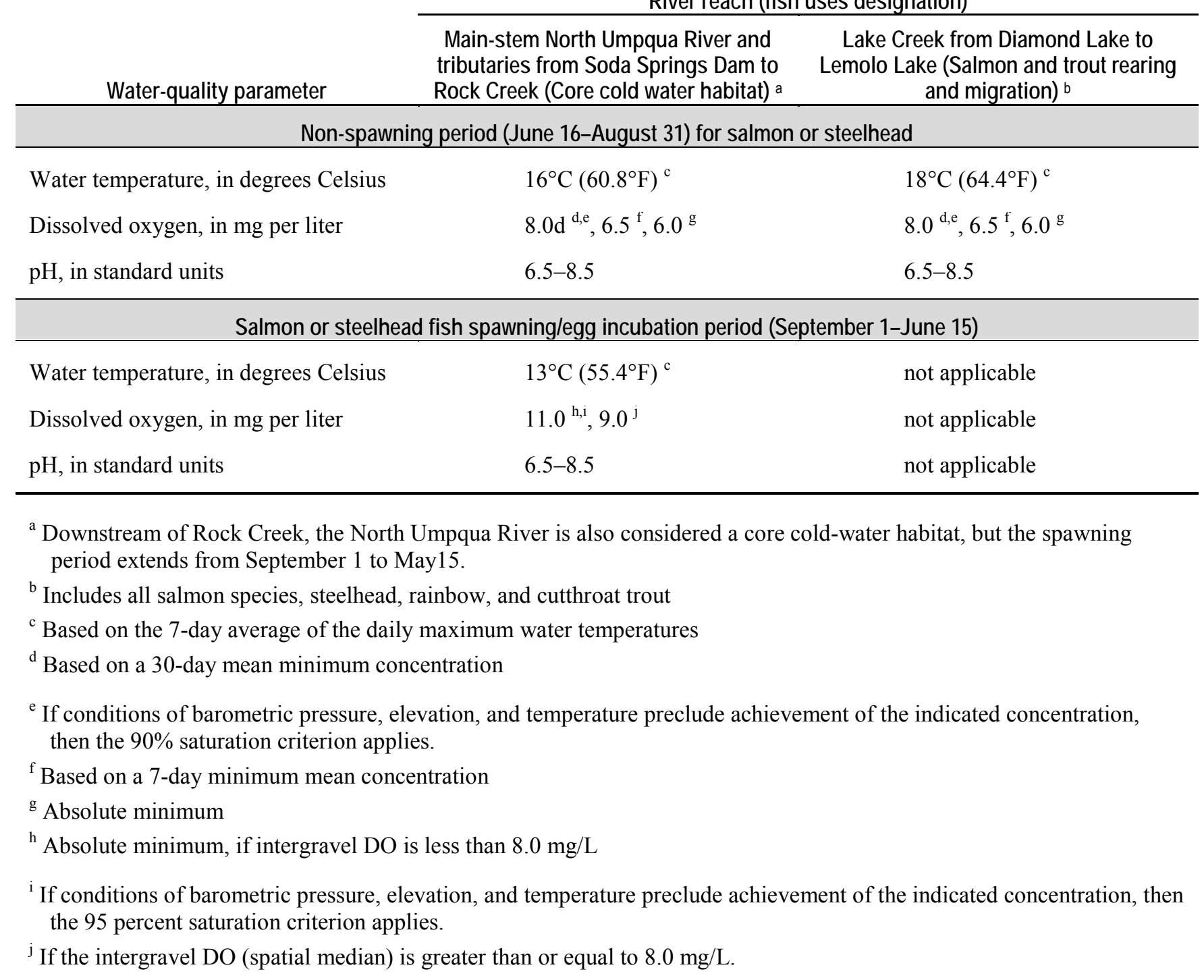


Table 7. Concentrations suggested for reference streams and as criteria for nutrients and algal biomass to prevent nuisance conditions in the North Umpqua River basin, Oregon.

[Nutrient concentrations are reported in milligrams per liter and periphyton chlorophyll- $a$ values are reported in milligrams per square meter. Abbreviations: < MRL, less than the method reporting level; --, not explicitly defined but included in the 0.1 $\mathrm{mg} / \mathrm{L}$ for dissolved inorganic nitrogen; EPA, United States Environmental Protection Agency.]

\begin{tabular}{|c|c|c|c|c|c|c|c|}
\hline \multicolumn{8}{|c|}{ Suggested nutrient reference conditions for Cascade Range streams (EPA) } \\
\hline Nutrient & $\begin{array}{l}\text { Number of } \\
\text { sites }\end{array}$ & Minimum & Maximum & \multicolumn{4}{|c|}{$\begin{array}{l}\text { Reference condition } \\
\text { (seasonal average) }\end{array}$} \\
\hline Nitrite-plus-nitrate $\left(\mathrm{NO}_{2}+\mathrm{NO}_{3}\right)$ & 75 & $<$ MRL & $1.91 \mathrm{mg} / \mathrm{L}$ & \multicolumn{4}{|c|}{$0.005 \mathrm{mg} / \mathrm{L}$} \\
\hline Total Kjeldahl nitrogen (TKN) & 65 & $<\mathrm{MRL}$ & $0.95 \mathrm{mg} / \mathrm{L}$ & \multicolumn{4}{|c|}{$0.050 \mathrm{mg} / \mathrm{L}$} \\
\hline Total nitrogen $(\mathrm{TN})$ & 27 & $<\mathrm{MRL}$ & $2.86 \mathrm{mg} / \mathrm{L}$ & \multicolumn{4}{|c|}{$0.055 \mathrm{mg} / \mathrm{L}$} \\
\hline Total phosphorus (TP) & 95 & $<\mathrm{MRL}$ & $0.24 \mathrm{mg} / \mathrm{L}$ & \multicolumn{4}{|c|}{$0.009 \mathrm{mg} / \mathrm{L}$} \\
\hline \multicolumn{8}{|c|}{ Nutrient and algal criteria } \\
\hline & & \multicolumn{6}{|c|}{ Maximum value (this study) } \\
\hline & & \multicolumn{2}{|c|}{1995} & \multicolumn{2}{|c|}{2005} & \multicolumn{2}{|c|}{2007} \\
\hline Nutrient enrichment indicators & $\begin{array}{l}\text { Suggested } \\
\text { criteria }^{a}\end{array}$ & $\begin{array}{l}\text { Wild and } \\
\text { Scenic } \\
\text { River } \\
\text { Reach }{ }^{b}\end{array}$ & Tributary & $\begin{array}{l}\text { Wild and } \\
\text { Scenic } \\
\text { River } \\
\text { Reach }{ }^{b}\end{array}$ & Tributary & $\begin{array}{l}\text { Wild and } \\
\text { Scenic } \\
\text { River } \\
\text { Reach }{ }^{b}\end{array}$ & Tributary \\
\hline Nitrite-plus-nitrate $\left(\mathrm{NO}_{2}+\mathrm{NO}_{3}\right)$ & -- & $<0.005$ & 0.039 & 0.0055 & 0.040 & 0.010 & 0.114 \\
\hline $\begin{array}{l}\text { Dissolved inorganic nitrogen } \\
\left(\mathrm{NO}_{2}+\mathrm{NO}_{3}+\mathrm{NH}_{4}\right)\end{array}$ & 0.1 & $<0.005$ & 0.039 & 0.026 & 0.059 & 0.026 & 0.114 \\
\hline Soluble reactive phosphorus (SRP) & $0.01-0.015$ & 0.033 & 0.036 & 0.050 & 0.074 & 0.042 & 0.019 \\
\hline Total phosphorus (TP) & $0.01-0.09$ & 0.037 & 0.039 & 0.053 & 0.080 & 0.060 & 0.030 \\
\hline Periphyton chlorophyll- $a$ (Chl- $a$ ) & $100-150^{\mathrm{c}}$ & 58 & 82 & 91 & 133 & 121 & 128 \\
\hline \multicolumn{8}{|c|}{$\begin{array}{l}\text { a Nutrient and algal criteria for streams in the Western Forested Mountains (Nutrient Ecoregion II), Cascade Range } \\
\text { (Subecoregion 4), Nutrient Criteria Technical Guidance Manual, U.S. Environmental Protection Agency, EPA-822-B-00- } \\
\text { 002, July 2000. }\end{array}$} \\
\hline
\end{tabular}


Algae

No regulatory guidelines exist for periphyton algal biomass. For purposes of comparison, we used concentrations for reference streams and criteria for nutrients and algal biomass suggested to prevent nuisance conditions in rivers like the North Umpqua River (table 7). We evaluated possible changes in water quality and algal conditions between 1995 and 2005 by analyzing patterns in periphyton biomass, species composition, algal biovolume, cell density, and indicator species or guilds (Porter, 2008). Using data from 2005 and 2007, we evaluated changes associated with the Diamond Lake restoration project by analyzing these same indicators longitudinally among sites and at individual sites over time.

The different expressions of algal species abundance, whether as absolute cell density (in cells per square centimeter), biovolume (in cubic micrometers per square centimeter), percent density, or percent biovolume, did not always produce consistent results for the algal indicator guilds, due mostly to a large range in cell sizes and growth morphologies of the dominant taxa within each sample. Density results were skewed by high numbers of fast growing small-celled taxa (small diatoms and blue-green algae) that often dominated the metric values based on density. In contrast, biovolume results were skewed by larger-sized taxa, including filamentous green algae, several species of highbiomass-forming stalked diatoms, and other taxa that often dominated metric values based on biovolume. Analyses focused on biovolume because, by accounting for size, biovolume more closely represents biomass, which is important in supporting river food webs, and is a concern because high biomass can produce nuisance aesthetic and habitat conditions, and can impact dissolved oxygen and $\mathrm{pH}$. We selected percent biovolume as the metric of choice because the percent (relative) expression is most characteristic of the assemblage quality. Metric values based on the total (absolute) biovolume, on the other hand, are strongly dictated by the biomass levels at the time of sampling, which can be variable throughout the season.

Harmonization-Prior to the multivariate analyses, algal species data from Aquatic Analysts and ANSP from 1995 and 2005-07 were merged or "harmonized" into one dataset after adjusting for taxonomic inconsistencies between the two contractors. Most of the inconsistencies between contactors were identified during a quality-assurance comparison of three samples of acid-cleaned diatoms in 1995 (see section, "Algal Sampling and Laboratory Analysis "). For the harmonized dataset, certain algal taxa were combined into more generalized, less specific classification groups. As necessary, multiple subspecies were combined or "lumped" into species; multiple species were grouped into one genus, and so forth. For example, in the harmonized dataset, the nitrogen-fixing diatom genus Epithemia spp. includes Epithemia adnata, Epithemia geoppertiana, Epithemia sorex, Epithemia turgida, and Epithemia turgida var. westermannii (see footnotes in appendix tables D2 and D3). As another example, in the harmonized dataset, the Rivulariaceae family represents multiple genera of heterocystousbearing, nitrogen-fixing filamentous cyanobacteria, including Calothrix, Tolypothrix, and Rivularia. In this way, uncertainties between contractor taxonomy were reduced so that meaningful comparisons could be made between 1995 and 2005.

Multivariate Analyses-Periphyton species composition (both diatoms-only and soft algae datasets) were analyzed using multivariate statistical software (PRIMER-E, version 6: Clarke and Gorley, 2006). Our analyses focused on these questions:

- Did algal biomass or species composition change between 1995 and 2005?

- Did biomass or species composition change in response to the Diamond Lake restoration project between 2005 and 2006-07?

- Which factors best explain patterns in algal species composition each year? 
Non-metric multidimensional scaling (NMDS) ordination was used to examine patterns in algal species composition. The NMDS algorithm iteratively distributes samples in the plots according to their similarity; samples having greater similarity plot closer to each other, and samples that are less similar plot farther apart (Clarke and Gorley, 2006). The plots are based on Bray-Curtis similarity, which was determined after square-root transforming the percent abundance data for each sample. This statistical method reduces the complex multidimensional nature of ecological data to a reduced set of axes to facilitate interpretations.

We analyzed associations between the algal species composition and the environmental data matrix with the Bio-Env Stepwise (BEST) procedure, which tests individual and groups of variables for significance in explaining variations in algal species composition. These analyses also focused on either diatoms or soft algae.

Autecological Guilds-Indicator species, or guilds, are groups of taxa whose special attributes reliably reflect environmental conditions. Because algae are sensitive to nutrient concentrations, $\mathrm{pH}, \mathrm{DO}$, conductivity, and other parameters, they provide a timeintegrated record of stream conditions that is complementary to instantaneous water-quality measurements (Carrick and others, 1988; Lowe and Pan, 1996; Porter, 2008). The signal from algal guilds may provide a more accurate, longer-term assessment of water-quality or habitat conditions than discrete sampling. For example, nutrients can produce high algal biomass, but uptake by periphytic algae can reduce ambient nutrient concentrations to less than laboratory detection limits. In such a case, the abundance, or biomass, of eutrophic algae would be a more accurate indication of nutrient enrichment over time than instantaneous watercolumn nutrient concentrations.
Guild assignments are based on autecological classifications given in Porter (2008). Some guilds are based on diatoms only, whereas others (N-fixers, alkaliphilic, and eutrophic taxa) include only soft algae. In the North Umpqua datasets, guilds with the highest abundances were:

- Nitrogen-fixing algae (NF), which have the ability to convert atmospheric nitrogen $\left(\mathrm{N}_{2}\right)$ into biologically available $\mathrm{NH}_{4}$;

- Nitrogen-heterotrophic (NH) algae, which have the ability to assimilate organic nitrogen as a source of both nitrogen and energy;

- Eutrophic taxa (E), which prefer or require high nutrient concentrations;

- Alkaliphilic taxa (Alk-P), which prefer alkaline waters, greater than $7 \mathrm{pH}$ units; and

- Alkalibiontic taxa (Alk-B), which prefer even more alkaline waters (greater than $7 \mathrm{pH}$ units for diatoms and greater than $8 \mathrm{pH}$ units for soft algae) (Porter, 2008; van Dam and others, 1994; Lowe, 1974).

The relative proportion (percentage) of each guild at a site is a better indicator of waterquality conditions than the overall guild abundance. Because the total cell density or biovolume of algae at a site affects the total abundance of a particular guild, the abundance may be solely due to greater biomass. Percentage analysis adjusts for those seasonal or annual variations in algal biomass.

Guild percentages were based on classified taxa only, and did not include taxa with no published autecologies for that trait. For example, the percentage of eutrophic taxa was based on all taxa for which a trophic status was assigned, but did not include taxa for which a trophic status was not available. Further complicating matters, guilds are not mutually exclusive and one particular taxon could be assigned into multiple guilds (for example, many eutrophic taxa also are nitrogen heterotrophs). 


\section{Quality-Assurance Data}

This study included several qualityassurance measures for sample collection, processing, and analysis, including blanks, replicates, splits, and standard reference samples.

\section{Nutrients}

Seven equipment blank samples were submitted to check for contamination during sample collection and laboratory analysis. Samples were prepared in the field by passing certified nutrient-free water through all sampling equipment into clean sample bottles. Three of the equipment blanks had detectable concentrations of nitrogen or carbon. In July 2005, the ODEQ laboratory detected $\mathrm{NH}_{4}$ at $0.015 \mathrm{mg} / \mathrm{L}$ in one sample, and $\mathrm{NO}_{3}$ at $0.0024 \mathrm{mg} / \mathrm{L}$ in another. The ODEQ Laboratory minimum reporting level (MRL) for dissolved $\mathrm{NH}_{4}$ was $0.02 \mathrm{mg} / \mathrm{L}$; detection of $\mathrm{NH}_{4}$ in the blank confirms that reported concentrations of less than $0.02 \mathrm{mg} / \mathrm{L}$ are suspect. The low $\mathrm{NO}_{3}$ detection in the blank was much less than the MRL of $0.005 \mathrm{mg} / \mathrm{L}$ and most of the detected concentrations. Based on these blank detections, $\mathrm{NH}_{4}$ and $\mathrm{NO}_{3}$ detections close to the ODEQ MRLs were interpreted cautiously. In May 2006, TOC was detected in a blank sample at the MRL concentration of 1 $\mathrm{mg} / \mathrm{L}$. One "transport" blank sample resulted in no detections. Replicate nutrient sample concentrations generally were within 20 percent of each other, although differences exceeded this amount for some low concentrations of $\mathrm{NH}_{4}$, $\mathrm{NO}_{3}$, TKN, TP, DOC, and TOC (bolded pairs in appendix table B2). The one standard reference sample submitted in 2005 for nutrients showed good agreement between expected and reported values (appendix table B3).

\section{Algae}

Quality-assurance samples for algae included independently collected replicate samples and split samples for periphyton biomass (Chl- $a$ and AFDM), and split samples for species identification. As described in the section "Algal Samples and Laboratory Analysis," three samples were analyzed in 1995 by Aquatic Analysts and ANSP to identify potential differences in taxon names assigned by the two contractors.

The often-patchy growth of periphyton (Cazaubon and others, 1995) increases variability in algal sampling. To assess sampling variation, we separately collected and composited replicate quality-assurance samples for algal biomass (Chl- $a$ and AFDM) and species composition from one location (NURCR). The samples are named NURCR1 and NURCR2 in this report. Additionally, Chl- $a$ and AFDM samples from all sites were analyzed in triplicate to characterize laboratory variability.

The independently collected replicate samples for algal Chl- $a$ and AFDM showed much better agreement in 2005-07 than in 1995, with sample values within a few percentage points of each other (appendix table B4). The median percent relative standard deviation for triplicate biomass samples had acceptable reproducibility, 5.3 and 4.8 percent for Chl- $a$ and AFDM, respectively (appendix table B5). Samples collected from TOKLKI (2007) and NUBWC (2005) had somewhat higher variability that was likely caused by subsampling irregularities from the presence of either filamentous green algae (Cladophora glomerata) or diatoms with large amounts of stalked material (large Gomphoneis and Cymbella taxa).

Anderson and Carpenter (1998) presented the quality-assurance results for species identification and enumeration samples from 1995. Reproducibility between replicate split samples for 2005-07 was acceptable, ranging from 69 to 82 percent similarity for all-taxa cell density (appendix table B7). Replicate samples in 2007 had lower percent similarity (55 percent) for the all-taxa biovolumes comparison because Cladophora glomerata appeared in the first replicate sample only, and the blue-green algal Pleurocapsa minor and filamentous green Ulothrix sp. appeared in the second sample only. 
The percent similarity in 2007 for diatoms in these replicates was much higher at 72 percent. The similarities for percent biovolume of soft algae in replicate samples from 2005 and 2007 were 17 percent and 37 percent, respectively. The often-patchy growth of Homoeothrix varians, Spirogyra sp., and Gongrosira sp., which appeared in just one of these samples, was the probable source of the large difference.

\section{Hydrologic, Water-Quality, and Benthic-Algae Conditions, 1995-2007}

The following section presents data from synoptic surveys in 2005-07, and evaluates conditions immediately prior to, during, and following the Diamond Lake restoration project to identify the short-term effects of the restoration. To evaluate longer-term patterns, the synoptic data were compared with data from 1995 and continuous monitor data from 200007. Data from two distinct, independent periods prior to the restoration project allowed for evaluation of the inherent variability in conditions between synoptic surveys, and possible long-term changes unrelated to the restoration project at Diamond Lake.

\section{Hydrologic Conditions}

Anderson and Carpenter (1998) noted the importance and interaction of four hydrologic factors in the Wild and Scenic Reach: (1) seasonal snowfall and snowmelt cycles at upper elevations, (2) upper basin groundwater input, (3) cycles of winter rain and dry summers in lower elevations, and (4) flow modifications and diversions from the hydroelectric project. These four factors also controlled river conditions during the 2005-07 period of the Diamond Lake restoration, though they varied in relative importance from year to year. In both periods, these hydrologic factors resulted in a relatively high, stable flow in the North Umpqua River main stem during summer, despite low flows in most tributaries.
Anderson and Carpenter (1998) computed a water budget based on data from the 1995 survey to identify possible unmeasured sources of water or nutrients in the Wild and Scenic Reach. That study accounted for major sources of water, except for moderate accretion from groundwater and (or) unmeasured tributaries in the reach between Steamboat Creek and Glide. Although field crews measured discharge at almost all sampling sites during the 2005 and 2007 synoptic surveys, variable hydroelectric project releases made it impossible to balance the water budget completely. The analysis that follows discusses the implications of this uncertainty, but otherwise assumes that unmeasured water or nutrient sources were similar in 2005-07 and 1995, and were insignificant.

\section{Patterns in Hydrology in 1995-2007 and Changes} Associated with the New Hydroelectric License

Discharge in the Wild and Scenic Reach is dominated by hydroelectric project flow releases, and is best represented by the streamgage upstream of Copeland Creek (COPEG, map no. 11, fig. 1), the only USGS-operated main-stem streamgage between Winchester Dam near the Interstate Hwy 5 crossing and Soda Springs. The hydrograph at COPEG (fig. 3) illustrates the inter-annual variability of upper-basin snowpack and highlights the effects of specific events such as the November 1996 warm-rain-on-snow event and the low snow packs of 1994 and 2001. 


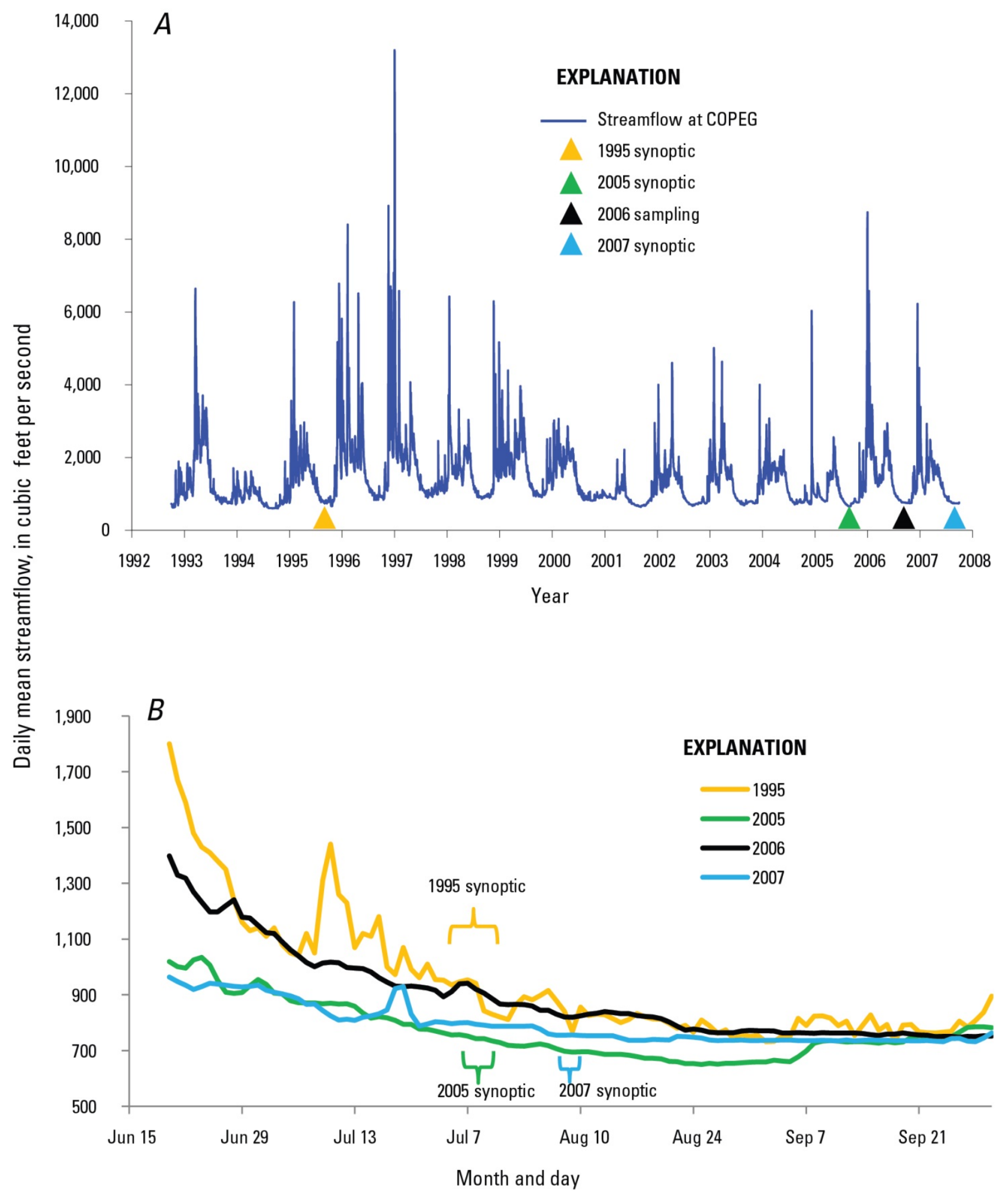

Figure 3. Graphs showing streamflow in the North Umpqua River above Copeland Creek (COPEG), Oregon, for (A) water year 1993-2007 and (B) summers of 1995 and 2005-07. 
Examination of the discharge record shows reduced watershed production from 2001 to 2007 compared to the previous decade. Flow duration statistics at COPEG (table 8) support this conclusion; during 2000-2010, median flows were reduced by $176 \mathrm{ft}^{3} / \mathrm{s}$ in July and $136 \mathrm{ft}^{3} / \mathrm{s}$ in
August, or about 15-17 percent compared with the previous decade. The nearby Klamath River Basin mirrored this pattern, which Mayer and Naman (2011) attributed to reduced snowpack and earlier snowmelt runoff.

Table 8. Comparisons of flow-duration statistics for the North Umpqua River above Copeland Creek for 1990-2000 and 2000-10.

[Percentile exceedance is the percent of time that the indicated discharge value is exceeded. For example, a 95 percent exceedance value represents a low flow that is exceeded 95 percent of the time for the indicated month. Discharges are in cubic feet per second.]

\begin{tabular}{|c|c|c|c|c|c|c|c|c|c|c|}
\hline \multirow[b]{3}{*}{ Period (water years) } & \multicolumn{10}{|c|}{ Discharge at indicated exceedance value (percentile) } \\
\hline & \multicolumn{2}{|c|}{$95^{\text {th }}$} & \multicolumn{2}{|c|}{$75^{\text {th }}$} & \multicolumn{2}{|c|}{$50^{\text {th }}$} & \multicolumn{2}{|c|}{$25^{\text {th }}$} & \multicolumn{2}{|c|}{$5^{\text {th }}$} \\
\hline & July & August & July & August & July & August & July & August & July & August \\
\hline $1990-2000$ & 645 & 590 & 870 & 762 & 1,060 & 893 & 1,140 & 964 & 1,570 & 1,150 \\
\hline $2000-2010$ & 726 & 660 & 804 & 711 & 884 & 757 & 993 & 836 & 1,230 & 995 \\
\hline $\begin{array}{l}\text { Percent Change } \\
\text { (1990s to 2000s) }\end{array}$ & $13 \%$ & $12 \%$ & $-8 \%$ & $-7 \%$ & $-17 \%$ & $-15 \%$ & $-13 \%$ & $-13 \%$ & $-22 \%$ & $-13 \%$ \\
\hline
\end{tabular}

Mean annual streamflow at COPEG during the 2006 water year was $98 \mathrm{ft}^{3} / \mathrm{s}$ higher than during 1950-2009 (1,555 $\mathrm{ft}^{3} / \mathrm{s}$ compared to 1,457 $\left.\mathrm{ft}^{3} / \mathrm{s}\right)$ (http://wdr.water.usgs.gov/, accessed November 3, 2010). This surplus is attributable to a large snowpack in 2006 and the additional water released from Diamond Lake in advance of the 2006 rotenone application.

Streamflow for each site measured during each of the three synoptic sampling events is shown in table 9 . Flows were about $200 \mathrm{ft}^{3} / \mathrm{s}$, or 25 percent higher in the Wild and Scenic main stem during the 1995 synoptic compared with 2005 (fig. 3). Tributary flows were on average 37 percent lower in 2005 compared with 1995 (table 10).

In 2007, flows in the Wild and Scenic Reach were 13 percent higher compared with 2005 (table 10) owing to a moderate storm 2 weeks prior to sampling, a large snowpack, and cooler air temperatures. The daily maximum air temperatures at the Toketee airstrip, $3 \mathrm{mi}$ south of Toketee Lake, were $7^{\circ} \mathrm{C}$ lower during the 2007 synoptic than in 2005 (Natural Resources Conservation Service, 2013). The snowpack differences did not increase summer streamflow from tributaries entering the Wild and Scenic Reach, however, because these streams originate in the lower-elevation Western Cascades, where snow accumulation is only minor. During the 2005 and 2007 synoptic surveys, streamflow in these tributaries (not including Lake Creek) averaged 28 and 60 percent less, respectively, than in 1995 (data for 2005 versus 1995 are not shown). The potential implications of the lower flows and their influence on nutrient concentrations are discussed further below. 
Table 9. Site-specific streamflow conditions during the summer synoptic sampling events in the North Umpqua River basin, Oregon, 1995, 2005, and 2007.

[Streamflow values are in cubic feet per second. Abbreviations: --, not measured]

\begin{tabular}{|c|c|c|c|c|}
\hline \multirow[b]{2}{*}{ Site Code } & \multirow[b]{2}{*}{ Site Name } & \multicolumn{3}{|c|}{ Year } \\
\hline & & 1995 & 2005 & 2007 \\
\hline \multicolumn{5}{|c|}{ Bypass reaches in the hydroelectric project area } \\
\hline LEM1T/G & North Umpqua River below Lemolo Lake & $38^{\mathrm{a}}$ & 38 & 88 \\
\hline TOKET & North Umpqua River below Toketee Lake & 18 & 47 & 91 \\
\hline SODAT & North Umpqua River below Soda Springs & 35 & -- & -- \\
\hline TOKLKI & North Umpqua River above Toketee Lake & 238 & 277 & 294 \\
\hline \multicolumn{5}{|c|}{ Main-stem North Umpqua River } \\
\hline INLET & North Umpqua River above Lemolo Lake near Inlet Campground & 289 & 257 & 300 \\
\hline BSODA & North Umpqua River below Soda Springs Powerhouse & 881 & 630 & 784 \\
\hline COPEG & North Umpqua River above Copeland Creek & 943 & 747 & 769 \\
\hline NUAPC & North Umpqua River at Apple Creek & 927 & 644 & 862 \\
\hline MOTTB & North Umpqua River at Mott Bridge & 979 & 782 & 780 \\
\hline NUBWC & North Umpqua River at Wright Creek below Steamboat Creek & 1,019 & 857 & 960 \\
\hline NURCR & North Umpqua River above Rock Creek & 1,140 & 733 & 862 \\
\hline NUGLD & North Umpqua River near Glide & 1,170 & 838 & 836 \\
\hline \multicolumn{5}{|c|}{ Tributaries } \\
\hline LAKEM & Lake Creek above Lemolo Lake & 57 & 12 & 27 \\
\hline BOULM & Boulder Creek near mouth & 7.7 & 9.5 & 5.2 \\
\hline COPEM & Copeland Creek near mouth & 23 & 13 & 8.2 \\
\hline CALFM & Calf Creek near mouth & 7.8 & 4.6 & 2.5 \\
\hline PANTM & Panther Creek near mouth & 6.2 & 3.4 & 1.7 \\
\hline STEAM & Steamboat Creek at gage & 103 & 66 & 40 \\
\hline ROCKM & Rock Creek near mouth & 80 & 45 & -- \\
\hline LITGL & Little River near Glide & 60 & 40 & 27 \\
\hline
\end{tabular}

\footnotetext{
${ }^{a}$ Streamflow was measured upstream at LEM1T.
} 
Table 10. Average percent change in streamflow, select field parameters, and nutrients at sites located in the Wild and Scenic Reach, tributaries, and the Diamond Lake Restoration Affected Reach, for 1995-2005 and 2005-2007.

[Average percent change for sites within the group. A range is given for the number of sites in the Wild and Scenic Reach and Tributaries groups because some sites were not sampled every year. Abbreviations: n, number of sites in each group; $\%$, percent]

\begin{tabular}{|c|c|c|c|c|c|c|}
\hline \multirow[b]{3}{*}{ Parameters } & \multicolumn{6}{|c|}{ Average percent change $^{a}$} \\
\hline & \multicolumn{3}{|c|}{$1995-2005$} & \multicolumn{3}{|c|}{ 2005-2007 } \\
\hline & $\begin{array}{l}\text { Wild and } \\
\text { Scenic } \\
\text { Reach b } \\
(n=5-6)\end{array}$ & $\begin{array}{c}\text { Tributaries } \\
(\mathrm{n}=4-8)\end{array}$ & $\begin{array}{c}\text { Diamond Lake } \\
\text { Restoration } \\
\text { Affected } \\
\text { Reach } \\
(n=11) \\
\end{array}$ & $\begin{array}{l}\text { Wild and } \\
\text { Scenic } \\
\text { Reach b } \\
(n=5-6)\end{array}$ & $\begin{array}{c}\text { Tributaries } \\
(n=3-8)\end{array}$ & $\begin{array}{c}\text { Diamond Lake } \\
\text { Restoration } \\
\text { Affected } \\
\text { Reach } \\
(\mathrm{n}=11) \\
\end{array}$ \\
\hline Streamflow & $-25 \%$ & $-37 \%$ & $-10 \%$ & $13 \%$ & $-19 \%$ & $42 \%$ \\
\hline Water temperature, diel minimum & $10 \%$ & $-3 \%$ & $3 \%$ & $-9 \%$ & $2 \%$ & $-3 \%$ \\
\hline Water temperature, diel maximum & $3 \%$ & $8 \%$ & $7 \%$ & $-6 \%$ & $-5 \%$ & $-5 \%$ \\
\hline $\begin{array}{l}\text { Dissolved oxygen concentration, } \\
\text { diel minimum }\end{array}$ & $-1 \%$ & $2 \%$ & $-4 \%$ & $3 \%$ & $-5 \%$ & $3 \%$ \\
\hline Dissolved oxygen, diel maximum & $3 \%$ & $6 \%$ & $1 \%$ & $0 \%$ & $-5 \%$ & $0 \%$ \\
\hline $\begin{array}{l}\text { Dissolved oxygen, percent } \\
\text { saturation, diel minimum }\end{array}$ & $-3 \%$ & $7 \%$ & $-1 \%$ & $2 \%$ & $-12 \%$ & $-2 \%$ \\
\hline $\begin{array}{l}\text { Dissolved oxygen, percent } \\
\text { saturation, diel maximum }\end{array}$ & $6 \%$ & $4 \%$ & $2 \%$ & $1 \%$ & $2 \%$ & $1 \%$ \\
\hline $\mathrm{pH}$, diel minimum & $0 \%$ & $1 \%$ & $2 \%$ & $0 \%$ & $-1 \%$ & $1 \%$ \\
\hline $\mathrm{pH}$, diel maximum & $0 \%$ & $2 \%$ & $1 \%$ & $1 \%$ & $0 \%$ & $2 \%$ \\
\hline Specific conductance & $11 \%$ & $7 \%$ & $10 \%$ & $-3 \%$ & $17 \%$ & $-1 \%$ \\
\hline Dissolved ammonium $\left(\mathrm{NH}_{4}\right)$ & $50 \%$ & $125 \%$ & $100 \%$ & $0 \%$ & $-11 \%$ & $-3 \%$ \\
\hline Total Kjeldahl nitrogen (TKN) & $43 \%$ & $6 \%$ & $34 \%$ & $-19 \%$ & $0 \%$ & $-17 \%$ \\
\hline $\begin{array}{l}\text { Dissolved nitrite plus nitrate } \\
\left(\mathrm{NO}_{2}+\mathrm{NO}_{3}\right)\end{array}$ & $50 \%$ & $436 \%$ & $160 \%$ & $61 \%$ & $46 \%$ & $34 \%$ \\
\hline Total phosphorus (TP) & $35 \%$ & $73 \%$ & $31 \%$ & $17 \%$ & $-26 \%$ & $10 \%$ \\
\hline $\begin{array}{l}\text { Soluble reactive phosphorus } \\
\text { (SRP) }\end{array}$ & $33 \%$ & $62 \%$ & $22 \%$ & $1 \%$ & $-13 \%$ & $2 \%$ \\
\hline
\end{tabular}

${ }^{a}$ Values of one-half the detection limit were substituted when nutrient concentrations were below detection (see Methods section).

${ }^{\mathrm{b}}$ Does not include BSODA site located downstream from Soda Springs Dam. 
In contrast to the tributaries and main stem, flow in two hydroelectric project bypass reaches (TOKET and TOKLKI) was higher during the 2005 synoptic (table 9). In the other bypass reach site measured, LEM1G downstream of Lemolo Lake, discharge was similar in 1995 and 2005 because the new FERC minimum in-stream flow requirements were not in effect for most reaches during summer 2005. Changes in discharge in these bypass reaches have no direct effect on total discharge in the Wild and Scenic Reach (represented by the streamgage at COPEG) because all upstream flows are combined downstream from the Soda Springs powerhouse located a short distance upstream from the gage.

Changes in Hydrology Associated with the Diamond Lake Restoration Project

Diamond Lake was drawn down to a minimum volume in order to minimize the quantity of rotenone required and to maximize the efficacy of the treatment. On November 23, 2005, Diamond Lake water was released into Lake Creek by way of a new canal downstream from the lake's normal outlet elevation. By
September 6, 2006, the lake surface was $8 \mathrm{ft}$ below normal summer elevation, and the surface area was 13 percent less than during normal summer conditions, exposing about 400 acres of sediment around the perimeter (U.S. Forest Service, 2004; Eilers and Truemper, 2010). On that date, the canal gates were closed to prevent release of rotenone to Lake Creek (fig. 4 and table 2). Discharge from Lake Creek to Lemolo Lake (LAKEM) was reduced but not eliminated because of groundwater and tributary inputs below Diamond Lake (Salinas and others, 1995; Anderson and Carpenter, 1998). ODFW opened the canal after the rotenone treatment was complete. The canal had a minor increase in discharge $\left(<0.5 \mathrm{ft}^{3} / \mathrm{s}\right)$ beginning on November 7 , 2006, and the discharge increased intermittently until water flowed consistently in the canal on November 30, 2006 (fig. 4). Discharge at LAKEM remained below normal until March 15, 2007, when the lake level rose sufficiently to produce flow from the original lake outlet. The canal was closed on March 20, 2007, restoring full flow into Lake Creek (table 2).

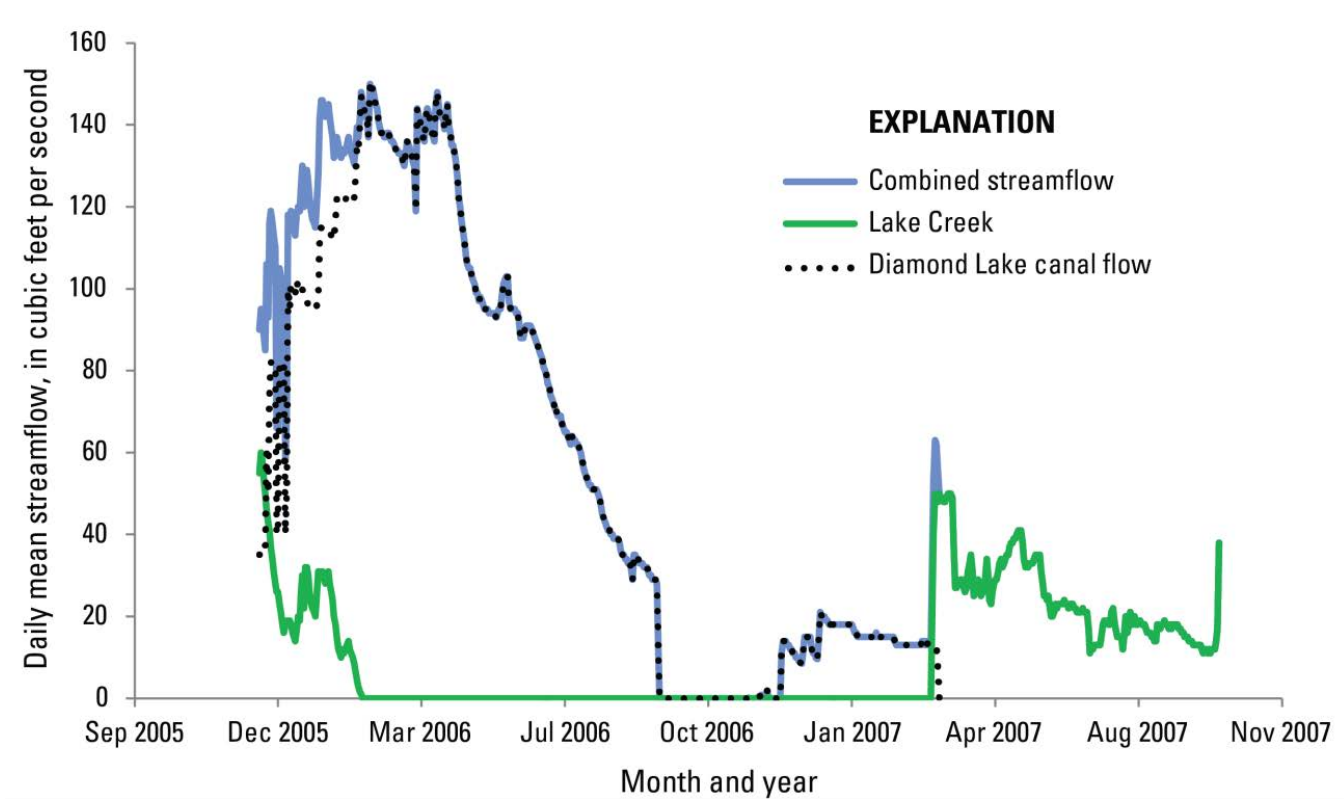

Figure 4. Streamflow in Lake Creek, Diamond Lake outflow canal, and total combined flow during and after the Diamond Lake drawdown, December 2005 to October 2007. Combined streamflow includes both Lake Creek and the canal. 
The Diamond Lake restoration project resulted in about a net 5,000 acre-ft decrease in yield to Lake Creek between water years 2006 and 2007 based on the long-term average annual discharge of almost 40,000 acre-ft (1927-2009). During water year 2006, which included the drawdown period, the total discharge of almost 62,000 acre-ft was compressed into 11 months. In 2006, the lake discharged about 22,000 additional acre-ft of water through Lake Creek and into the North Umpqua River Basin; in 2007 , as the lake was refilling, only about 13,000 acre-ft was discharged to Lake Creek (http://waterdata.usgs.gov/nwis).

\section{Water-Quality Conditions}

The synoptic surveys and the network of continuous monitors for field parameters in the North Umpqua River Basin (table 4) provide a rich dataset to evaluate these parameters, and to provide insight into algal conditions and related metabolic processes, including photosynthesis, respiration, and nutrient uptake within the main stem and tributaries.

Data collected during the synoptic surveys (fig. 5) provide a snapshot of water-quality conditions in the tributaries and main-stem North Umpqua River. Continuous monitors in the main stem North Umpqua River below Soda Springs Dam, above Copeland Creek, and near Idleyld Park, provided time series data near the top and bottom of the Wild and Scenic Reach. Neither dataset provides a full description of conditions throughout the basin, which can vary temporally and spatially. Together, they provide a more complete view of water quality in the river for the period from 1995-2007, and additional data are available from the monitors from 2000-2007 that were not analyzed for this report.

Field Parameters-Water Temperature, Dissolved Oxygen, $\mathrm{pH}$, and Turbidity

Water Temperature-Continuous data from the main-stem North Umpqua River near Idleyld Park showed that summer maximum water temperatures were similar across years, although the duration and timing of the seasonal maximums differed (fig. 6A).

The TMDL for the Umpqua River documents water temperature and cold water sources (Oregon Department of Environmental Quality, 2006). The most significant sources of summer cold water to the system are the North Umpqua River above Lemolo Lake (INLET, RM 94.8), which contributes between 250 and 300 $\mathrm{ft}^{3} / \mathrm{s}$, and the Clearwater River (RM 75.4), which contributes about $200 \mathrm{ft}^{3} / \mathrm{s}$. Both sources are dominated by influent groundwater. USGS field crews were not able to gain access to Fish Creek, which is known to be warmer than the Clearwater River because it lacks a similar cool groundwater influence (Oregon Department of Environmental Quality, 2006). However, monitoring by USFS shows Fish Creek summer water temperatures decreased by $1-3^{\circ} \mathrm{C}$ by 2006 , following implementation of increased in-stream flow requirements in the revised FERC license (table 3; U.S. Forest Service, 2009).

The continuous water-quality monitors downstream from Soda Springs (BSODA) and upstream from Copeland Creek (COPEG), near the upstream end of the Wild and Scenic Reach, showed that the daily maximum water temperatures always were less than $16^{\circ} \mathrm{C}$ in 2005-07 (U.S. Geological Survey, 2013), and thereby always met the standard in effect during the summer synoptic samplings (table 6). These temperatures indicate the abundance of relatively cold influent groundwater in the upper basin, which is combined upstream of Soda Springs Dam before entering the Wild and Scenic Reach downstream. Water temperatures steadily increased downstream of Soda Springs Dam (BSODA; fig. 5), as air temperature equilibration and flow from the tributaries contributed to daily maximum temperatures greater than $19^{\circ} \mathrm{C}$ near the downstream end of the Wild and Scenic Reach near Idleyld Park (fig. 6A). 
A. Mainstem North Umpqua River
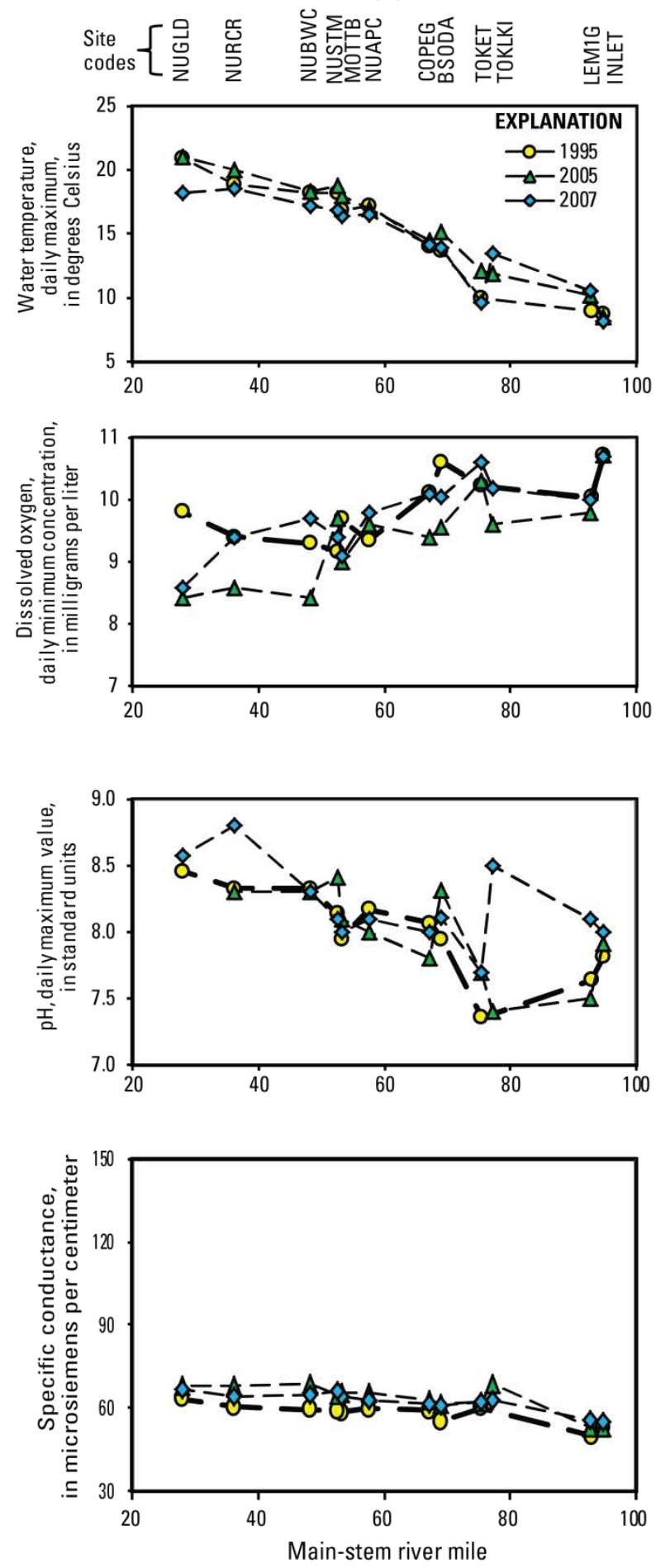

B. Tributaries
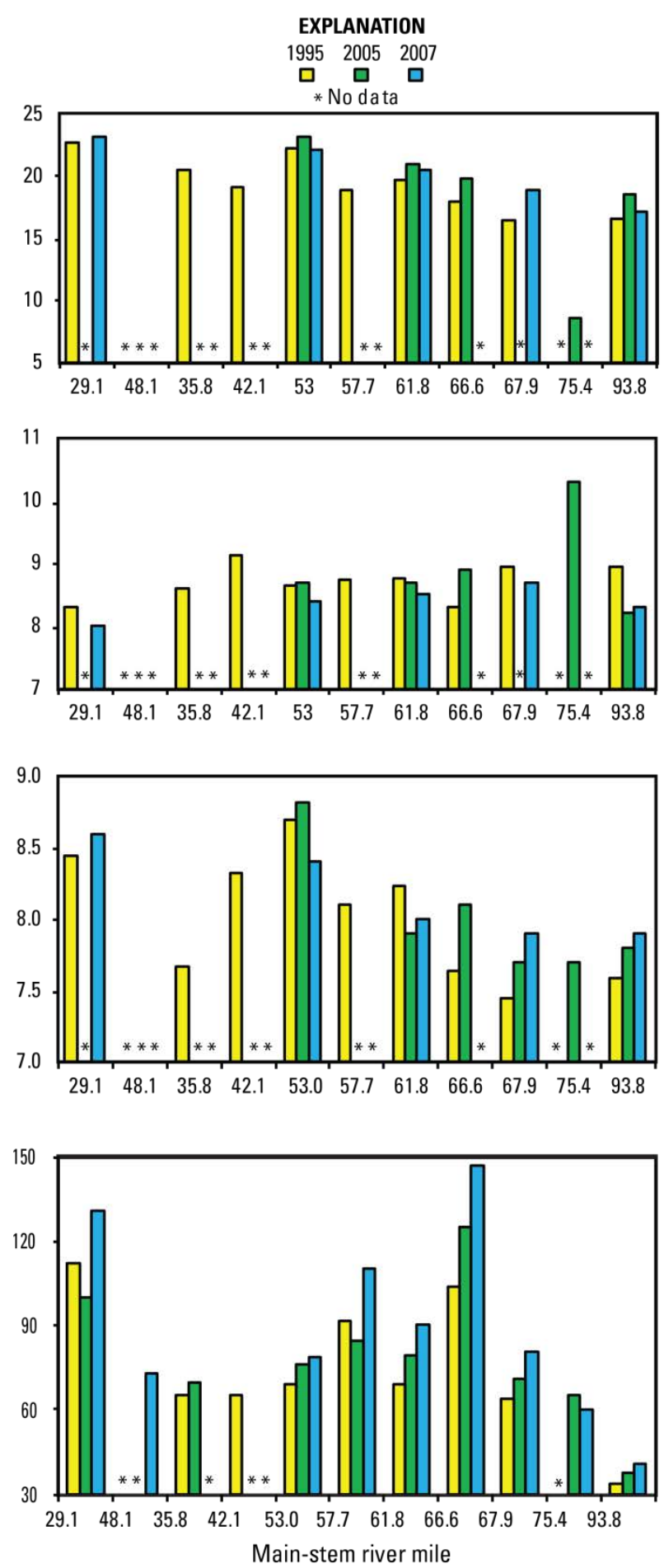

Figure 5. Selected diel field parameter values for the (A) main-stem North Umpqua River and (B) tributaries during the 1995, 2005, and 2007 synoptic surveys. See table 5 for site code definitions. 

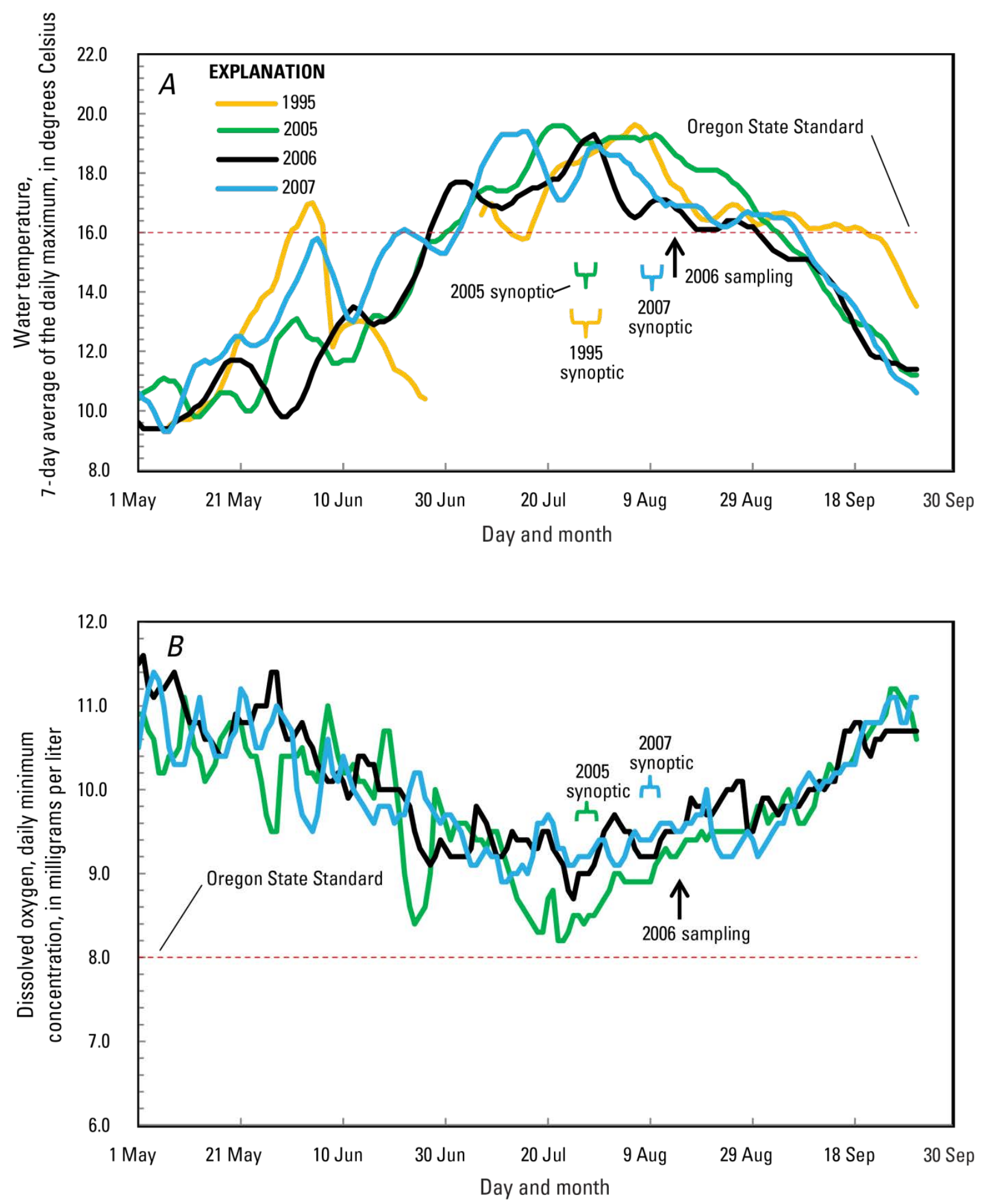

Figure 6. Continuous field parameter data from North Umpqua River near Idleyld Park (14137450), Oregon, 2005-07, showing $(A)$ the 7-day average of the daily maximum water temperatures and $(B)$ daily minimum dissolved oxygen. 1995 water temperature data from a monitor located about 0.25 mi downstream of the current location above Rock Creek. Figure continues on next page. 


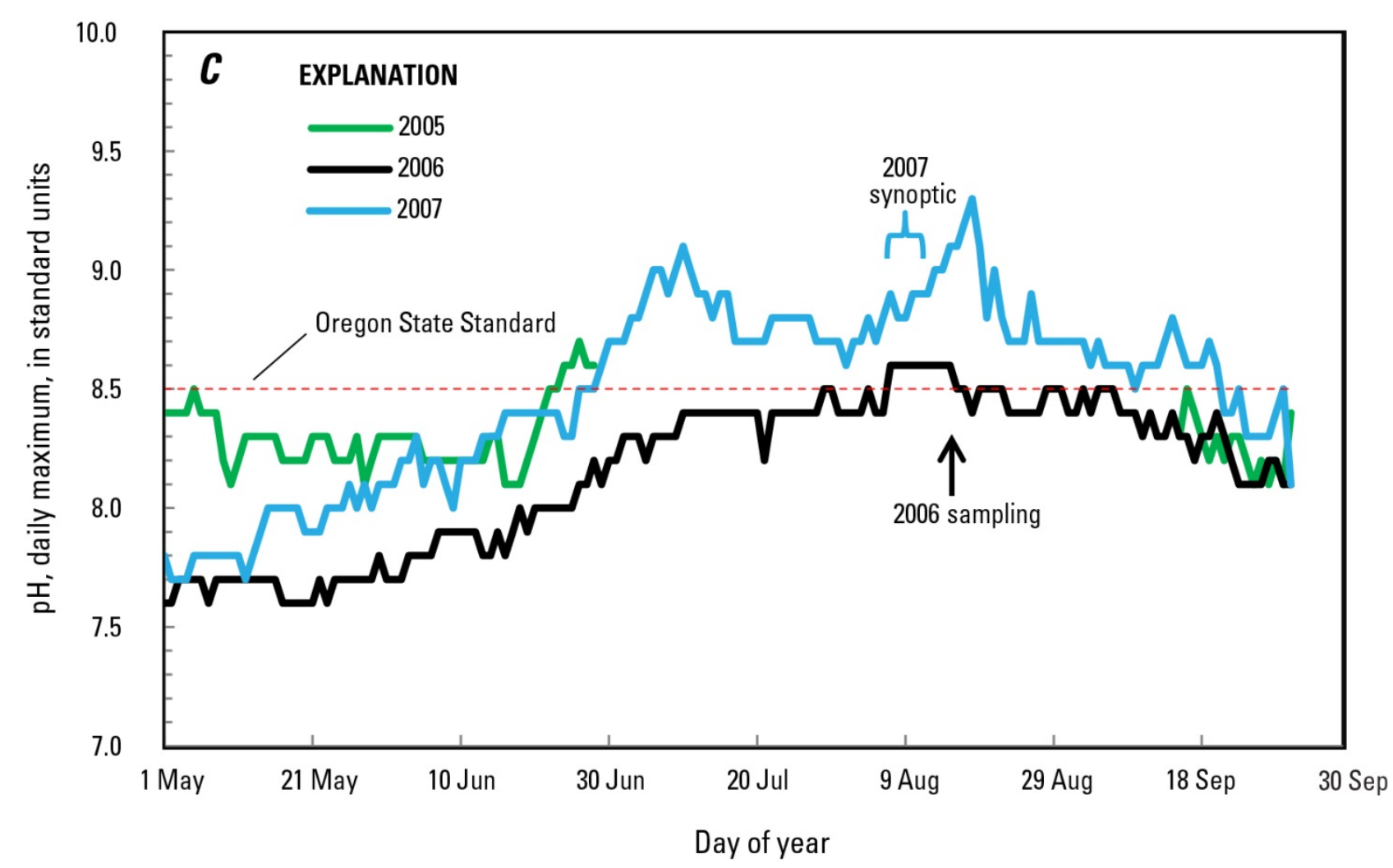

Figure 6-continued. Continuous field parameter data from North Umpqua River near Idleyld Park (14137450), Oregon, 2005-07, showing $(C)$ daily maximum pH. 1995 water temperature data from a monitor located about 0.25 mi downstream of the current location above Rock Creek.

The $16^{\circ} \mathrm{C}$ temperature standard applies when salmon or steelhead are not spawning (June 16-August 31; table 6), and is based on the 7-day running average of the daily maximum water temperatures. This $16^{\circ} \mathrm{C}$ standard was not met on 61-65 (81-84 percent) of the target days during 2005-07. Although 2007 had the highest number of days exceeding the temperature standard, the daily maximum water temperatures averaged $0.5-1{ }^{\circ} \mathrm{C}$ higher in 2005 (a year with relatively low flows) compared with 2006-07. During the 2007 synoptic survey, the maximum temperatures in the Wild and Scenic Reach were $1-1.5^{\circ} \mathrm{C}$ lower than in 1995 and 2005 , but still reached $18.6^{\circ} \mathrm{C}$ (fig. 5). In the summers of 1995 and 2005 , water temperatures reached $21^{\circ} \mathrm{C}$ in the main stem at the downstream end of the study area near the town of Glide (NUGLD).

In all years measured, daily maximum temperatures in most tributaries exceeded $16^{\circ} \mathrm{C}$, and on many days exceeded $20^{\circ} \mathrm{C}$ (fig. 5).
Steamboat Creek and Little River, the largest tributaries to the North Umpqua River, were the warmest and contributed significantly to warming in the mainstem. Similar to the mainstem North Umpqua River, tributary maximum temperatures were consistently less during 2007 than 2005, probably because of a storm and cooler temperatures 2-weeks prior to sampling.

Although most of the data presented here pertain to the non-spawning summer period, the continuous data during the spawning season suggests that temperatures may be problematic for fish in the Wild and Scenic Reach. Data from the Idleyld Park monitor indicate that the $13^{\circ} \mathrm{C}$ spawning criterion, the temperature standard in effect when salmon or steelhead are spawning in the river (September 1-June 15; table 6), was not met on an average $38 \pm 12$ days each year between 2001 and 2012, or about 13-20 percent of the target days (U.S. Geological Survey, 2013). 
Dissolved Oxygen - ODEQ has a tiered system for minimum DO in the study area that sets an absolute threshold, but allows for some variability from the ideal. Table 6 shows the standards for non-spawning periods: $8.0 \mathrm{mg} / \mathrm{L}$ (running average of the 30-day mean minimum), $6.5 \mathrm{mg} / \mathrm{L}$ (running average of the 7-day minimum mean), and an absolute minimum of 6 $\mathrm{mg} / \mathrm{L}$. Although summer is not considered a spawning period for salmonids in the North Umpqua River, these values are the most relevant ODEQ standards for comparison with data from the synoptic surveys.

During the synoptic surveys, DO concentrations in the main stem and the tributaries were never less than $8 \mathrm{mg} / \mathrm{L}$ (fig. 5), which, for the main stem, was confirmed by the continuous monitor near the downstream end of the Wild and Scenic Reach at Idleyld Park (fig. $6 B$ ). Additional data from the BSODA and COPEG monitors (not shown) show DO concentrations greater than $8 \mathrm{mg} / \mathrm{L}$ at those sites during the period of record that began in 2000 . Some sites had diel minimum concentrations of DO less than $8.5 \mathrm{mg} / \mathrm{L}$ (including Little River, Lake Creek, and Steamboat Creek during multiple summers, the North Umpqua River at COPEG in 1995, and NUBWC and NUGLD in 2005), but these were minor decreases that did not violate the DO standards.

The DO concentrations in the Wild and Scenic Reach during the spring-summer algae growth period are affected by reaeration, algal photosynthesis, and water temperature. In summer, DO in the Wild and Scenic Reach rarely is less than $100-115$ percent saturation, with diel variations of 10-13 percent, and the DO largely is in phase with water temperature (fig. 7). The diel maximum DO occurs a few to several hours earlier in the day than the peak water temperature.

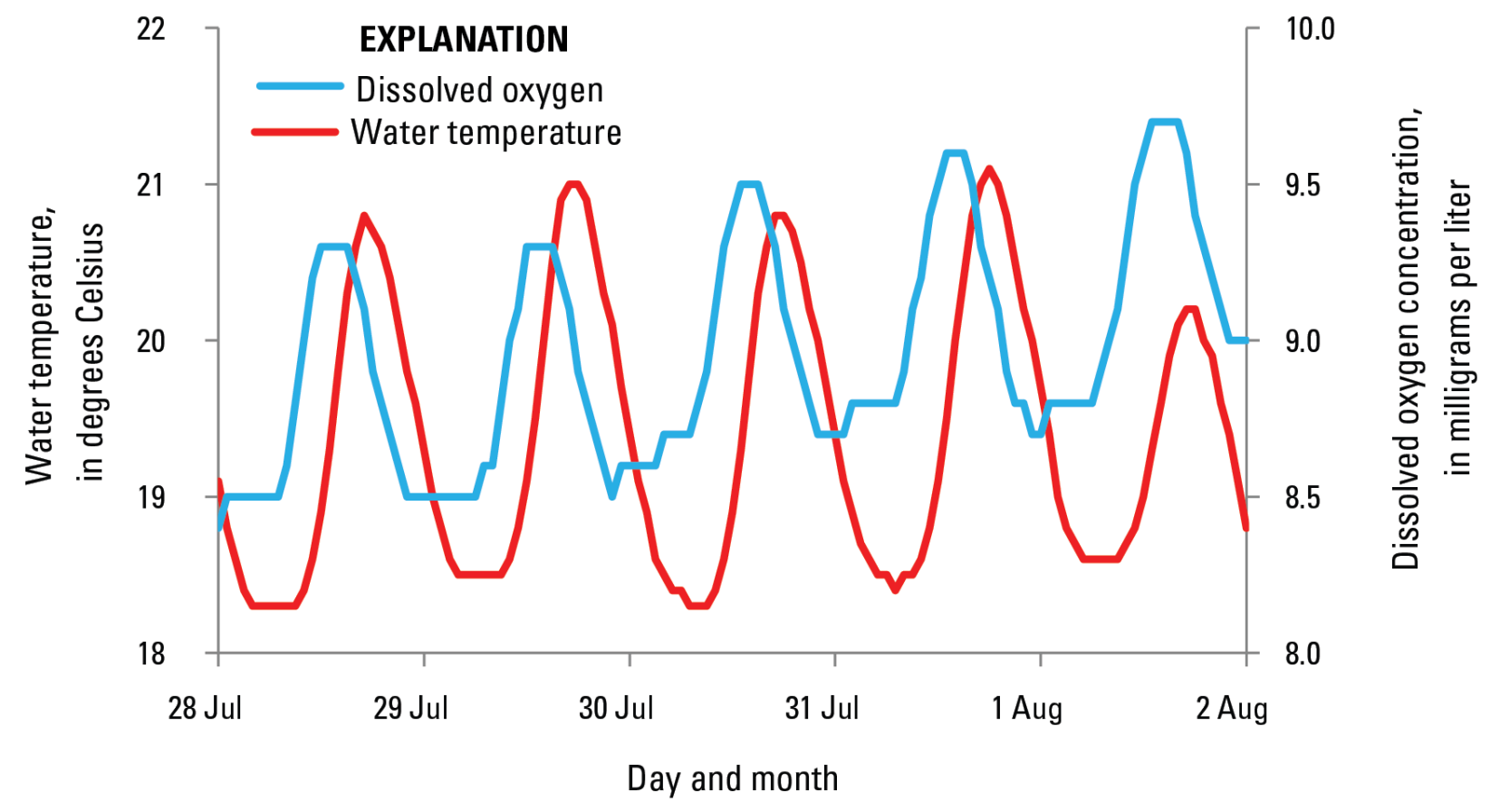

Figure 7. Diel patterns in water temperature and dissolved oxygen in the North Umpqua River near Idleyld Park, July 28-August 2, 2005. 
The data indicate that during summer, photosynthesis exerts moderate control on DO in the North Umpqua River. Photosynthetic DO production outpaces reaeration and solubility during the peak of daytime sunlight. Light wanes rapidly in the mid-afternoon in this steep-walled canyon, reducing photosynthesis despite water temperatures that are still increasing. The combination of reaeration, reduced DO solubility at higher water temperature, and benthic respiration causes DO to decrease. In the Wild and Scenic Reach, where there are numerous riffles and rapids, the photosynthetic effect on DO is muted as turbulent conditions allow DO outgassing to the atmosphere. Turbulence is a moderating factor for DO because it limits diel DO maximums through outgassing, and increases diel DO minimums through aeration.

Steamboat Creek and Little River originate in the Western Cascades and have a lower, longer summer low-flow period, are warmer, and do not reaerate as effectively as the main stem or tributaries in the upper basin. Consequently, these streams can be supersaturated (DO more than 110-133 percent saturation) during summer (appendix table $\mathrm{C} 1$ ), the result of high rates of algal photosynthesis and limited turbulenceinduced loss of DO through air exchange. The direct effect of tributary DO cycles on the main stem is small, however, because of the low percentage of flow contributed during summer.

pH-The $\mathrm{pH}$ data also indicate high rates of algal photosynthesis in the North Umpqua River. Daily maximum $\mathrm{pH}$ was greater than the ODEQ standard of 8.5 units in the downstream reaches at NURCR and NUGLD during the synoptic survey 2007, and was slightly less than 8.5 downstream of Steamboat Creek (NUSTM) in most years (fig. 5). In the main stem downstream of Steamboat Creek, $\mathrm{pH}$ typically increased by approximately $0.8 \mathrm{pH}$ units from morning to afternoon, an effect of algal photosynthesis consuming carbon dioxide $\left(\mathrm{CO}_{2}\right)$ (Wetzel, 1993).

At NURCR, from 2005 to 2007, daily maximum $\mathrm{pH}$ generally exceeded 8.5 units for a period lasting from several weeks as much as $2-$ 3 months. During 2007 (fig. 6C), pH at NURCR exceeded 8.5 units from early July until the end of September, reaching a summer maximum of $9.3 \mathrm{pH}$ units on August 18, 2007, the secondhighest $\mathrm{pH}$ on record at the site. During 2006, $\mathrm{pH}$ was about $0.2-0.4$ units less than in 2007, reaching a maximum of 8.6 units and exceeding 8.5 units for a total of about 2 weeks. Maintenance problems hampered $\mathrm{pH}$ data collection during summer 2005.

Only one site upstream of Soda Springs Reservoir (the main stem upstream of Toketee Lake, at TOKLI) had $\mathrm{pH}$ values as high as 8.5 during the 2007 synoptic survey (fig. 5). Continuous-monitor data from that site (fig. 8) confirm that daytime $\mathrm{pH}$ routinely attained or exceeded $8.5 \mathrm{pH}$ units during the week of the 2007 synoptic survey. The continuous monitor data also show a clear relation between $\mathrm{pH}$ and stream stage: $\mathrm{pH}$ increased sharply around noon each day, when flow was rapidly increased several hundred $\mathrm{ft}^{3} / \mathrm{s}$ for power generation at Lemolo 2 powerhouse. When flow was reduced, $\mathrm{pH}$ dropped rapidly. The forebay and tailrace for the Lemolo 2 powerhouse previously have had nuisance growths of Cladophora that produce high pH (PacifiCorp, 1995; Harza Engineering Co., Inc., 1999), but the high afternoon $\mathrm{pH}$ and low morning DO values $(<8 \mathrm{mg} / \mathrm{L})$ at TOKLKI could also be caused by cycles of photosynthesis and respiration and abundant periphytic growth of Cladophora, Nostoc, and other types of algae in the upstream reach (photograph 8). Engineering changes since 2007 may have reduced the incidence of high $\mathrm{pH}$ at this location (R. Grost, PacifiCorp, Written comm., August 2014). 

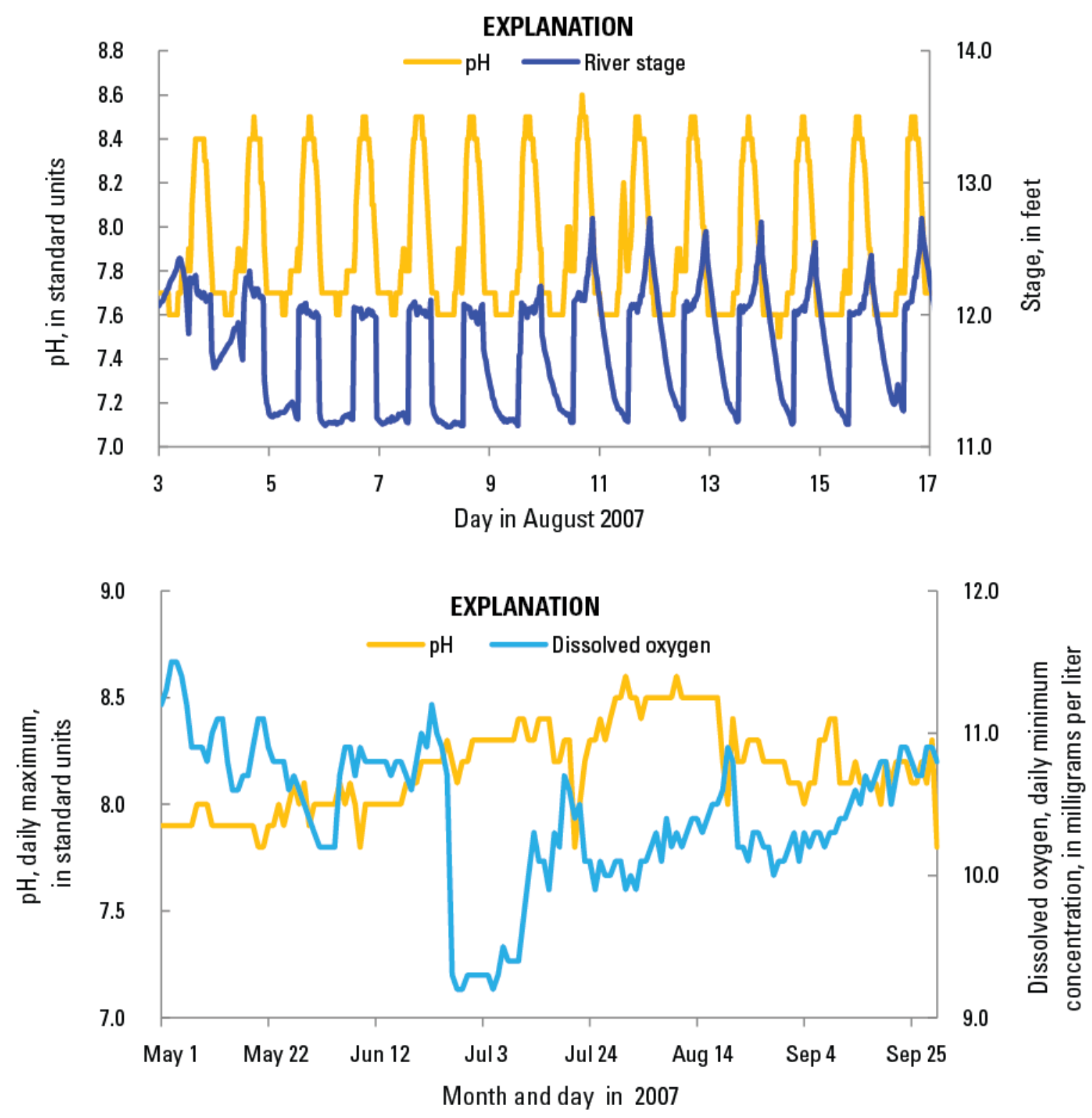

Figure 8. $(A)$ river stage and $\mathrm{pH}$ during the August 2007 synoptic survey and $(B)$ daily maximum $\mathrm{pH}$ and daily minimum dissolved oxygen concentration in the North Umpqua River at the inflow to Toketee Lake (TOKLKI), May-September 2007. 


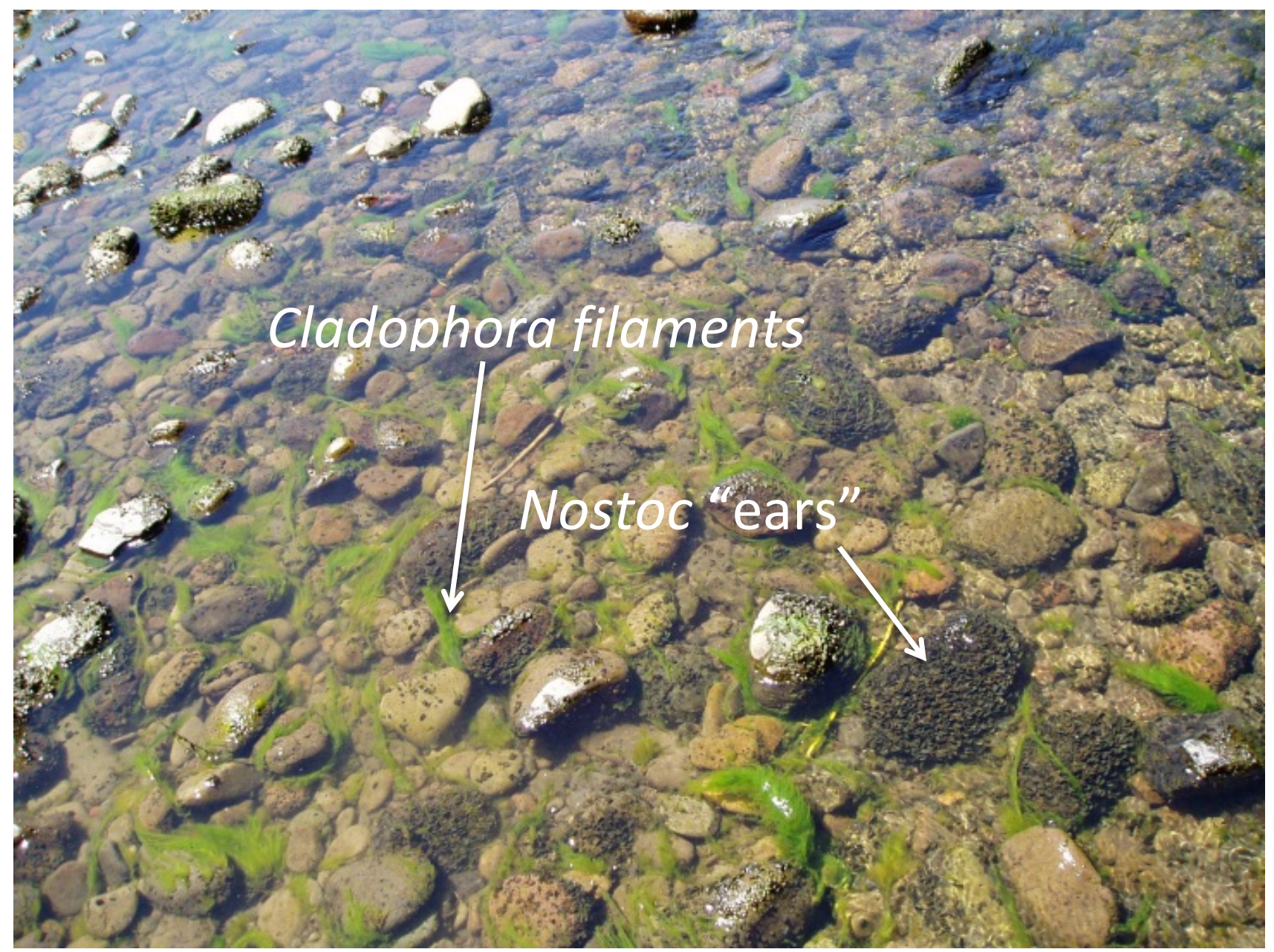

Photograph 8. Filamentous green algae (Cladophora) and "ears" of blue-green algae (Nostoc) at TOKLKI, the inflow to Toketee Lake (photograph by Kurt Carpenter, U.S. Geological Survey, July 26, 2005).

Turbidity-Turbidity is a measure of light scattering resulting from particles suspended in water. Such particles reduce light for photosynthesis and may abrade periphyton from the streambed. Turbidity is lowest in the North Umpqua River during summer; in 2005-07, turbidity was generally less than 1 formazin nephelometric unit (FNU) in July and often less than $0.5 \mathrm{FNU}$ in August. Two notable exceptions occurred at Soda Springs in August 2006 and in July 2007 , when maximum values were as much as 10 and $50 \mathrm{FNU}$, respectively. The 2007 values, which occurred shortly before the synoptic survey that year, resulted from construction at the Soda Springs tailrace. Median daily turbidity values peaked at $13 \mathrm{FNU}$, but decreased to less than $1 \mathrm{FNU}$ and for most of the remainder of the summer. A storm in mid-July 2007 increased streamflow as much as $160 \mathrm{ft}^{3} / \mathrm{s}$ at COPEG and caused a short-term spike in turbidity of about $8 \mathrm{FNU}$. The 2007 synoptic survey was delayed a few weeks to allow the river to clear from these events, but downstream algal growth may have been reduced (light limited) or algal mats may have been abraded by the flow spike and/or elevated turbidity. 


\section{Lake Creek and the Diamond Lake Drawdown}

Given the planned streamflow changes in Lake Creek accompanying the rotenone application in Diamond Lake (fig. 4 and table 2), the study focused on water-quality and algal assemblage changes in Lake Creek and downstream waterways that could be caused either by the altered discharge from Diamond Lake or by a change in the quality of water leaving the lake (Eilers and others, 2007). USGS operated a continuous water-quality and streamflow monitor in Lake Creek downstream from Diamond Lake at Highway 138 (LAKE138) from September 2006 to October 2007. It is difficult to fully evaluate this new record because baseline water-quality data for Lake Creek are lacking.

The variations in Lake Creek conductance (fig. 9) reflect the changing upstream water sources during the course of the restoration project. Source changes help explain differences in water quality, including nutrient and carbon concentrations. Prior to the restoration project, Lake Creek flow was dominated by Diamond Lake water, which had a winter specific conductance of about $30 \mu \mathrm{S} / \mathrm{cm}$ (Eilers, 2008). Flow from Diamond Lake peaked in February 2006, then decreased through spring and summer until flow was halted in August 2006 (fig. 4). Specific conductance increased as discharge decreased, possibly indicating that lake water was becoming more concentrated and (or) groundwater and tributary contributions were increasing.
Streamflow changes in Lake Creek also affected temperature and DO at the LAKE138 monitor. Beginning October 30, 2006, daily mean water temperature increased to about $8{ }^{\circ} \mathrm{C}$ as flow from the lake decreased (fig. 9). Water temperature decreased as flow resumed through the canal on November 7. Simultaneously, DO concentrations decreased by about $4 \mathrm{mg} / \mathrm{L}$ when discharge decreased, then rebounded when flow from the lake resumed. DO concentrations continued to mirror discharge until the Diamond Lake canal was shut off in early March 2007. From then on, DO concentrations decreased through the spring and into the summer, largely the result of increasing water temperatures, as DO saturation remained near 100 percent (data not shown). As water sources changed during this period, $\mathrm{pH}$ decreased slightly, about $0.1-0.2$ units. Daily maximum $\mathrm{pH}$ never exceeded 8.0 units at LAKE138 during spring and summer 2007. 

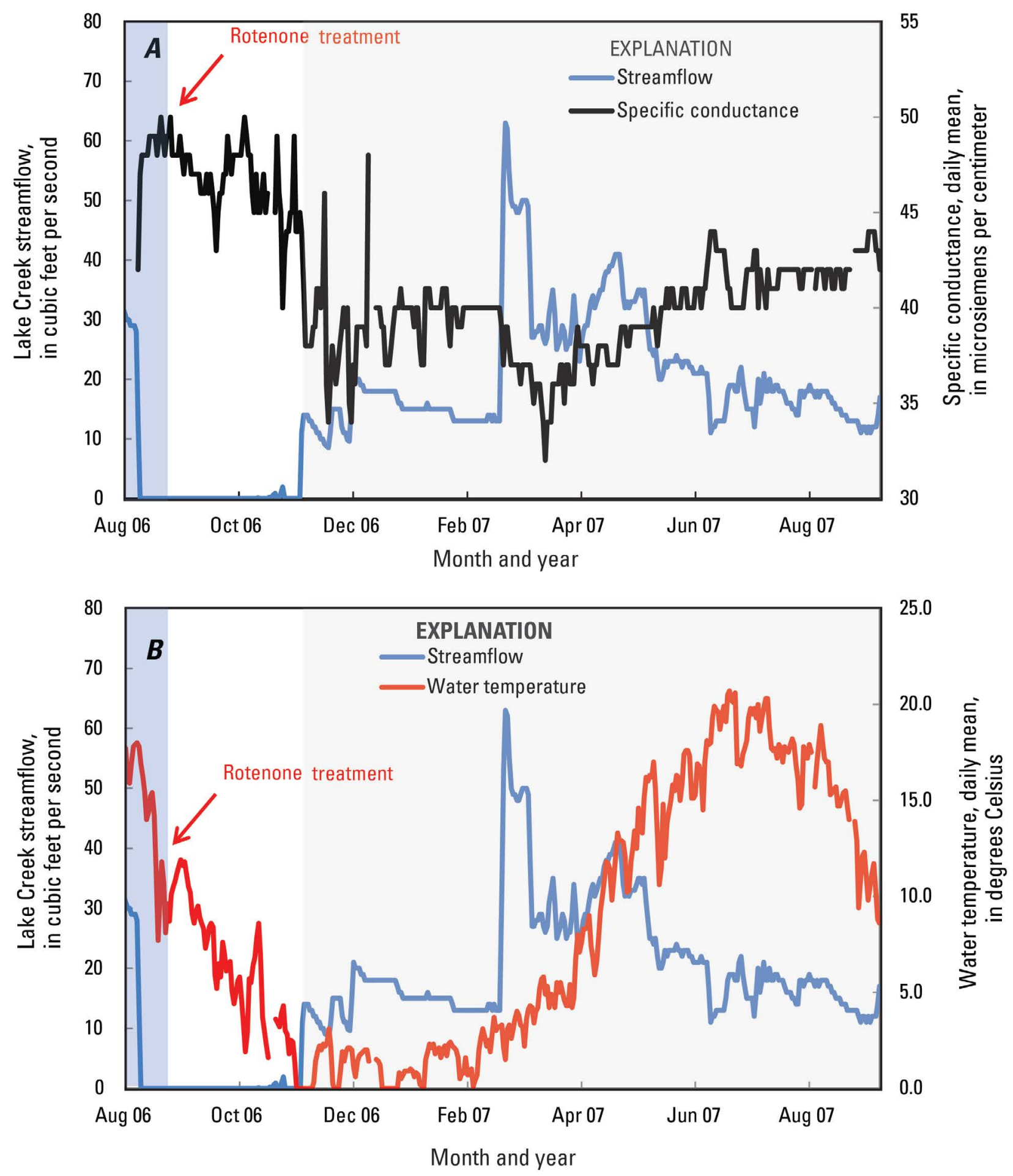

Figure 9. Graphs showing streamflow and $(A)$ daily mean specific conductance and $(B)$ daily mean water temperature, from August 2006 to September 2007. Blue shaded area indicates drawdown period including ceased flow into Lake Creek. Area with no shading indicates the period of the rotenone application until flow resumed in Lake Creek. Gray shaded area indicates post treatment period with minimum flow in Lake Creek. See figure 1 and table 5 for site locations and table 9 for a timeline of events. Figure continues on next page. 

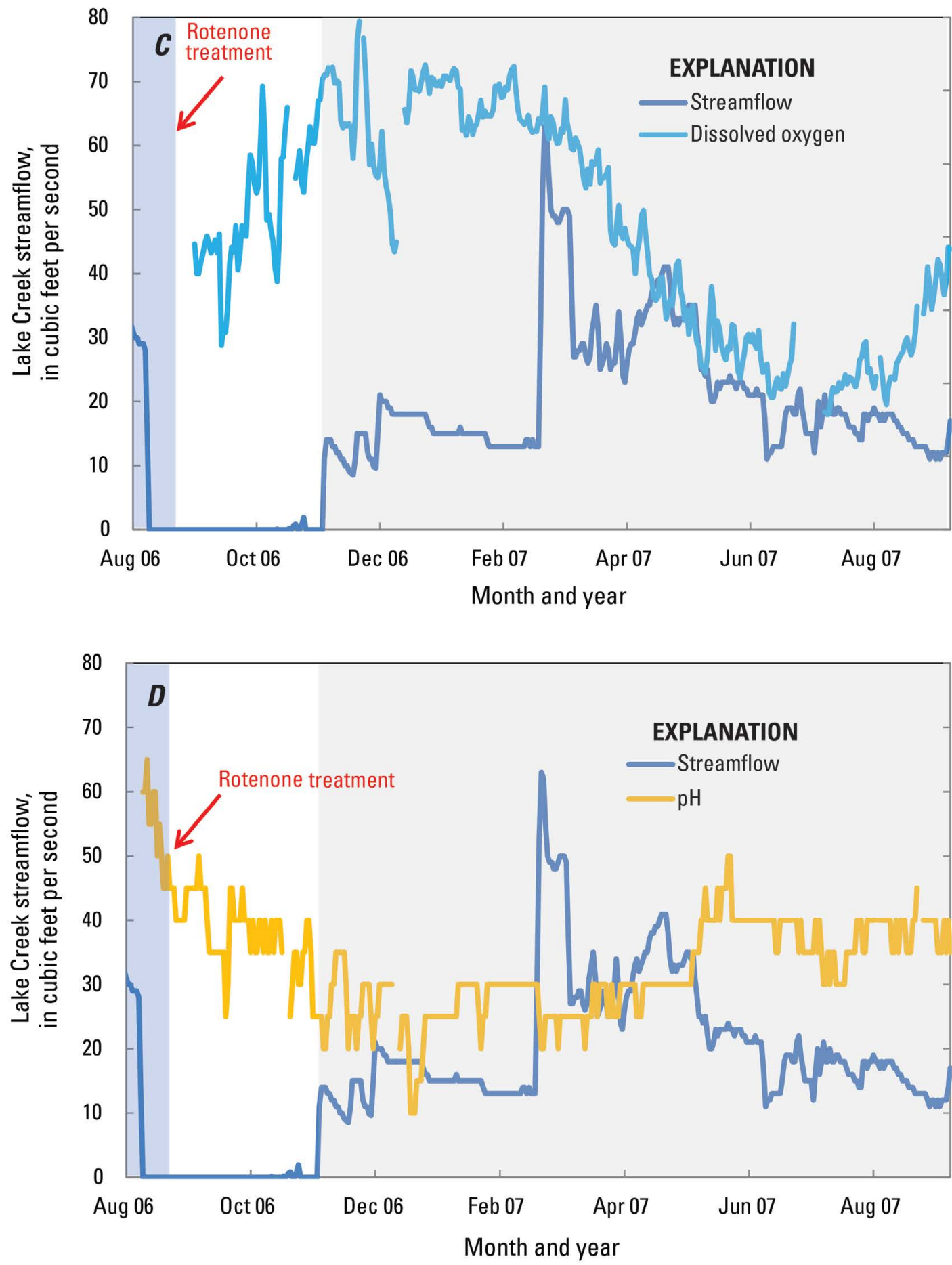

Figure 9-continued. Graphs showing streamflow and $(C)$ daily minimum dissolved oxygen and $(D)$ daily maximum pH in Lake Creek downstream of Diamond Lake at Highway 138 (LAKE138) from August 2006 to September 2007. Blue shaded area indicates drawdown period including ceased flow into Lake Creek. Area with no shading indicates the period of the rotenone application until flow resumed in Lake Creek. Gray shaded area indicates post treatment period with minimum flow in Lake Creek. See figure 1 and table 5 for site locations and table 9 for a timeline of events. 


\section{Nutrients and Organic Carbon}

Data from the synoptic survey in 2005 (Tanner and others, 2006), monthly sampling from May 2006 to September 2007, and the synoptic survey in August 2007 (tables 4 and 5) show that nutrient and organic carbon concentrations were dynamic during the drawdown, rotenone application, and refilling of Diamond Lake. Concentrations of nutrients and organic carbon in Diamond Lake itself were also evaluated in order to estimate export through the canal and Lake Creek during the restoration project. More thorough descriptions of the water-quality and algal conditions in Diamond Lake during this period are available in Eilers (2008), Sytsma and Miller (2008), and Eilers and others (2011).

\section{Diamond Lake, 2006-07}

During the drawdown in 2006, but prior to the rotenone application, Diamond Lake had summer algal blooms and associated poor water quality that typified the previous half-decade (table 1). The Anabaena bloom started in July and continued into August, when the lake was thermally stratified, with a warm epilimnion extending to a depth of about $30 \mathrm{ft}$ (Eilers and others, 2008). Epilimnetic DO was supersaturated and $\mathrm{pH}$ exceeded 9.5 units. The bloom reduced concentrations of biologically available nutrients at the surface, resulting in undetectable concentrations $(<0.005 \mathrm{mg} / \mathrm{L})$ of $\mathrm{NH}_{4}, \mathrm{NO}_{3}$, and SRP. In contrast, total nitrogen (TN) and TP concentrations were $0.2-0.4$ and $0.02-0.04 \mathrm{mg} / \mathrm{L}$, respectively, suggesting that inorganic $\mathrm{N}$ and $\mathrm{P}$ had been incorporated into algal biomass, leaving mostly organic forms in the water column. The hypolimnion, however, was anoxic and contained higher concentrations of $\mathrm{NH}_{4}$ and SRP, with TN and TP concentrations exceeding 0.5 and 0.05 $\mathrm{mg} / \mathrm{L}$, respectively. Turnover in late August mixed the water column, temporarily reducing the bloom, but populations rebounded, and by the end of August, Chl- $a$ had increased to about $20 \mu \mathrm{g} / \mathrm{L}$.

ODFW applied rotenone to Diamond Lake September 14-18, 2006. For the next 2 months, decomposing fish released organic material and nutrients into the water column and increased the 5-day biochemical oxygen demand (Eilers and others, 2011). During October and November 2006, the bloom intensified in response to increases in bio-available nutrients, eventually producing Chl- $a$ concentrations greater than 70 $\mu \mathrm{g} / \mathrm{L}$ and Anabaena cell densities approaching 80,000 cells per $\mathrm{mL}$ (Eilers and others, 2008). The Oregon Health Authority issued a human-health advisory from October 5-November 16, 2006 (42 days), and the lake was closed to water contact recreation (table 1). Surface concentrations of TN, $\mathrm{NH}_{4}$, and TOC remained high through fall and into the following spring, when a diatom bloom reduced nutrient concentrations in the lake (Eilers and others, 2011). Following an early start to the summer growing season in 2007, with clear water and minimal algal blooms, dissolved and total nutrient concentrations increased greatly and stayed high from August through October 2007.

\section{Monthly Sampling in Lake Creek and the North Umpqua River, 2006-07}

Patterns of monthly nitrogen, phosphorus, and carbon concentrations are shown in figures 10-12. Two periods of higher concentrations of $\mathrm{NH}_{4}$, $\mathrm{NO}_{3}$, DOC, and TOC were observed. The first occurred near the end of the 10-month drawdown period (table 2). The increase in concentrations of inorganic nitrogen $\left(\mathrm{NH}_{4}\right.$ and $\left.\mathrm{NO}_{3}\right)$ in Lake Creek was attenuated downstream in the Wild and Scenic Reach (fig. 10). $\mathrm{NH}_{4}$ concentrations in the mainstem were low - at or slightly above the laboratory reporting level of $0.02 \mathrm{mg} / \mathrm{L}$; at these low concentrations, these detections should be viewed with cautiously. Nevertheless, these detections of $\mathrm{NH}_{4}$ during summer in the Wild and Scenic Reach are noteworthy in this otherwise nitrogen-depleted river. The consistency of the detections in every sample from late June to midOctober 2006 (fig. 10) suggests that $\mathrm{NH}_{4}$ was present in the water column, and represents a potentially important shift in nitrogen availability from 1995 (Anderson and Carpenter, 1998). 


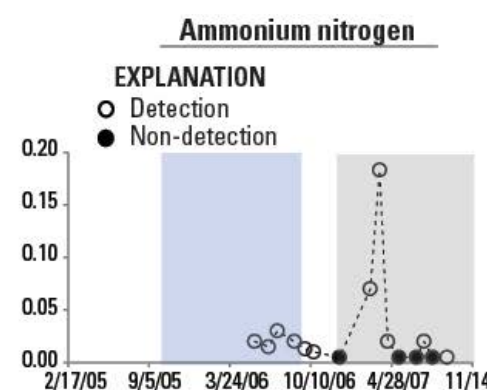

Nitrite plus nitrate nitrogen

Dissolved inorganic nitrogen

Total Kjeldahl nitrogen
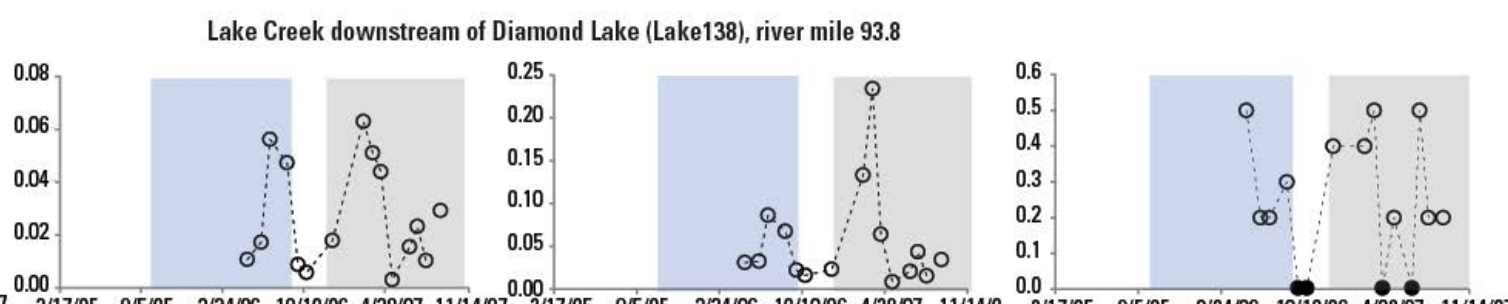

North Umpqua River at the inflow to Toketee Lake (TOKLKI), river mile 77.1
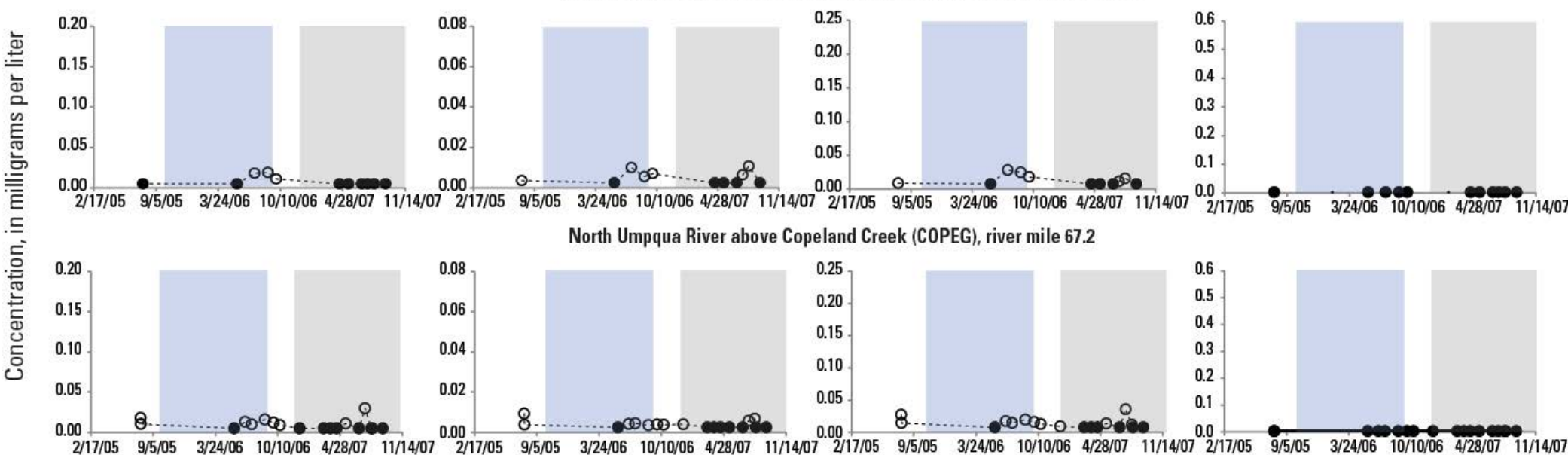

North Umpqua River above Copeland Creek (COPEG), river mile 67.2
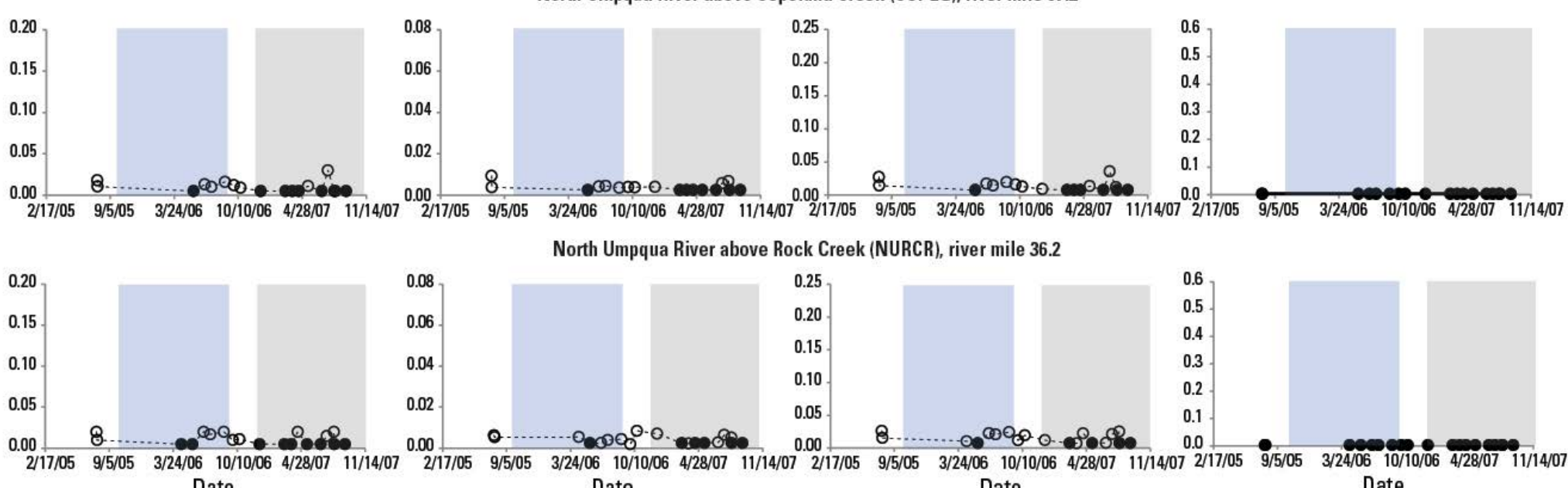

North Umpqua River above Rock Creek (NURCR), river mile 36.2

Figure 10. Monthly nitrogen concentrations in Lake Creek and the downstream North Umpqua River during the Diamond Lake restoration project, 2005-07. Blue shaded area indicates draw-down period. Area with no shading indicates the period of the rotenone application until flow resumed in Lake Creek. Gray shaded area indicates post-treatment period with resumed flow in Lake Creek. See figure 1 and table 5 for site locations. Dissolved inorganic nitrogen includes nitrite, nitrate, and ammonium. Detection limits listed in Appendix A. 
Soluble Reactive Phosphorus

Total Phosphorus

EXPLANATION

O Detection

- Non-detection Lake Creek dowstream of Diamond Lake (LAKE138), river mile 93.8
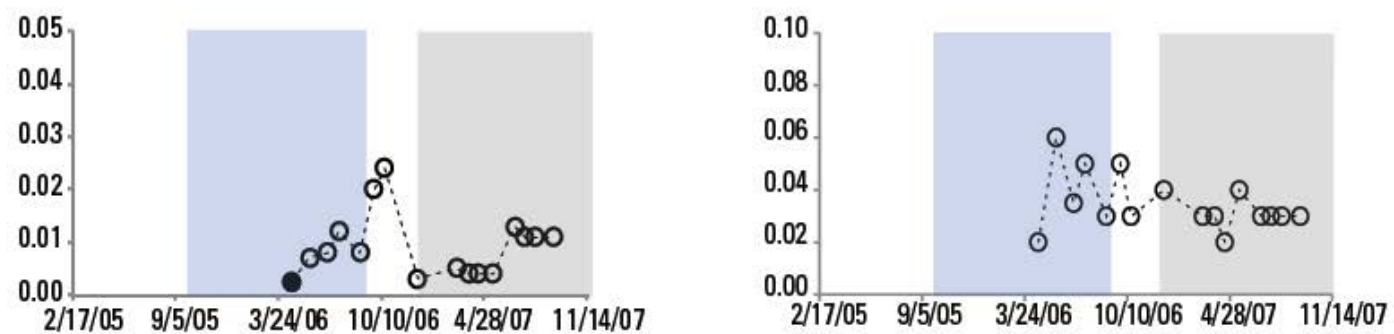

North Umpqua River at Toketee Lake Inflow (TOKLKI), river mile 77.1
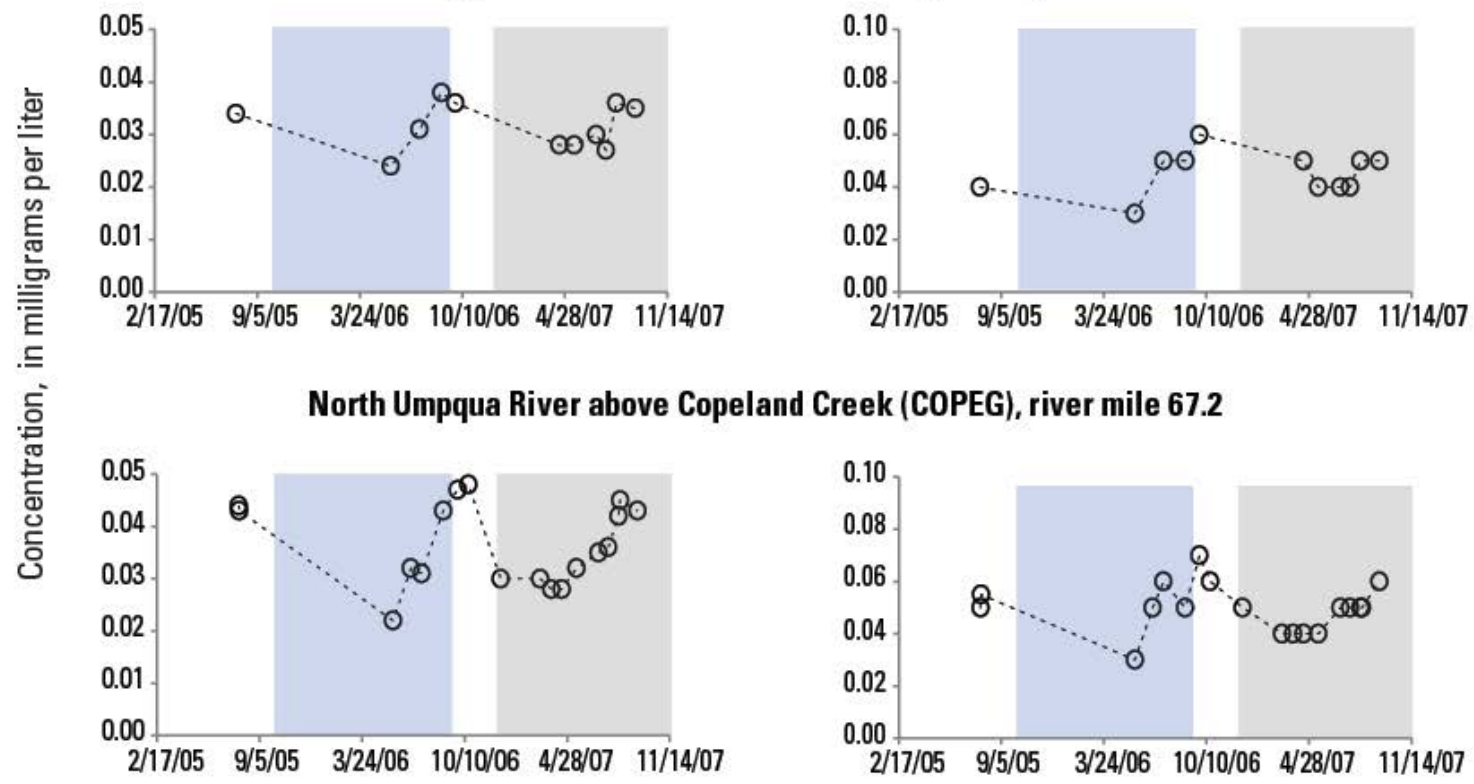

North Umpqua River above above Rock Creek (NURCR), river mile 36.2
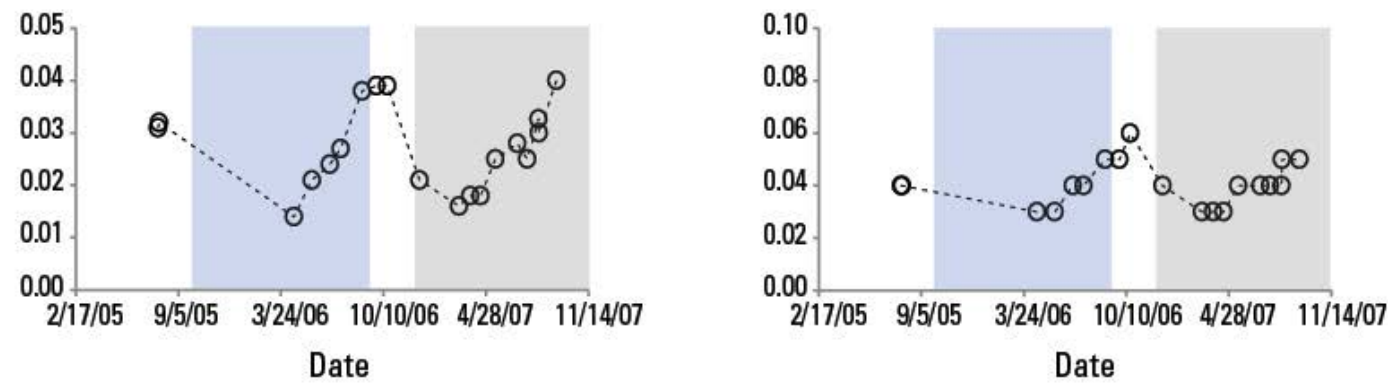

Figure 11. Monthly phosphorus concentrations in Lake Creek and the downstream North Umpqua River during the Diamond Lake restoration project, 2005-07. Blue shaded area indicates drawdown period. Area with no shading indicates the period of the rotenone application until flow resumed in Lake Creek. Gray shaded area indicates post-treatment period with resumed flow in Lake Creek. See figure 1 and table 5 for site locations. Dissolved inorganic nitrogen includes nitrite, nitrate, and ammonium. Detection limits listed in Appendix A. 


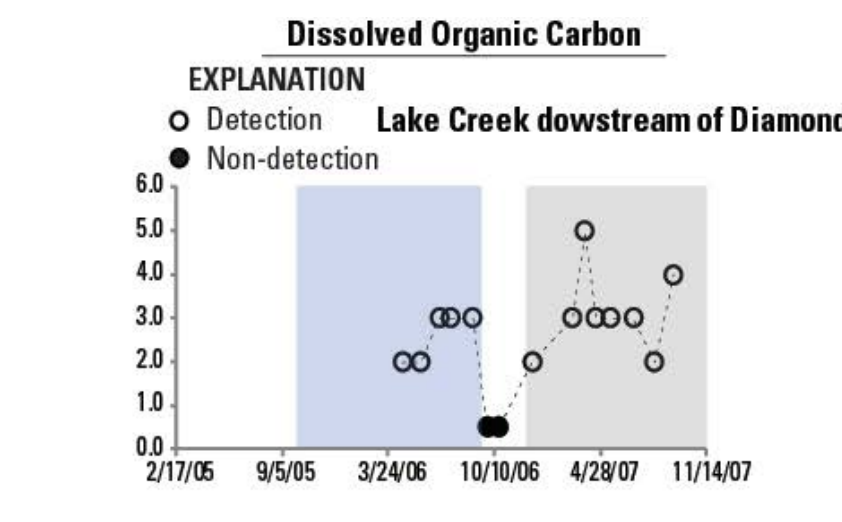

Total Carbon

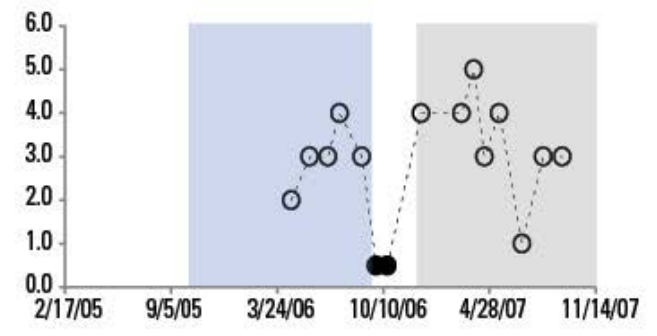

North Umpqua River at Toketee Lake Inflow (TOKLKI), river mile 77.1
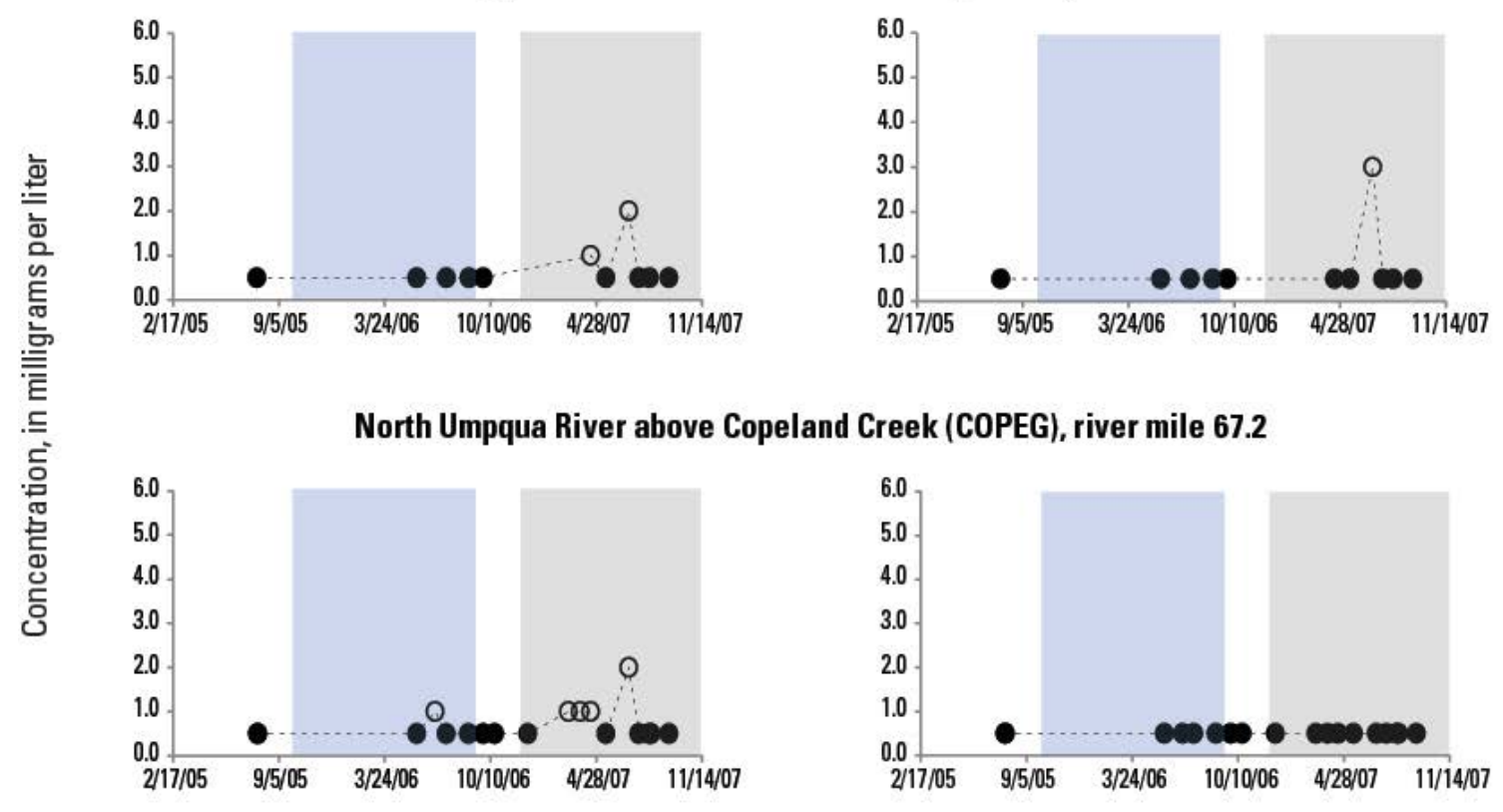

North Umpqua River above above Rock Creek (NURCR), river mile 36.2
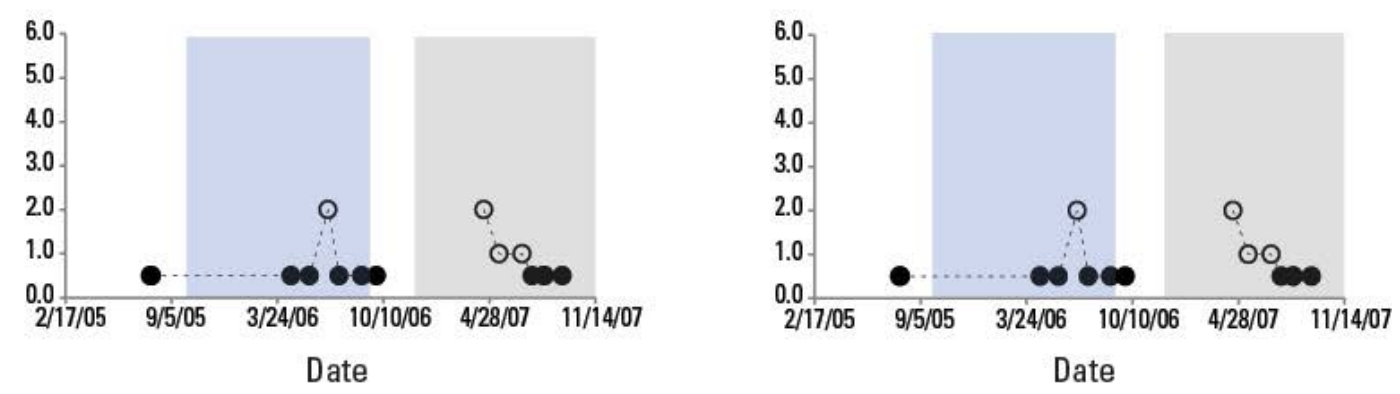

Figure 12. Monthly carbon concentrations in Lake Creek and the downstream North Umpqua River during the Diamond Lake restoration project, 2005-07. Blue shaded area indicates drawdown period. Area with no shading indicates the period of the rotenone application until flow resumed in Lake Creek. Gray shaded area indicates posttreatment period with resumed flow in Lake Creek. See figure 1 and table 5 for site locations. Dissolved inorganic nitrogen includes nitrite, nitrate, and ammonium. Detection limits listed in Appendix A. 
Although elevated TKN concentrations were measured in Lake Creek, TKN was not detected downstream at any of the other monthly sampling sites (fig. 10). Because Diamond Lake epilimnetic concentrations of TN and TP were high and dissolved nutrients were low during the drawdown (Eilers and others, 2011), the detected $\mathrm{NH}_{4}$ and $\mathrm{NO}_{3}$ in Lake Creek likely resulted from mineralization of organic nitrogen leaving Diamond Lake and the subsequent nitrification of $\mathrm{NH}_{4}$ to $\mathrm{NO}_{3}$; however, data to confirm these transformations are unavailable. Carbon concentrations also were much higher in Lake Creek toward the end of the drawdown period in June-August: $3 \mathrm{mg} / \mathrm{L}$ DOC and 2-4 mg/L TOC. Carbon concentrations decreased to less than 0.05 $\mathrm{mg} / \mathrm{L}$, the laboratory detection limit, after the drawdown ended and flows from Diamond Lake stopped, suggesting that the drawdown of the lake was the source of the high carbon concentrations.

The elevated concentrations of $\mathrm{NO}_{3}$ in Lake Creek during the drawdown were attenuated downstream (fig. 10), but consistent detections of $\mathrm{NO}_{3}$ in summer 2006 and again in summer 2007 corresponded to water releases from Diamond Lake (Eilers, 2008), although concentrations were relatively low $(<0.01 \mathrm{mg} / \mathrm{L})$.

Phosphorus exported from Diamond Lake during the drawdown was primarily in particulate form, likely algal cells and other organic particles. TP concentrations peaked at $0.06 \mathrm{mg} / \mathrm{L}$ in Lake Creek on May 24 (fig. 11), when SRP concentration was below $0.01 \mathrm{mg} / \mathrm{L}$. The resulting SRP:TP ratio of about 0.12 (fig. 13) indicates that almost 90 percent of the phosphorus was in particulate form. SRP in Lake Creek increased slightly toward the end of the drawdown period, and increased again to 0.024 $\mathrm{mg} / \mathrm{L}$ (fig. 11) when flows from Diamond Lake ended and groundwater and tributaries became dominant. Downstream in the Wild and Scenic Reach, phosphorus concentrations were much higher than in Lake Creek owing to the input of groundwater-dominated tributaries such as the Clearwater River, which typically contributes $0.07-0.08 \mathrm{mg} / \mathrm{L} \mathrm{SRP.} \mathrm{In} \mathrm{the} \mathrm{rest} \mathrm{of} \mathrm{the} \mathrm{river}$ system, including the Wild and Scenic Reach,
SRP:TP ratios were $0.5-0.75$, indicating that most of the P was dissolved and suggesting that groundwater remained the predominant source of phosphorus.

A larger pulse of $\mathrm{NH}_{4}$ and carbon occurred in Lake Creek in early March 2007 and lasted for at least a few weeks. This pulse started about the time when the canal was closed and flows resumed from Diamond Lake through the natural outlet (fig. 4). During that period, $\mathrm{NH}_{4}$ concentrations in Lake Creek-0.07 and 0.18 $\mathrm{mg} / \mathrm{L}$ on March 6 and 29, 2007, respectivelywere much higher than they were during the drawdown period. DOC and TOC concentrations also were higher at this time (fig. 12). This pulse is likely attributable to the transport of organic matter and fish decomposition residuals from the rotenone treatment in Diamond Lake, although normal decomposition of bottom sediments during the winter also could have contributed. The lack of baseline winter and spring data in Lake Creek or Diamond Lake prevents definitive conclusions about the source of these materials.

Ratios of biologically available forms of dissolved inorganic $\mathrm{N}$ and $\mathrm{P}$ (DIN:SRP) are common indicators of nutrient limitation, along with seasonal and longitudinal patterns in nutrient concentrations. With the notable exception during the drawdown and post-treatment periods in Lake Creek, DIN:SRP ratios were less than 1-2 (fig. 13), indicating potentially strong $\mathrm{N}$ limitation. The DIN:SRP ratio in Lake Creek during the Diamond Lake drawdown in summer 2006 fluctuated between 4 and 10, indicating that Lake Creek was neither strongly N nor P limited. During the post-treatment period, the DIN:SRP ratio in Lake Creek increased substantially to 58 on March 29 as flow from Diamond Lake resumed, indicating an abundance of nitrogen relative to phosphorus. Given the generally ample concentrations of both $\mathrm{N}$ and $\mathrm{P}$, it is likely that neither nutrient was limiting periphyton growth in Lake Creek at that time. 
SRP:TP Ratio

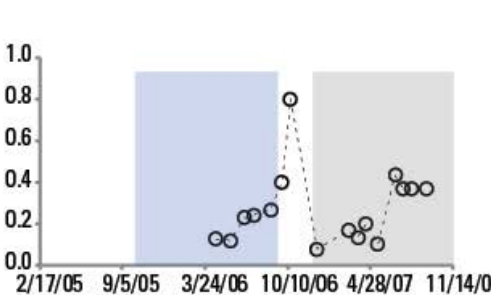

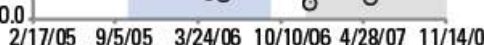

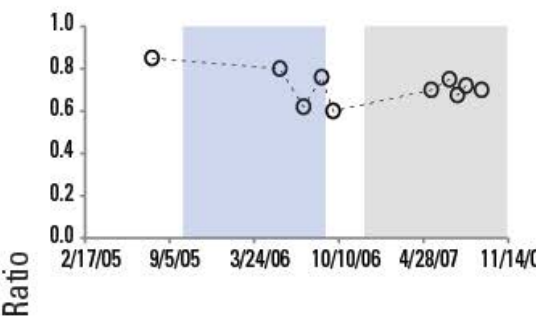

DIN:SRP Ratio

TN:TP Ratio

DOC:TOC Ratio

Lake Creek dowstream of Diamond Lake (LAKE138), river mile 93.8
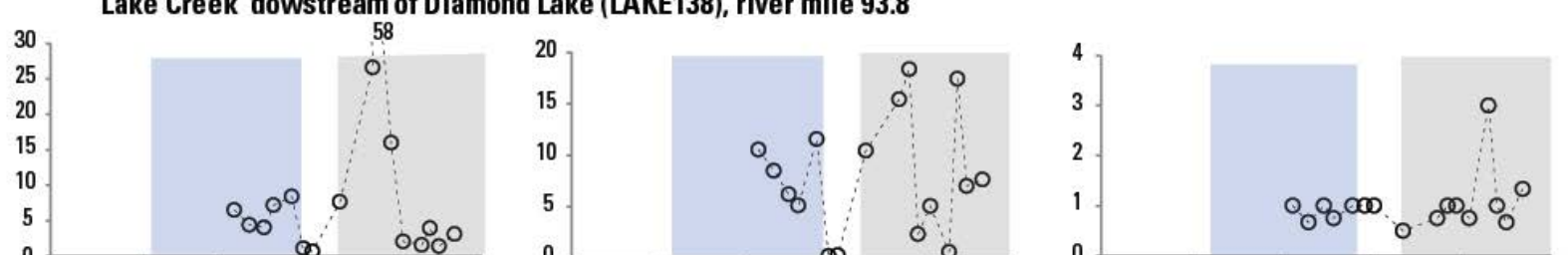

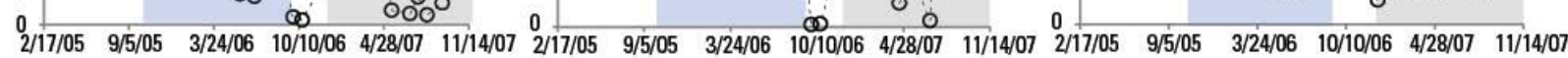

North Umpqua River at Toketee Lake Inflow (TOKLKI), river mile 77.1

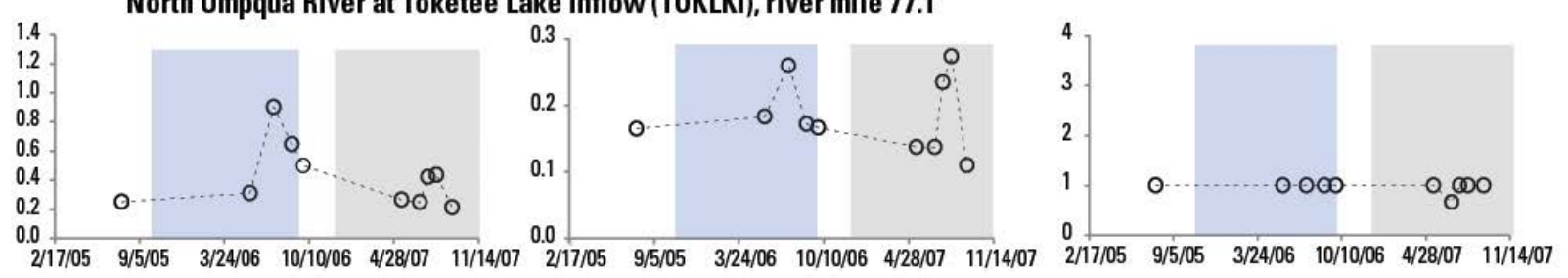

North Umpqua River above Copeland Creek (COPEG), river mile 67.2
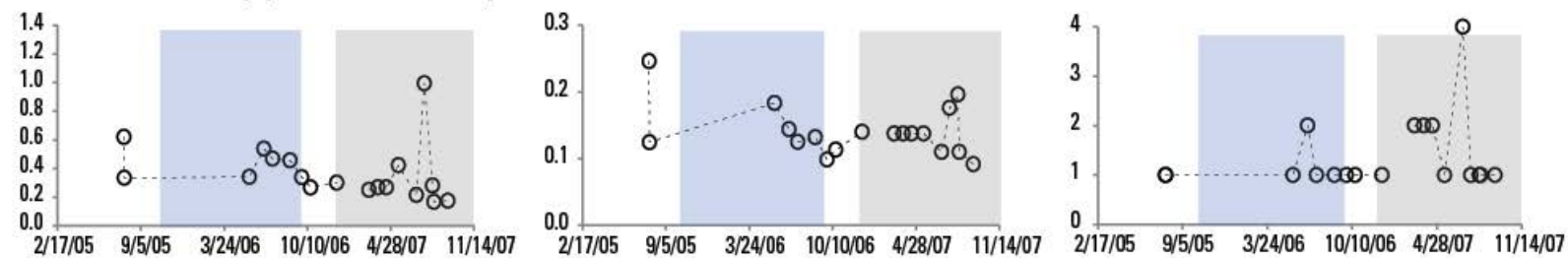

North Umpqua River above above Rock Creek (NURCR), river mile 36.2
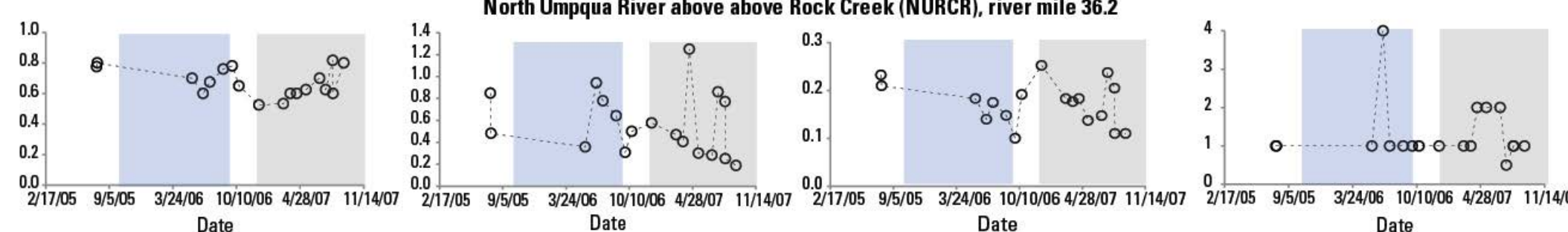

Date

Date

Date

Date

Figure 13. Nutrient and carbon ratios for sites sampled monthly in the North Umpqua River basin during the Diamond Lake restoration project, 2005-07. Blue shaded area indicates drawdown period. Area with no shading indicates the period of the rotenone application until flow resumed in Lake Creek. Gray shaded area indicates post-treatment period with resumed flow in Lake Creek. SRP, soluble reactive phosphorus; TP, total phosphorus; DIN, dissolved inorganic nitrogen; TN, total nitrogen; DOC, dissolved organic carbon; TOC, total organic carbon. See figure 1 and table 5 for site locations. Dissolved inorganic nitrogen includes nitrite, nitrate, and ammonium. 
In contrast to phosphorus, most of the organic carbon exported from Diamond Lake was in dissolved form, with DOC concentrations generally equivalent to TOC concentrations. During the Diamond Lake drawdown in 2006, Lake Creek DOC and TOC increased to 3-5 $\mathrm{mg} / \mathrm{L}$ at the same time the $\mathrm{NH}_{4}$ was increasing. Following the rotenone treatment, there were smaller pulses of DOC and TOC at the downstream sites, particularly at COPEG and NURCR (fig. 12). Several samples from COPEG and NURCR (appendix table $\mathrm{C} 2$ ) had DOC concentrations higher than their respective TOC concentrations, a theoretically impossible result that Aiken and others (2002) attribute to incomplete digestion of certain substances in the TOC analysis. In this case, the DOC analysis is assumed to be more precise and accurate. Overall, these results support the conclusion that most of the organic carbon in Lake Creek was in dissolved form.

\section{Comparison of Nutrient Concentrations and Algal Biomass to Suggested Criteria}

Maximum concentrations of nitrite-plusnitrate $\left(\mathrm{NO}_{2}+\mathrm{NO}_{3}\right)$ were higher than the EPAproposed reference value of $0.005 \mathrm{mg} / \mathrm{L}$ in both the Wild and Scenic Reach and tributaries in 2005 and 2007 (table 7). In contrast, $\mathrm{NO}_{3}$ was not detected in the Wild and Scenic Reach in 1995, although the method of analyses differed (see Methods section). Maximum tributary DIN values were greater than the EPA reference conditions in 1995, 2005, and 2007, and DIN in Lake Creek generally exceeded the reference concentration. TKN concentrations were less than the EPA reference value of $0.05 \mathrm{mg} / \mathrm{L}$ at all sites in 1995 and 2007, but exceeded $0.05 \mathrm{mg} / \mathrm{L}$ at three Wild and Scenic Reach sites in 2005 (NUSTM, NURCR, and NUGLD), potentially reflecting increased periphyton growth in a downstream direction with associated sloughing of algal particles into the water column.

Although tributary concentrations of TP were lower than the EPA reference concentration of $0.009 \mathrm{mg} / \mathrm{L}$ in some tributaries entering the Wild and Scenic Reach (in 1995), all samples collected from the main stem exceeded $0.009 \mathrm{mg} / \mathrm{L}$ in all years (appendix table C1). Maximum SRP values in the Wild and Scenic Reach and tributaries exceeded the suggested criteria to prevent nuisance algal growth in all years.

The prevalence of high phosphorus concentrations from geologic sources in the North Umpqua River and other Cascade Range rivers illustrates the potential shortcomings of the EPA reference condition approach. The EPA uses probabilistic methods to set reference conditions at the $25^{\text {th }}$ percentile of measured streams on an ecoregion basis, but does not consider local geologic influences. Similar local conditions also may apply to EPA reference conditions for DIN and TKN. Reference condition comparisons, therefore, should be taken as only one point of comparison in multifaceted analyses such as this study. Nonetheless, this comparison shows that periphyton growth can be sensitive to nutrient inputs in relatively pristine environments such as the Wild and Scenic North Umpqua River, and that higher concentrations observed between 1995 to 2005-07 could have been sufficient to cause increases in algal growth, biomass accumulation, and primary productivity.

\section{Changes in Water-Quality Conditions between 1995 and 2005-07}

In 2005, the average specific conductance at sites in the Wild and Scenic Reach was 11 percent higher than in 1995 (table 10). Nutrient concentrations in the Wild and Scenic Reach were seemingly higher in 2005, although some of the percentages represent only small changes in concentrations, which may not be significant, especially for $\mathrm{NO}_{3}$ and $\mathrm{NH}_{4}$. For example, although table 10 shows that $\mathrm{NO}_{3}$ concentrations in the Wild and Scenic Reach were 50\% higher in 2005 compared with 1995, this was based on substituting one-half the laboratory reporting level for non-detections (see Methods section). If, however, the reporting level $(0.005 \mathrm{mg} / \mathrm{L})$ were used for non-detections, then the change in $\mathrm{NO}_{3}$ concentrations is just 2 percent. 
TP concentrations from the six main-stem sites in the Wild and Scenic Reach were higher in 2007 than 1995 (fig. 14). Average TP in the 2005 synoptic samplings was 35 percent higher (table 10 ), and in 2007, 54 percent higher than 1995, with both changes being significant $(\mathrm{P}<0.05)$. SRP concentrations in the Wild and Scenic Reach were 33 percent higher in 2005 than 1995, with little change in concentrations between 2005 and 2007; tributary concentrations were 13 percent less in 2007 (table 10).

Although TP concentrations were higher in the main stem in 2005-07 than 1995, the spatial distribution was similar among years (fig. 14). The Clearwater River (CLRAS) at RM 75.4 is one of the largest sources of $\mathrm{P}$ because it contributes a consistent $0.07-0.08 \mathrm{mg} / \mathrm{L}$ from relatively high amounts of spring-fed discharge. Each year, main-stem TP concentrations decreased with increasing distance downstream of the hydroelectric project at Soda Springs Reservoir, likely a function of in-stream metabolism, including nutrient uptake by periphyton.

In 1995 and 2007, TP concentrations increased slightly between Apple Creek (RM 57.5) and Mott Bridge (RM 53.2), probably attributable to Panther Creek, which had the highest TP concentrations of all the small tributaries upstream of Steamboat Creek. Steamboat Creek had lower TP concentrations than the main stem and enough flow to dilute $\mathrm{P}$ concentrations at the next downstream site, although nutrient uptake by periphyton likely also contributed to the decrease. Farther downstream, TP concentrations continued to decrease despite inflow from Rock Creek, which was another significant source of TP.

$\mathrm{NH}_{4}$ concentrations in the Wild and Scenic Reach were apparently higher in 2005 compared with 1995, when no detections occurred (table 10). In 2005, two low-level $\mathrm{NH}_{4}$ detections of $0.02-0.03 \mathrm{mg} / \mathrm{L}$ at NURCR and NUGLD resulted, statistically, in a 50 percent increase over 1995. These low-concentration detections are at or just above the laboratory reporting limit of $0.02 \mathrm{mg} / \mathrm{L}$, and should be viewed cautiously.

$\mathrm{NO}_{3}$ concentrations in the main-stem Wild and Scenic Reach were essentially indistinguishable between 1995 and 2005, with all values near or below the laboratory reporting level of $0.005 \mathrm{mg} / \mathrm{L}$ in 2005 (appendix table C1). Mainstem $\mathrm{NO}_{3}$ concentrations in this reach were slightly higher in 2007. Tributary concentrations of $\mathrm{NO}_{3}$ were much higher in 2005-07 compared with 1995, at concentrations well above reporting levels and unlikely to be related to any potential laboratory differences. The higher $\mathrm{NO}_{3}$ values from these tributaries and from upstream reservoirs provide logical sources for the $\mathrm{NO}_{3}$ observed in the Wild and Scenic Reach.

Given the cyanobacterial blooms and likely associated N-fixation in Diamond and Lemolo Lakes since the late 1990s, it is plausible that production and downstream transport of inorganic $\mathrm{N}$ could be occurring. Two sites downstream from Diamond and Lemolo Lakes (LAKEM and LEM1T/G), had substantially higher $\mathrm{NO}_{3}$ values in 2005 compared with 1995 (appendix table C1), possibly reflecting greater nitrogen export from those lakes resulting from algal blooms. $\mathrm{NO}_{3}$ concentrations in the bypass reach downstream of Lemolo Lake (LEM1T/G) were much higher in 2005 and 2007 (0.026-0.027 mg/L) compared with $<0.005 \mathrm{mg} / \mathrm{L}$ in 1995 .

The higher $\mathrm{NO}_{3}$ concentrations at LAKEM and LEM1T/G in 2005, when combined with similar observations from the tributaries, provide further evidence that $\mathrm{NO}_{3}$ loading to the Wild and Scenic Reach was greater in 2005 compared with 1995.

In 1995, TKN was not detected at any of the 6 mainstem sites in the Wild and Scenic Reach, but in 2005, concentrations at NUSTM and NURCR were $0.07 \mathrm{mg} / \mathrm{L}$ (appendix table C1). It is not known whether this organic material was algal, but possible sources include phytoplankton export from Diamond and Lemolo Lakes, and sloughing of periphyton. 
A. Mainstem North Umpqua River

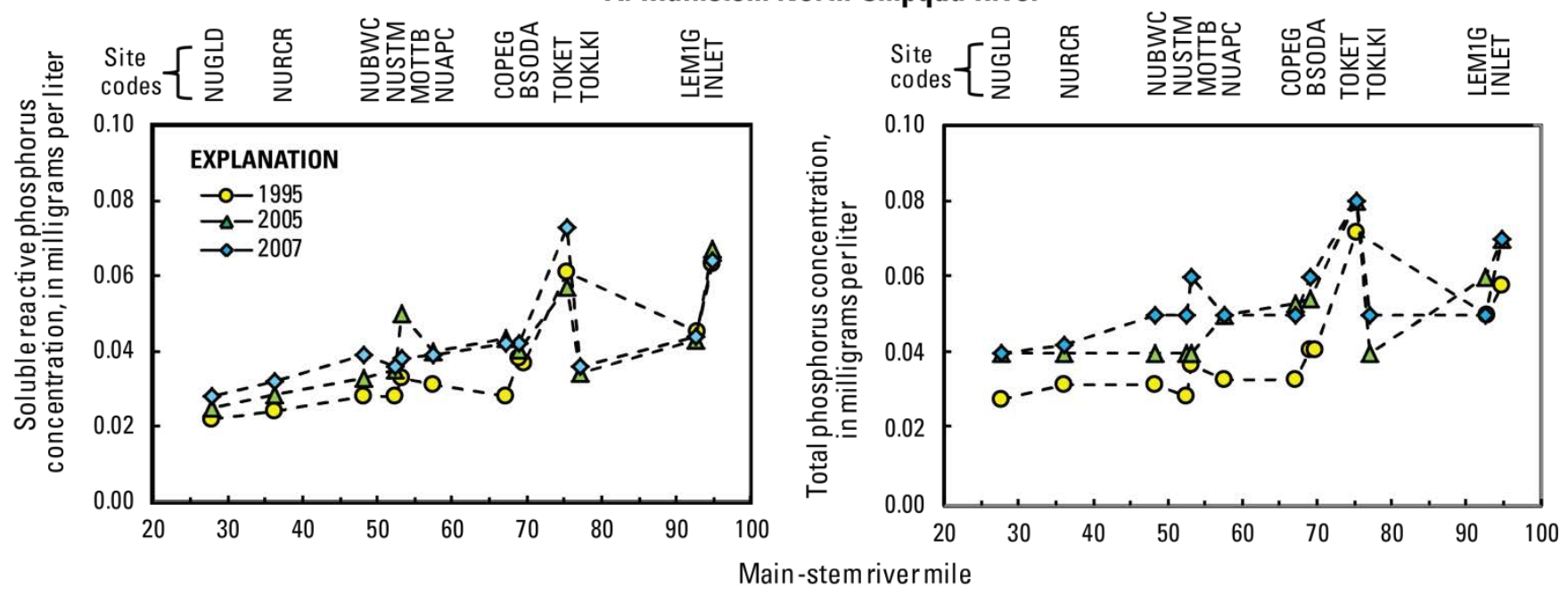

\section{B. Tributaries}

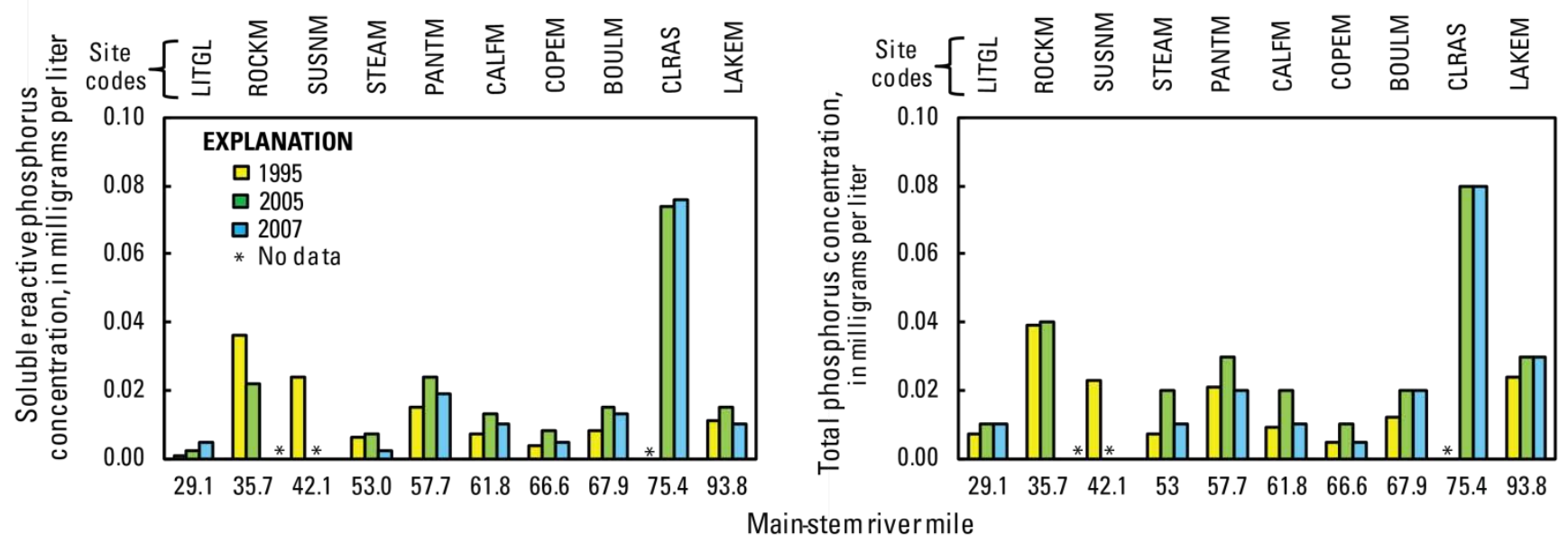

Figure 14. Concentrations of soluble reactive phosphorus and total phosphorus in the $(A)$ main-stem North Umpqua River and (B) tributaries during the 1995, 2005, and 2007 synoptic surveys. Site codes defined in table 5. 
A. Mainstem North Umpqua River

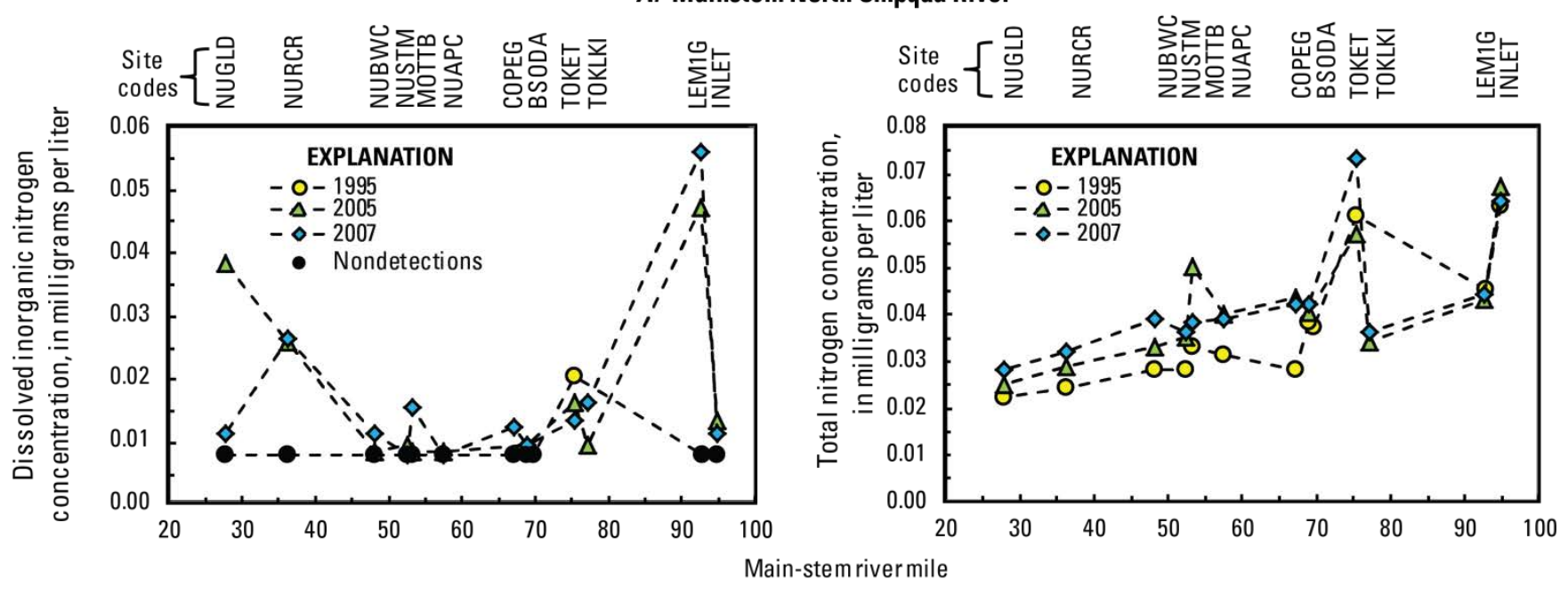

B. Tributaries
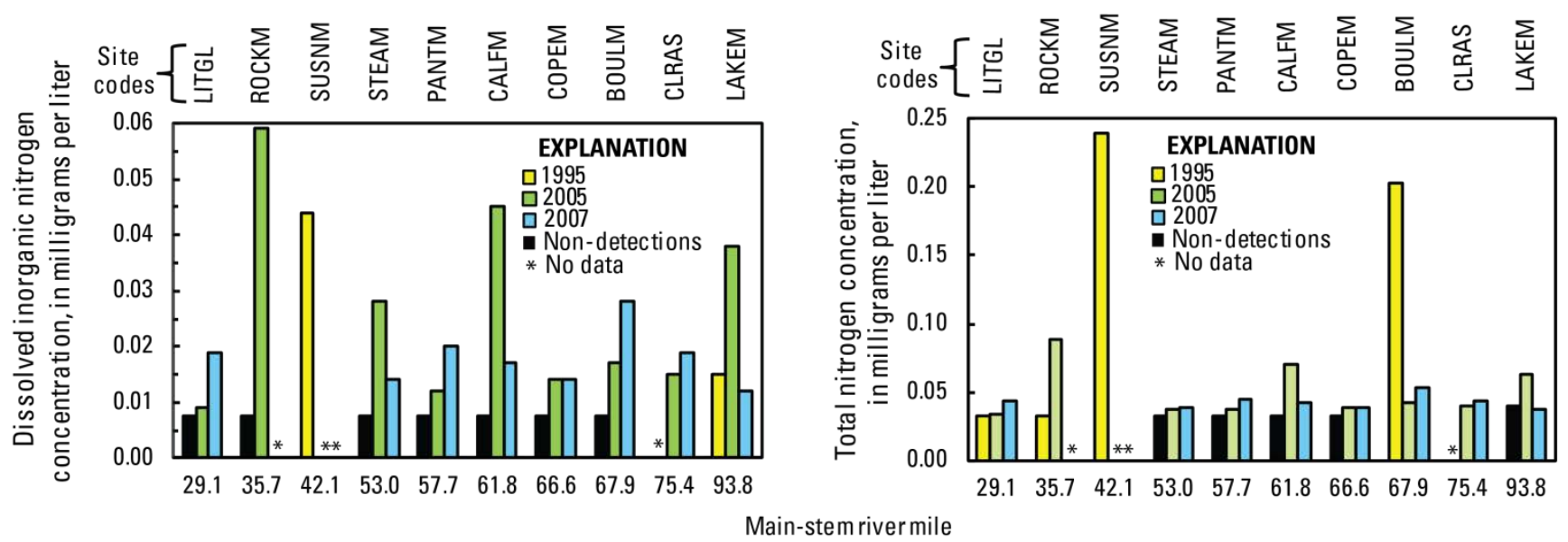

Figure 15. Longitudinal patterns in concentrations of dissolved inorganic nitrogen and total nitrogen in the $(A)$ mainstem North Umpqua River and (B) tributaries during the 1995, 2005, and 2007 synoptic surveys. Site codes are defined in table 5. 
Nutrient loads - the product of streamflow and nutrient concentration - are a useful metric for evaluating sources and sinks in river systems. This study did not exhaustively measure many of the terms for a detailed nutrient budget, such as nitrification and denitrification, reservoir internal loading, or uptake by algae, but instantaneous loads in the water column can identify potential source areas or sinks.

Dissolved and (or) total nitrogen loads were higher in 2005, prior to the rotenone application, than in 1995 in the bypass reach downstream of Lemolo Lake (at LEM1T/LEM1G), at NURCR, and in some tributaries such as Steamboat Creek (fig. 16). In contrast, lower streamflow at LAKEM led to lower DIN loads in 2005, and even lower in July 2007, when $\mathrm{NH}_{4}$ was below detection and $\mathrm{NO}_{3}$ was very low at $0.007 \mathrm{mg} / \mathrm{L}$. Low nitrogen loading at LAKEM during summer 2007 may be related to refilling of the lake with nitrogen-poor High Cascades water, and an absence of large blue-green algae blooms in Diamond Lake during summer 2007 (the year following the rotenone treatment).
The higher DIN load in 2005 (compared with 1995) at LEM1G was due to higher $\mathrm{NH}_{4}$ and $\mathrm{NO}_{3}$ concentrations, rather than higher discharges (figs. 15 and 16). These results suggest that Lemolo Lake grew as a nitrogen source from 1995 to 2005, probably from $\mathrm{N}$-fixing blue-green algae blooms there and in Diamond Lake, and perhaps from other upstream sources. The upper North Umpqua River and all eight tributaries sampled had higher $\mathrm{NO}_{3}$ in 2005 compared with 1995 (fig. 15), although the $\mathrm{NO}_{3}$ detections in the main stem in July 2005 were only slightly above the laboratory reporting level.

Lower streamflow in the upper portion of the Wild and Scenic Reach from 1995 to 2005 translated to lower DIN loads. But downstream at NURCR, DIN loads were higher in 2005, due to a small increase in $\mathrm{NO}_{3}$ of $0.0055 \mathrm{mg} / \mathrm{L}$, possibly from inputs of tributaries, groundwater, or other $\mathrm{NO}_{3}$ sources. 

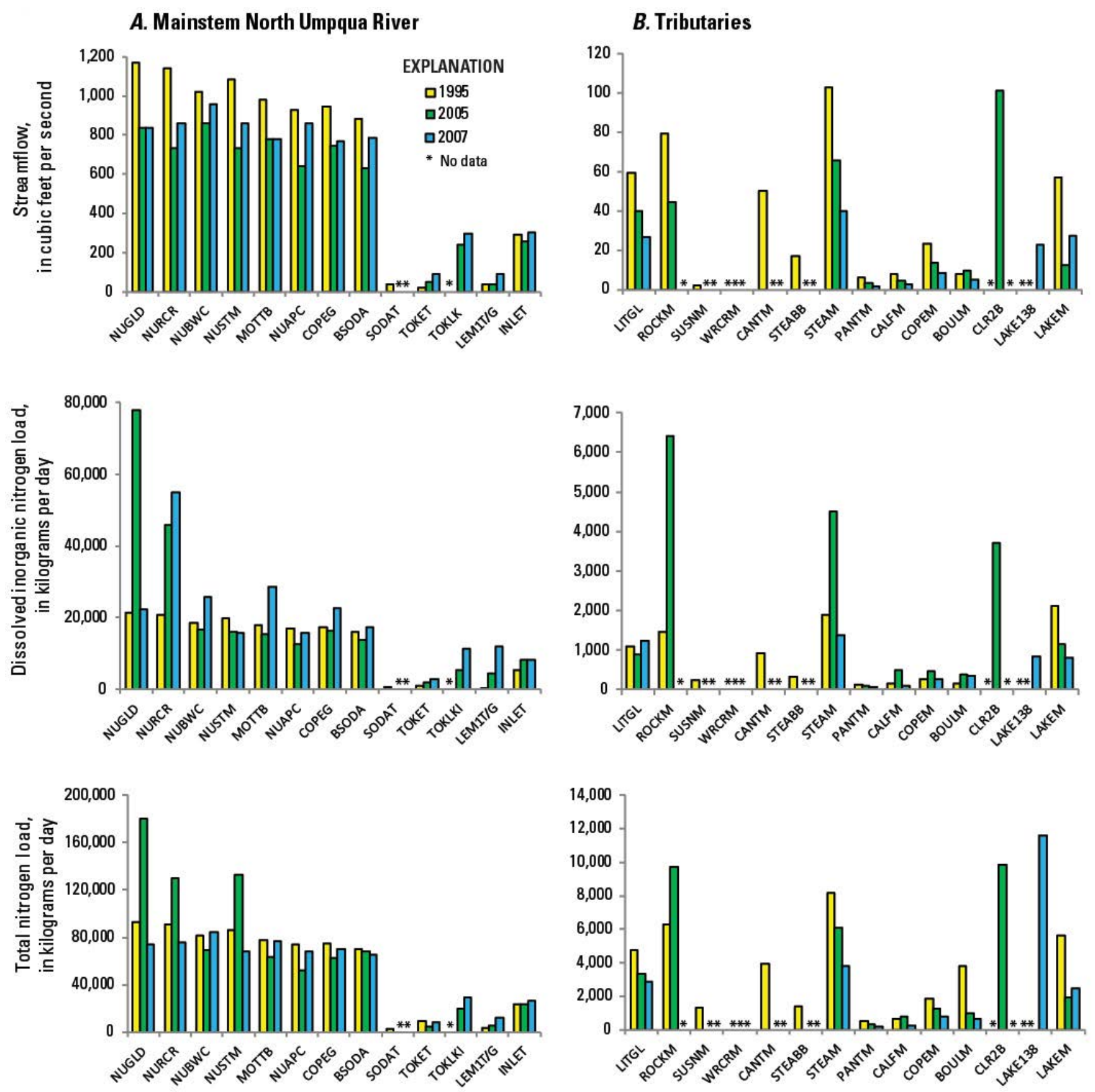

Sampling site

Figure 16. Streamflow and loads of nitrogen and phosphorus in the $(A)$ main-stem North Umpqua River and $(B)$ tributaries during the 1995, 2005, and 2007 synoptic surveys. Site codes are defined in table 5 . Figure continues on next page. 

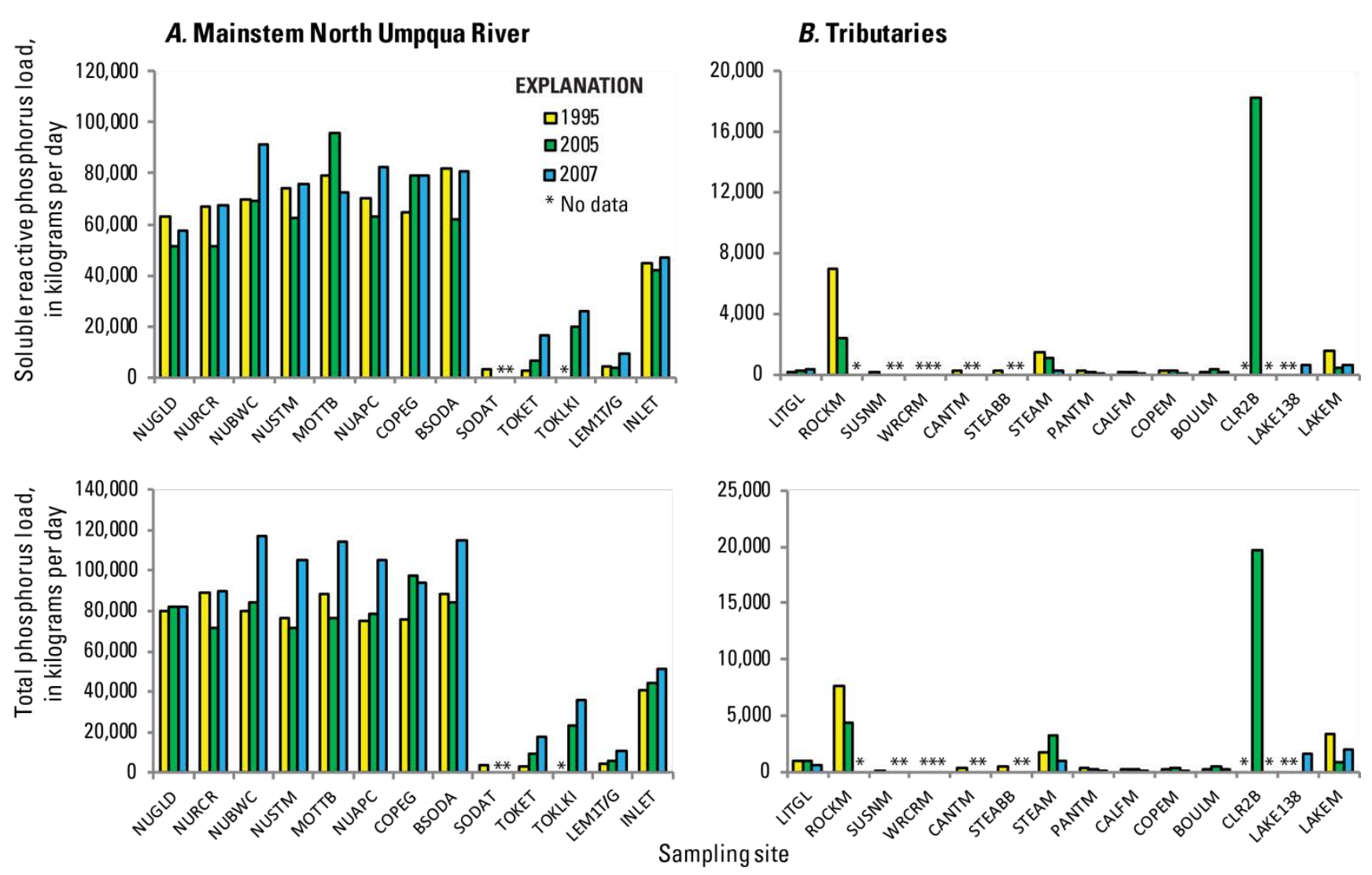

Figure 16 - continued. Streamflow and loads of nitrogen and phosphorus in the $(A)$ main-stem North Umpqua River and $(B)$ tributaries during the 1995, 2005, and 2007 synoptic surveys. Site codes are defined in table 5.

\section{Benthic Algal Conditions}

\section{Periphyton Biomass}

Chl- $a$ and AFDM, indicators of periphyton biomass, were used to examine temporal and longitudinal differences between 1995 and 2005, and 2005 and 2007 (fig. 17). Algal biomass can be an indicator of eutrophication in streams and rivers, but year-to-year variations in growing conditions and once-per-summer samplings preclude a precise comparison. Longitudinal patterns in biomass, however, can help identify downstream trends and areas that are prone to higher-level biomass.
Algal blooms (phytoplankton and periphyton) often are observed within the hydroelectric project, primarily in the reservoirs, canals, dam tailraces, and bypass reaches where habitat conditions are most suitable. The initial 1995 synoptic survey identified three sites within the project (LEM1G, TOKET, and BSODA) where periphyton biomass levels exceeded the 100-150 mg Chl- $a / \mathrm{m}^{2}$ nuisance threshold proposed by Welch and others $(1988,1989)$ (fig. 17). In 2007, periphyton biomass was much lower at LEM1G and TOKET, possibly because of higher in-stream flows required in the revised FERC operating license (table 3 ). 


\section{A. Algal biomass indicators}
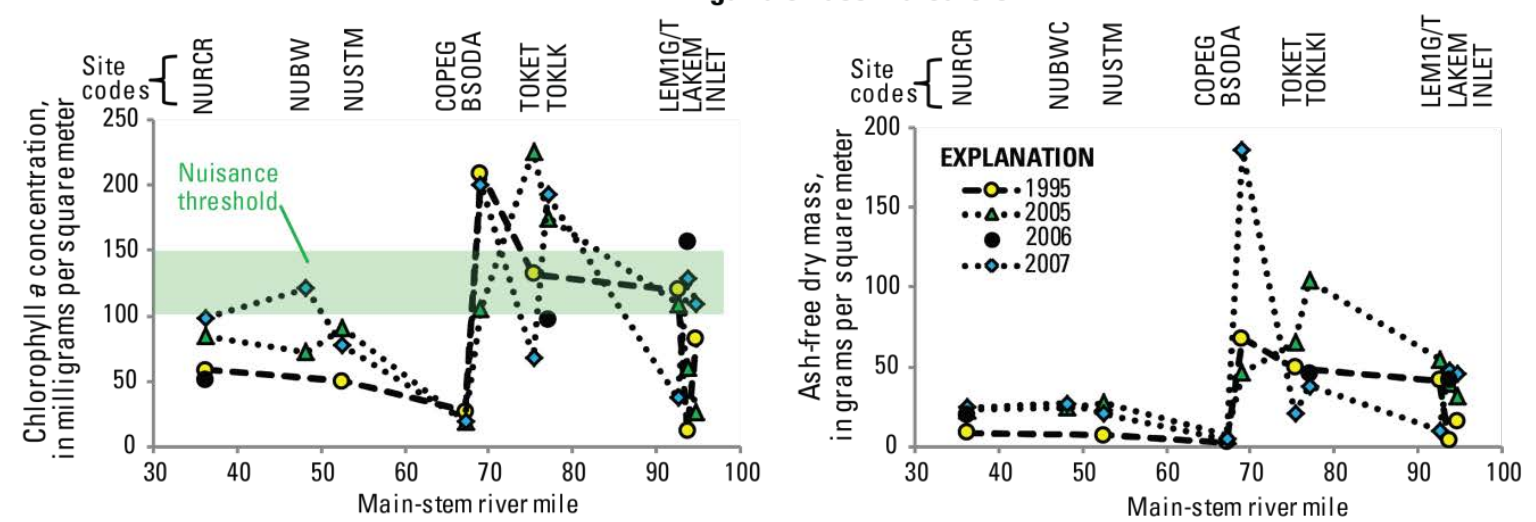

B. Algal-indicator guilds, in percent biovolume
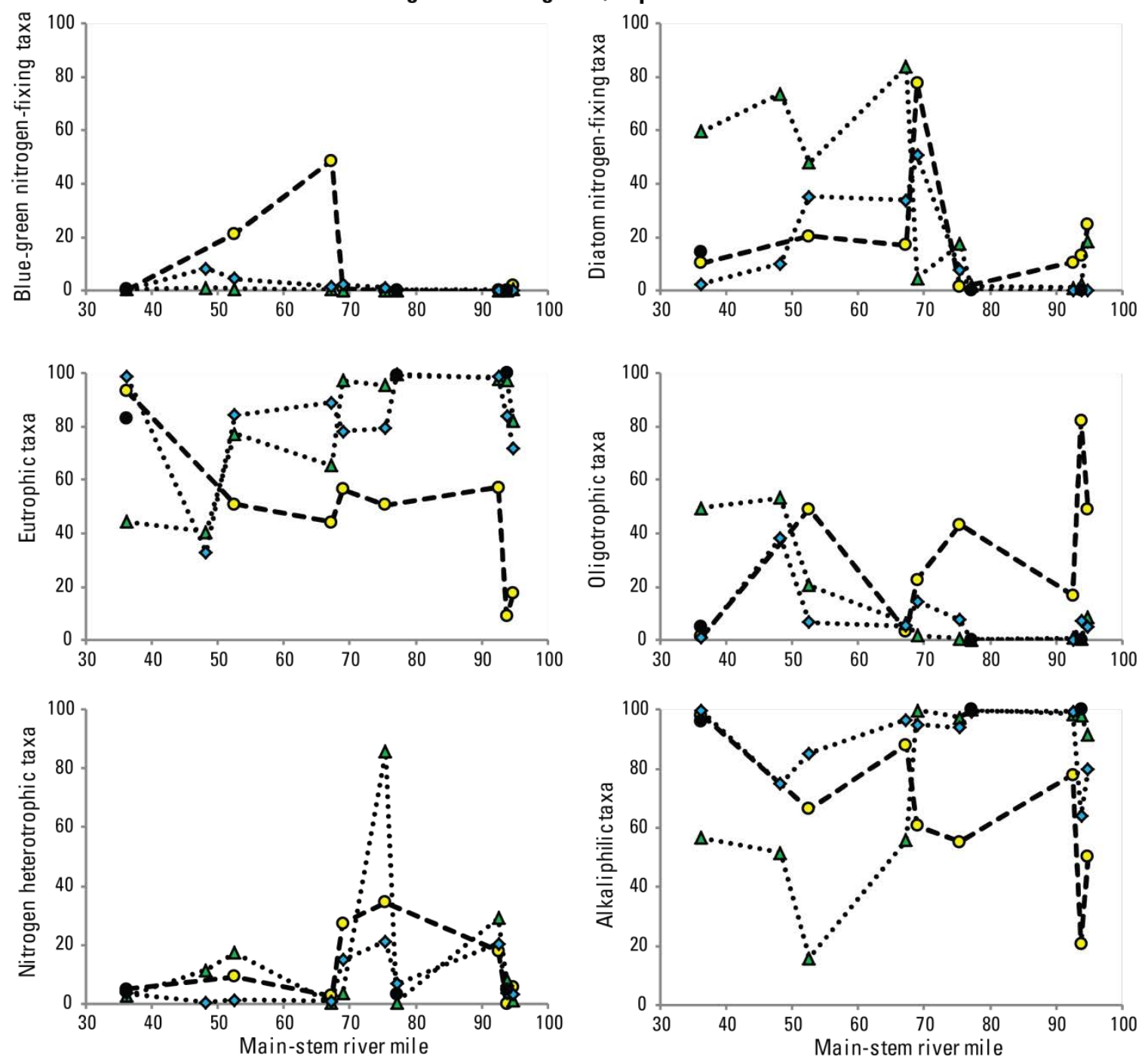

Figure 17. Patterns in $(A)$ periphyton biomass indicators and $(B)$ percent biovolume of selected algalindicator guilds in Lake Creek and the North Umpqua River during the 1995, 2005, and 2007 synoptic surveys. Nuisance chlorophyll-a threshold range of $100-150 \mathrm{mg} / \mathrm{m}^{2}$ suggested by Welch and others $(1988,1989)$. Site codes are defined in table 5. 
In the Wild and Scenic Reach, periphyton biomass was consistently low at COPEG (20-30 $\mathrm{mg}$ Chl $-a / \mathrm{m}^{2}$ ) but increased by two to four times downstream in all years, especially at sites downstream of Steamboat Creek (RM 53) (figs. 1 and 17). In 2007, the year after the rotenone treatment, Chl- $a$ met or exceeded the nuisance biomass level at two sites within the Wild and Scenic Reach (NUBWC and NURCR), consistent with greater concentrations of inorganic nitrogen from Diamond Lake (fig. 10). The lower biomass in the hydroelectric project bypass reaches in 2007 described previously also might have translated to increased growth downstream, as available nutrients were potentially transported to the lower Wild and Scenic Reach rather than incorporated into algal biomass within the project. Average Chl- $a$ values for the seven to eight main-stem sites sampled in 1995, 2005, and 2007 were within 6 percent of each other, ranging from $95-104 \mathrm{mg} / \mathrm{m}^{2}$, indicating relatively consistent, year-to-year growth patterns in the main stem.

\section{Periphyton Species Composition}

The harmonized dataset included 243 algal taxa from 5 major algal divisions for 1994-95 and 2005-07. Diatoms constituted 85 percent of the taxa, followed by green and blue-green algae (5-8 percent each), with less than 1 percent red and golden algal taxa. Diatoms represented most of the species richness in individual samples, whereas non-diatoms (soft algae) often dominated the total biovolume and cell density. The percent biovolume of soft algae, particularly green algae, was higher in 2005-07 than in 1995 at upperbasin sites. In contrast, diatoms were relatively more abundant in the downstream Wild and Scenic Reach sites in 2005-07 than in 1995 (table 11).

Algal species density and biovolume data are presented in appendix tables D2 and D3. Pooling data from all samples, small colonial forms of blue-green algae including Nostoc parmelioides and several types of filamentous blue-green algae (Leptolyngbya, Pseudanabaena, Phormidium, and Lyngbya) dominated the cell density, whereas large high-biomass forming taxa such as filamentous green algae (Cladophora glomerata, Oedogonium, Spirogyra, and Zygnema) constituted most of the overall algal biovolume. Among the diatoms, smaller taxa, such as Nitzschia tropica, Achnanthidium minutissimum, and Fragilaria vaucheriae, constituted most of the overall density, whereas relatively larger diatoms, including Epithemia spp., Nitzschia sp. 1, Synedra ulna, and Melosira varians, dominated the overall biovolume. Other taxa had high biovolumes in selected samples: the planktonic diatom Stephanodiscus niagarae at LAKEM in 2006; Cladophora glomerata at LAKEM in 2005-07, LEM1G in 2005 and 2007, TOKLKI in 2005-07, and NURCR in 2006-07; and Gomphoneis herculeana, a large stalked diatom at LAKEM in 2007 (Appendix D3, table 12, also see photograph 10). Gomphoneis herculeana also appeared at LEM1G in 1995 and 2005, STEAM in 2005 and 2007, and NURCR in July and September 1994 and 2006, but at lower abundance than the large bloom observed at LAKEM in 2007. 
Table 11. Distribution of major algal taxonomic divisions in samples from the North Umpqua River Basin, Oregon, 1995 and 2005-07.

[Data shown are percentages, based on percent biovolume. Percentages are rounded and might not add to 100 percent.]

\begin{tabular}{|c|c|c|c|c|c|c|c|}
\hline \multirow[b]{2}{*}{ Site } & \multirow[b]{2}{*}{ Year } & \multirow[b]{2}{*}{ Diatoms } & \multicolumn{5}{|c|}{ Soft Algae } \\
\hline & & & $\begin{array}{l}\text { Blue-green } \\
\text { algae }\end{array}$ & $\begin{array}{l}\text { Green } \\
\text { algae }\end{array}$ & $\begin{array}{r}\text { Red } \\
\text { algae }\end{array}$ & $\begin{array}{c}\text { Yellow- } \\
\text { green algae }\end{array}$ & $\begin{array}{l}\text { Total soft } \\
\text { algae }\end{array}$ \\
\hline LAKEM & 1995 & 72 & 28 & 0.0 & 0.0 & 0.6 & 28 \\
\hline LAKEM & 2005 & 8.7 & 0.3 & 91 & 0.0 & 0.0 & 91 \\
\hline LAKEM & 2006 & 0.2 & 0.0 & 100 & 0.0 & 0.0 & 100 \\
\hline LAKEM & 2007 & 39 & 2.2 & 59 & 0.0 & 0.0 & 61 \\
\hline$\overline{\text { INLET }}$ & 1995 & 96 & 3.9 & 0.3 & 0.0 & 0.2 & 4.4 \\
\hline INLET & 2005 & 55 & 4.9 & 40 & 0.0 & 0.0 & 45 \\
\hline INLET & 2007 & 60 & 12 & 27 & 0.0 & 0.0 & 40 \\
\hline$\overline{\text { LEM1T }}$ & 1995 & 100 & 0.0 & 0.4 & 0.0 & 0.0 & 0.4 \\
\hline LEM1G & 2005 & 7.0 & 0.1 & 93 & 0.0 & 0.0 & 93 \\
\hline LEM1G & 2007 & 3.1 & 2.1 & 95 & 0.0 & 0.0 & 97 \\
\hline TOKLKI & 2005 & 2.4 & 0.1 & 97 & 0.0 & 0.0 & 98 \\
\hline TOKLKI & 2006 & 1.5 & 0.0 & 98 & 0.0 & 0.0 & 98 \\
\hline TOKLKI & 2007 & 3.7 & 1.3 & 95 & 0.0 & 0.0 & 96 \\
\hline TOKET & 1995 & 99 & 0.0 & 0.8 & 0.0 & 0.0 & 0.8 \\
\hline TOKET & 2005 & 53 & 0.3 & 46 & 0.2 & 0.2 & 47 \\
\hline TOKET & 2007 & 52 & 10 & 38 & 0.0 & 0.2 & 48 \\
\hline$\overline{\text { BSODA }}$ & 1995 & 95 & 5.2 & 0.0 & 0.0 & 0.0 & 5.2 \\
\hline BSODA & 2005 & 9.2 & 0.2 & 91 & 0.0 & 0.0 & 91 \\
\hline BSODA & 2007 & 65 & 10 & 25 & 0.0 & 0.0 & 35 \\
\hline$\overline{\mathrm{COPEG}}$ & 1995 & 33 & 58 & 0.0 & 9.1 & 0.0 & 67 \\
\hline COPEG & 2005 & 97 & 1.1 & 0.6 & 0.9 & 0.0 & 2.5 \\
\hline COPEG & 2007 & 59 & 2.0 & 38 & 0.3 & 0.0 & 41 \\
\hline$\overline{\text { STEABB }}$ & 1995 & 99 & 1.1 & 0.3 & 0.0 & 0.0 & 1.3 \\
\hline STEAM & 1995 & 38 & 38 & 24 & 0.0 & 0.0 & 62 \\
\hline STEAM & 2005 & 96 & 1.4 & 3.0 & 0.0 & 0.0 & 4.4 \\
\hline STEAM & 2007 & 90 & 4.4 & 5.5 & 0.0 & 0.0 & 10 \\
\hline$\overline{\text { NUSTM }}$ & 1995 & 38 & 62 & 0.0 & 0.0 & 0.1 & 62 \\
\hline NUSTM & 2005 & 96 & 3.6 & 0.4 & 0.0 & 0.0 & 4.0 \\
\hline NUSTM & 2007 & 69 & 8.6 & 22 & 0.0 & 0.0 & 31 \\
\hline$\overline{\text { NUBWC }}$ & 2005 & 97 & 1.7 & 1.6 & 0.0 & 0.0 & 3.3 \\
\hline NUBWC & 2007 & 75 & 21 & 4.0 & 0.0 & 0.0 & 25 \\
\hline NURCR1 & 1994 & 92 & 8.3 & 0.0 & 0.0 & 0.0 & 8.3 \\
\hline NURCR1 & 1994 & 93 & 4.9 & 1.9 & 0.0 & 0.0 & 6.7 \\
\hline NURCR1 & 1995 & 24 & 4.2 & 72 & 0.0 & 0.0 & 76 \\
\hline NURCR2 & 1995 & 67 & 24 & 9.0 & 0.0 & 0.0 & 33 \\
\hline NURCR1 & 2005 & 98 & 1.5 & 0.6 & 0.0 & 0.0 & 2.1 \\
\hline NURCR2 & 2005 & 61 & 13 & 25 & 0.0 & 0.0 & 39 \\
\hline NURCR1 & 2006 & 41 & 10 & 49 & 0.0 & 0.0 & 59 \\
\hline NURCR1 & 2007 & 9.1 & 3.6 & 87 & 0.0 & 0.0 & 91 \\
\hline NURCR2 & 2007 & 35 & 34 & 32 & 0.0 & 0.0 & 65 \\
\hline LITGL & 2007 & 8.8 & 25 & 67 & 0.0 & 0.0 & 91 \\
\hline
\end{tabular}


Table 12. Top five most abundant algal taxa based on percent biovolume for selected sites in the North Umpqua River basin, Oregon, 1995 and 2005-07.

[Colors indicate green algae, blue-green algae, and diatoms. --, no data]

\begin{tabular}{|c|c|c|c|}
\hline \multicolumn{4}{|c|}{ Year } \\
\hline 1995 & 2005 & 2006 & 2007 \\
\hline \multicolumn{4}{|c|}{ Lake Creek (LAKEM) } \\
\hline Gomphonema subclavatum & Cladophora glomerata & Cladophora glomerata & Cladophora glomerata \\
\hline Epithemia spp. & Epithemia spp. & Stephanodiscus niagarae & Eudorina elegans \\
\hline Cocconeis sp. & Gomphoneis mammilla & Nitzschia sp. 1 & Gomphoneis herculeana \\
\hline Hannaea arcus & Encyonema silesiacum & Filamentous blue-green algae ${ }^{1}$ & Cymbella subturgidula \\
\hline Synedra ulna & Cocconeis sp. & Epithemia spp. & Synedra ulna \\
\hline \multicolumn{4}{|c|}{ North Umpqua River near Inlet Campground (INLET) } \\
\hline Epithemia spp. & Zygnema sp. & -- & Zygnema sp. \\
\hline Diatoma vulgaris & Epithemia spp. & -- & Filamentous blue-green algae $\mathrm{e}^{1}$ \\
\hline Cymbella mexicana & Gomphoneis mammilla & -- & Gomphoneis herculeana \\
\hline Hannaea arcus & Cocconeis sp. & -- & Synedra ulna \\
\hline Diatoma mesodon & $\begin{array}{l}\text { Unknown coccoid blue-green } \\
\text { alga }\end{array}$ & -- & Gomphoneis minuta \\
\hline \multicolumn{4}{|c|}{ North Umpqua River above Copeland Creek (COPEG) } \\
\hline Nostoc parmeloides & Epithemia spp. & -- & Epithemia spp. \\
\hline Epithemia spp. & Cocconeis sp. & -- & Zygnema sp. \\
\hline Rivulariaceae & Gomphonema rhombicum & -- & Cocconeis sp. \\
\hline Unknown red alga & Encyonema silesiacum & -- & Gongrosira sp. \\
\hline Cocconeis sp. & Unknown red alga & -- & Gomphoneis herculeana \\
\hline \multicolumn{4}{|c|}{ Steamboat Creek (STEAM) } \\
\hline Rivulariaceae & Epithemia sp. & -- & Cymbella subturgidula \\
\hline Zygnema sp. & Cymbella subturgidula & -- & Epithemia spp. \\
\hline Epithemia sp. & Cymbella mexicana & -- & Gomphoneis herculeana \\
\hline Synedra ulna & Gomphoneis herculeana & -- & Synedra ulna \\
\hline Filamentous blue-green algae $^{1}$ & Staurastrum punctulatum & -- & Rhopalodia gibba \\
\hline \multicolumn{4}{|c|}{ North Umpqua River above Rock Creek (NURCR) } \\
\hline Zygnema sp. & Epithemia spp. & Cladophora glomerata & Cladophora glomerata \\
\hline Epithemia spp. & Spirogyra sp. & Zygnema sp. & Filamentous blue-green algae $\mathrm{e}^{1}$ \\
\hline Rivulariaceae & Filamentous blue-green algae ${ }^{1}$ & Epithemia spp. & Spirogyra sp. \\
\hline Synedra ulna & Cymbella mesiana & Filamentous blue-green algae ${ }^{1}$ & Zygnema sp. \\
\hline Stigeoclonium lubricum & Cymbella mexicana & Nitzschia sp. 1 & Nitzschia sp. 1 \\
\hline
\end{tabular}

\footnotetext{
${ }^{1}$ Filamentous blue-green algae includes Phormidium autumnale, Phormidium sp., Leptolyngbya sp., Lyngbya martensiana,
} Homoeothrix janthina, $H$. juliana, $H$. varians, and Oscillatoria sp. 


\section{Periphyton Guilds}

About 70 percent of the 243 taxa were classified into one or more guilds (table 13). Some guilds had significant Spearman rank correlations $(\mathrm{P}<0.05$ or less $)$ with their corresponding variables, but correlations between guilds and associated water-quality variables were not always significant. The $\mathrm{N}$-fixing guilds - diatom and blue-green $\mathrm{N}$-fixers - were negatively correlated with concentrations of $\mathrm{NO}_{3}$ and $\mathrm{NH}_{4}$ (appendix table D4), but eutrophic taxa were not correlated with nutrient concentrations. $\mathrm{NO}_{3}$ concentrations were positively correlated with total algal biovolume $(\mathrm{P}<0.01)$ and total biovolume of green algae $(\mathrm{P}<0.05)$, whereas TP and SRP were significantly correlated with percent biovolume of mesotrophic taxa, Nheterotrophic taxa, and minimum DO concentrations.

Table 13. Description of periphyton indicator-species guilds identified from samples collected in the North Umpqua River basin, Oregon, 1995 and 2005-07.

[Guild definitions from Porter, 2008]

\begin{tabular}{|c|c|c|c|}
\hline Periphyton indicator guild & Abbreviation & Algal taxon & Definition \\
\hline Alkalibiontic taxa & Alk-B & All taxa & $\mathrm{pH}$ optima greater than $7-8$ standard $\mathrm{pH}$ units \\
\hline Alkaliphilic taxa & Alk-P & All taxa & $\mathrm{pH}$ optima greater than 7 standard $\mathrm{pH}$ units \\
\hline All eutrophic taxa & $\mathrm{E}$ & All taxa & Optimal growth at high nutrient concentrations \\
\hline Eutrophic taxa & $\mathrm{E}$ & Diatoms & Optimal growth at high nutrient concentrations \\
\hline Mesotrophic taxa & M & Diatoms & Optimal growth at moderate nutrient concentrations \\
\hline Nitrogen-heterotrophic taxa & $\mathrm{NH}$ & Diatoms & Ability to utilize organic nitrogen \\
\hline Nitrogen-fixing taxa & NF & All taxa & Ability to fix atmospheric nitrogen \\
\hline Oligotrophic taxa & $\mathrm{O}$ & Diatoms & Tolerates or prefers low nutrient concentrations \\
\hline $\begin{array}{l}\text { Organic pollution-sensitive } \\
\text { diatoms }\end{array}$ & OP-S & Diatoms & $\begin{array}{l}\text { Somewhat intolerant to nutrient and organic enrichment } \\
\text { not necessarily "oligotrophic"; class: I, I-II; } \mathrm{O}_{2} \\
\text { saturation: greater than } 85 \% \text {; } \mathrm{BOD}_{5}(\mathrm{mg} / \mathrm{L}) \text { : less than } 2 \text {; } \\
\text { oligosaprobic: low amounts of organic enrichment; } \\
\text { mesotrophic; oligo-mesotrophic (but not necessarily } \\
\text { pristine) }\end{array}$ \\
\hline Oxygen-high & $\mathrm{OXY}-\mathrm{H}$ & Diatoms & $\begin{array}{l}\text { Oxygen concentrations always high, nearly } 100 \% \\
\text { saturation }\end{array}$ \\
\hline
\end{tabular}

Longitudinal and annual patterns in percent biovolume of selected algal guilds in the mainstem North Umpqua River are shown in figure 17 , with specific data for percent biovolume of each guild listed in table 14. Percent biovolume of $\mathrm{N}$-fixers generally increased downstream in the main stem, with diatoms, primarily Epithemia spp., comprising the majority (fig. 17). Epithemia spp. includes large diatoms that contain endosymbiotic cells of $\mathrm{N}$-fixing blue-green algae. This genus is common in the nitrogen-limited streams of the Cascade Range (Carpenter, 2003; Bahls, 2004; Bahls, 2005).

In 2005, the percent biovolume of blue-green $\mathrm{N}$-fixers was lower than in 1995, and diatom $\mathrm{N}$ fixers were higher, particularly in the Wild and Scenic Reach, which partly explains the observed difference in diatoms. Blue-green $\mathrm{N}$-fixers, primarily Nostoc parmelioides and Calothrix sp., were more abundant than diatom $\mathrm{N}$-fixers at COPEG in 1995, but in 2005 and 2007, diatom $\mathrm{N}$-fixers were more abundant based on both the absolute and percent biovolume. 
Table 14. Periphyton indicator-species guild values for samples collected from the North Umpqua River basin, Oregon, 1995 and 2005-07.

[Guild values based on percent biovolume. Guild definitions are given in table 13. Site codes shown in table 5. Guild percentages based on "classified" taxa only (see Methods). Values greater than 25 percent are bolded. Abbreviations: a, indicates algal samples identified by the Philadelphia Academy of Natural Sciences; j, indicates algal samples identified by Jim Sweet, Aquatic Analysts].

\begin{tabular}{|c|c|c|c|c|c|c|c|c|c|c|c|c|}
\hline \multirow[b]{2}{*}{ Site Code } & \multirow[b]{2}{*}{ Year } & \multirow[b]{2}{*}{ Sample Code } & \multicolumn{10}{|c|}{ Periphyton Indicator Guild } \\
\hline & & & 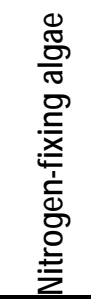 & 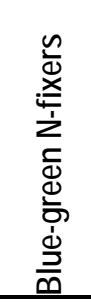 & 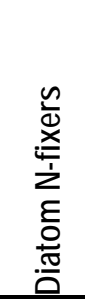 & 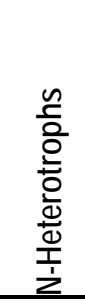 & 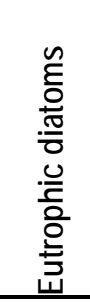 & 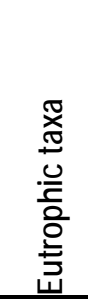 & 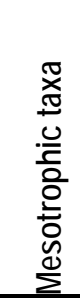 & 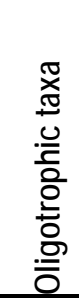 & 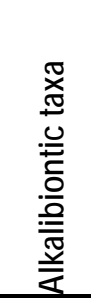 & 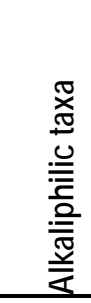 \\
\hline LAKEM & 1995 & LAKEM_95j & 13.0 & 0.0 & 13.0 & 0.8 & 9.0 & 9.0 & 0.1 & 82.3 & 0.0 & 20.6 \\
\hline LAKEM & 2005 & LAKEM_05 & 2.4 & 0.0 & 2.3 & 8.1 & 18.8 & 97.2 & 0.2 & 0.6 & 0.0 & 97.9 \\
\hline LAKEM & 2006 & LAKEM_06 & 0.0 & 0.0 & 0.0 & 4.9 & 63.5 & 99.9 & 0.0 & 0.0 & 0.0 & 100.0 \\
\hline LAKEM & 2007 & LAKEM_07 & 0.1 & 0.1 & 0.0 & 3.7 & 57.4 & 83.8 & 0.8 & 7.1 & 0.0 & 64.1 \\
\hline INLET & 1995 & INLET_95j & 26.6 & 1.9 & 24.7 & 6.6 & 17.3 & 17.7 & 46.7 & 48.9 & 21.9 & 50.4 \\
\hline INLET & 2005 & INLET_05 & 18.9 & 0.3 & 18.6 & 1.2 & 30.5 & 82.3 & 5.4 & 8.5 & 73.4 & 91.5 \\
\hline INLET & 2007 & INLET 07 & 0.2 & 0.2 & 0.0 & 3.5 & 55.3 & 71.6 & 5.9 & 4.9 & 46.4 & 79.8 \\
\hline LEM1T & 1995 & LEM1T_95j & 10.5 & 0.0 & 10.5 & 30.1 & 56.9 & 57.1 & 27.9 & 16.9 & 15.7 & 78.0 \\
\hline LEM1G & 2005 & LEM1G_05 & 1.1 & 0.0 & 1.1 & 29.5 & 62.8 & 98.0 & 0.3 & 0.8 & 2.2 & 98.6 \\
\hline LEM1G & 2007 & LEM1G_07 & 0.2 & 0.2 & 0.0 & 20.3 & 43.0 & 98.8 & 0.2 & 0.2 & 0.0 & 99.5 \\
\hline TOKLKI & 2005 & TOKLKI 05 & 1.7 & 0.1 & 1.6 & 1.0 & 37.6 & 99.8 & 0.0 & 0.1 & 0.4 & 99.9 \\
\hline TOKLKI & 2006 & TOKLKI_06 & 0.2 & 0.0 & 0.2 & 3.1 & 21.0 & 99.3 & 0.1 & 0.0 & 0.0 & 100.0 \\
\hline TOKLKI & 2007 & TOKLKI_07 & 0.5 & 0.1 & 0.4 & 6.7 & 40.3 & 99.2 & 0.0 & 0.3 & 0.1 & 99.5 \\
\hline TOKET & 1995 & TOKET_95j & 1.3 & 0.0 & 1.3 & 35.0 & 50.3 & 50.8 & 36.7 & 43.3 & 0.0 & 55.3 \\
\hline TOKET & 2005 & TOKET_05 & 17.6 & 0.2 & 17.4 & 85.9 & 89.4 & 95.6 & 0.5 & 0.5 & 0.0 & 97.5 \\
\hline TOKET & 2007 & TOKET_-07 & 8.5 & 1.0 & 7.5 & 21.4 & 43.0 & 79.5 & 2.2 & 7.6 & 3.0 & 93.9 \\
\hline BSODA & 1995 & BSODA_95j & 78.4 & 0.9 & 77.6 & 32.8 & 56.5 & 56.5 & 20.5 & 22.6 & 4.6 & 60.7 \\
\hline BSODA & 2005 & BSODA_05 & 4.8 & 0.1 & 4.7 & 3.5 & 15.7 & 97.4 & 0.0 & 1.8 & 98.8 & 99.7 \\
\hline BSODA & 2007 & BSODA_07 & 52.8 & 2.2 & 50.6 & 15.9 & 31.1 & 78.3 & 2.1 & 14.6 & 48.7 & 94.9 \\
\hline COPEG & 1995 & COPEG 95a & 65.5 & 48.6 & 16.9 & 4.4 & 44.0 & 44.0 & 9.9 & 3.2 & 6.7 & 88.0 \\
\hline COPEG & 2005 & COPEG_05 & 84.3 & 0.4 & 83.9 & 0.2 & 65.3 & 65.4 & 1.2 & 6.3 & 0.0 & 55.8 \\
\hline COPEG & 2007 & COPEG_07 & 35.3 & 1.5 & 33.8 & 1.1 & 56.9 & 89.0 & 1.0 & 5.4 & 86.3 & 96.5 \\
\hline STEABB & 1995 & STEABB $95 \mathrm{j}$ & 90.1 & 1.1 & 89.1 & 2.2 & 89.4 & 89.4 & 0.0 & 0.4 & 84.5 & 97.7 \\
\hline STEAM & 1995 & STEAM 95a & 45.6 & 30.2 & 15.4 & 4.0 & 53.3 & 77.2 & 0.0 & 4.3 & 62.7 & 98.3 \\
\hline STEAM & 2005 & STEAM_05 & 63.5 & 0.2 & 63.4 & 9.0 & 28.7 & 28.7 & 0.1 & 61.4 & 3.6 & 56.1 \\
\hline STEAM & 2007 & STEAM_07 & 19.7 & 2.0 & 17.6 & 3.9 & 26.1 & 31.0 & 0.0 & 54.9 & 20.4 & 74.5 \\
\hline NUSTM & 1995 & NUSTM_95j & 41.5 & 21.3 & 20.2 & 9.1 & 50.9 & 50.9 & 0.0 & 49.1 & 0.0 & 66.3 \\
\hline NUSTM & 2005 & NUSTM_05 & 48.7 & 0.7 & 48.0 & 17.4 & 77.3 & 77.3 & 0.8 & 20.6 & 3.0 & 15.9 \\
\hline NUSTM & 2007 & NUSTM_07 & 39.5 & 4.3 & 35.1 & 1.5 & 60.9 & 84.4 & 0.8 & 6.7 & 48.2 & 85.2 \\
\hline NUBWC & 2005 & NUBWC 05 & 74.9 & 1.1 & 73.8 & 11.3 & 37.9 & 40.6 & 1.8 & 53.4 & 0.2 & 51.6 \\
\hline NUBWC & 2007 & NUBWC_07 & 18.3 & 8.2 & 10.1 & 0.5 & 24.8 & 32.9 & 1.8 & 38.2 & 13.9 & 74.9 \\
\hline NURCR & 1994 & NURCR 94-7 & 21.3 & 2.1 & 19.2 & 11.2 & 25.3 & 25.3 & 21.0 & 16.4 & 25.0 & 75.8 \\
\hline NURCR & 1994 & NURCR 94-9 & 14.4 & 2.8 & 11.6 & 11.0 & 39.5 & 41.0 & 20.6 & 13.4 & 36.7 & 87.9 \\
\hline NURCR & 1995 & NURCR_95a & 10.6 & 0.5 & 10.2 & 10.0 & 48.3 & 93.3 & 1.9 & 1.3 & 93.3 & 98.2 \\
\hline NURCR & 2005 & NURCR_05 & 60.0 & 0.3 & 59.7 & 2.5 & 44.3 & 44.3 & 0.8 & 49.4 & 6.8 & 56.6 \\
\hline NURCR & 2006 & NURCR_06 & 14.4 & 0.0 & 14.4 & 4.2 & 28.2 & 82.9 & 0.4 & 4.8 & 33.0 & 96.2 \\
\hline NURCR & 2007 & NURCR_07 & 2.7 & 0.3 & 2.4 & 10.1 & 40.2 & 98.6 & 0.0 & 0.9 & 21.1 & 99.7 \\
\hline LITGL & 2007 & LITGL 07 & 55.3 & 18.3 & 37.0 & 6.6 & 29.2 & 47.1 & 4.1 & 15.7 & 6.2 & 92.1 \\
\hline
\end{tabular}


The percent biovolume of eutrophic taxa was higher at many sites in 2005 than in1995, whereas oligotrophic diatoms had generally lower percent biovolume (fig. 17). Nitrogen heterotrophic diatoms (taxa that can use dissolved organic nitrogen) had higher percent biovolume in 1995 compared with 2007, with the highest values consistently occurring at TOKET, downstream from Toketee Lake. Alkaliphilic taxa and eutrophic taxa, which indicate elevated nutrient conditions, dominated the algal guilds in the restoration-affected reach from LAKEM downstream to the North Umpqua River (figs. 17 and 18). In 2007, the average percent biovolumes of $\mathrm{N}$-fixing taxa was higher, and $\mathrm{N}$-heterotrophs lower, than in 2005. In 2005 and 2007, a larger abundance of filamentous green algae accounted for the changes in eutrophic taxa (figs. 18 and 19).

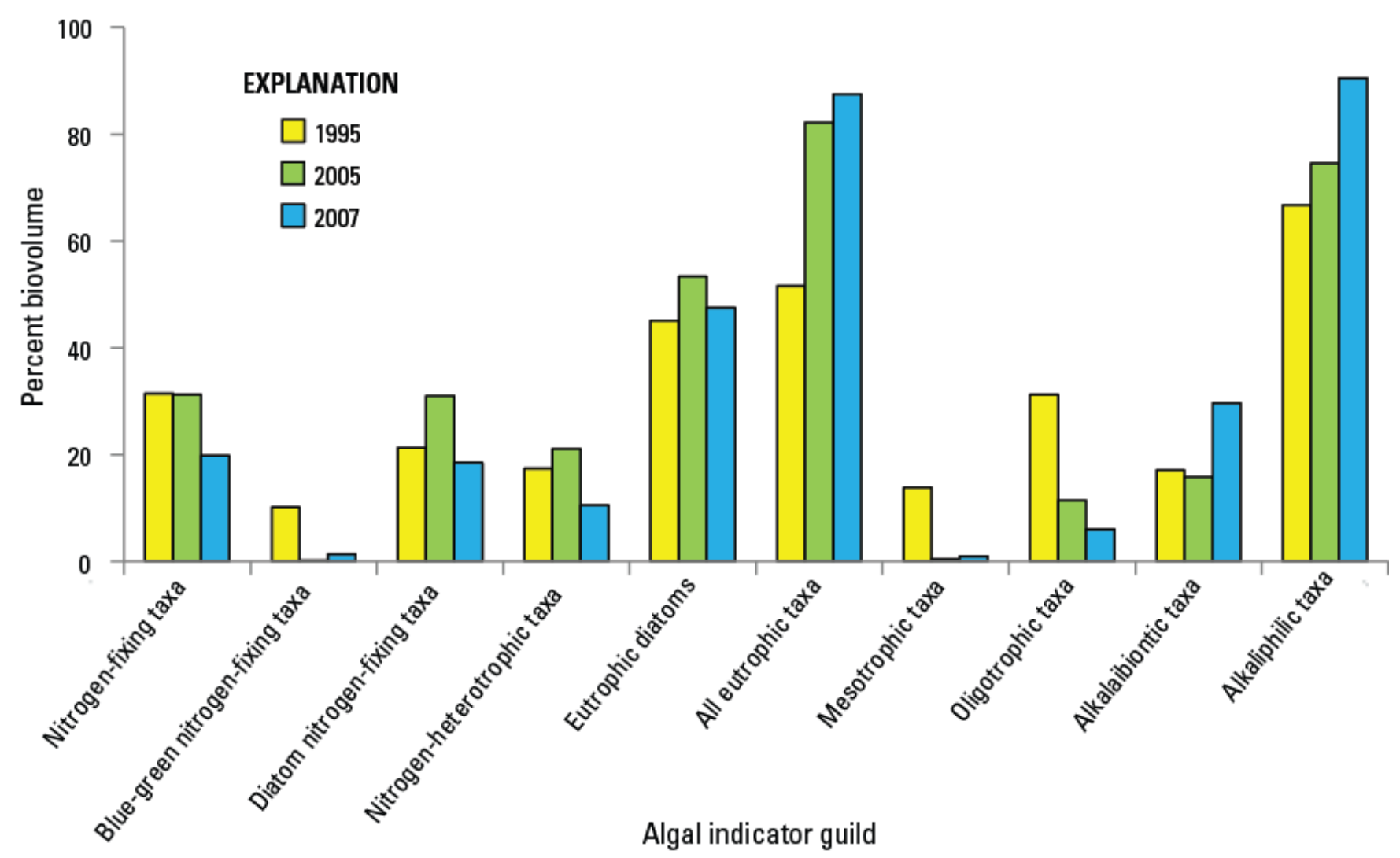

Figure 18. Percent biovolume of algal-indicator guilds in Lake Creek and the North Umpqua River during the 1995, 2005, and 2007 synoptic surveys. For emphasis on the Diamond Lake-affected reach and for equal comparisons among years, plot excludes the upper North Umpqua River upstream of Lemolo Lake (INLET), tributaries downstream of Lemolo Lake, and sites that were not sampled in 1995, including the North Umpqua River sites at the inlet to Toketee Lake (TOKLKI) and downstream of Wright Creek (NUWBC). 


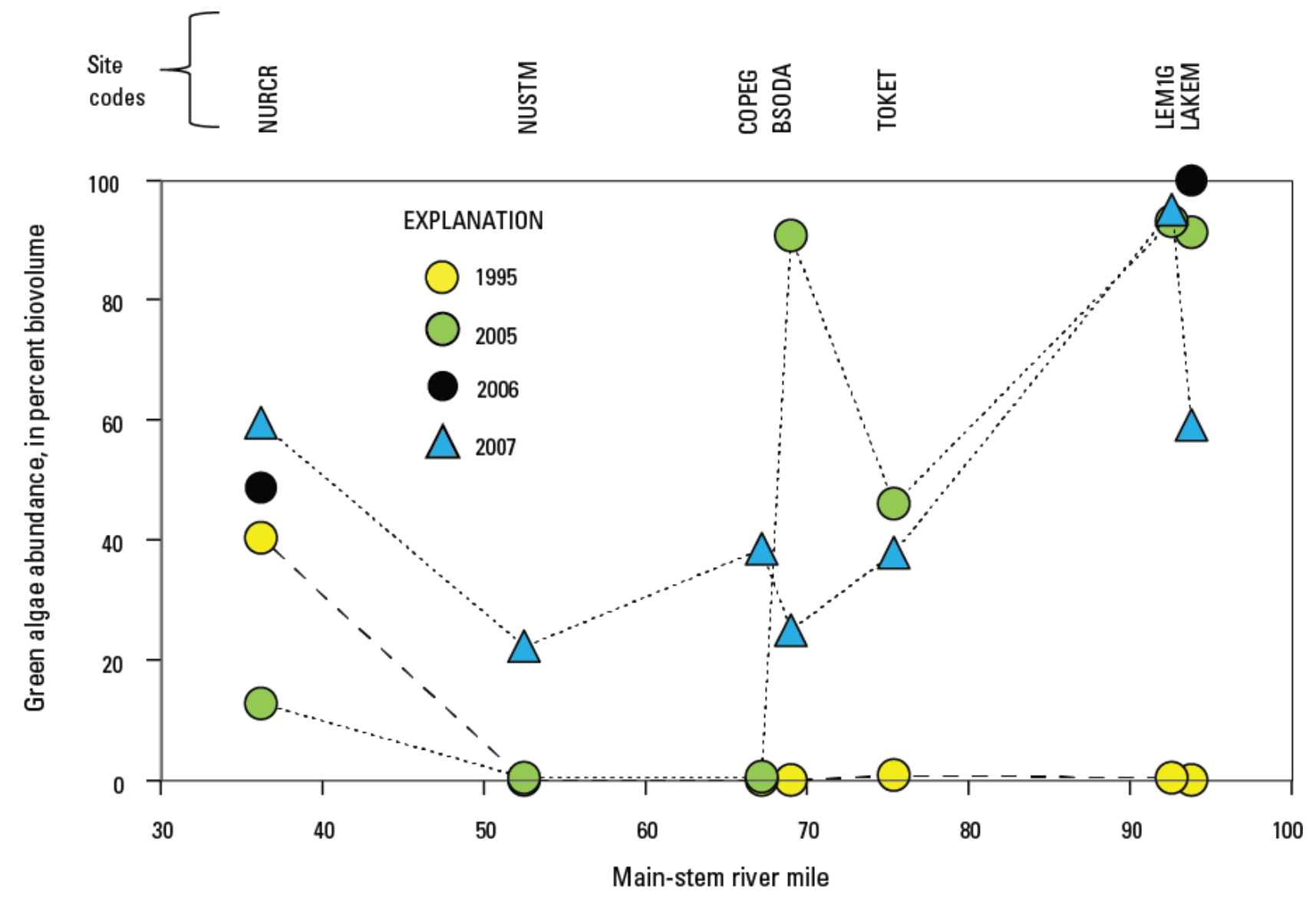

Figure 19. Percent biovolume of green algae in Lake Creek and the North Umpqua River during the 1995, 2005, and 2007 synoptic surveys. For emphasis on the Diamond Lake-affected reach and for equal comparisons among years, plot excludes the upper North Umpqua River upstream of Lemolo Lake (INLET), other tributaries downstream of Lemolo Lake, and sites that were not sampled in 1995, including the North Umpqua River sites at the inlet to Toketee Lake (TOKLKI) and downstream of Wright Creek (NUWBC).

\section{Changes in Algal Species Composition between 1995 and 2005}

Based on NMDS ordination of diatoms and soft algae, species compositions in 2005 were distinct from those in $1995(\mathrm{P}<0.001)$, with clear separation of samples between years (fig. 20). At least some of the separation is due to differences in algal taxonomy and particular methods used by the two laboratories as discussed in the section, "Methods." The number of cells enumerated by the ANSP for the 2005 samples was more than 3 times greater than those enumerated by Aquatic Analysts. This methodological difference probably contributed to the greater species richness in 2005 (44 \pm 9 taxa) than in $1995(21 \pm 7$ taxa) and resulted in 36 new diatom taxa that were absent in 1995. Additionally, Aquatic Analysts identified 11 taxa in 1995 that were not detected in the 2005 samples. Some of the differences in taxonomy between the contractors were addressed by grouping varieties and species together into a higher taxonomic level, as detailed in the Methods section. Although this approach diminishes the distortion from the laboratory method differences and makes for a better comparison between years, some species and subspecies information is obscured. 
A. Samples collected in 1995 and 2005

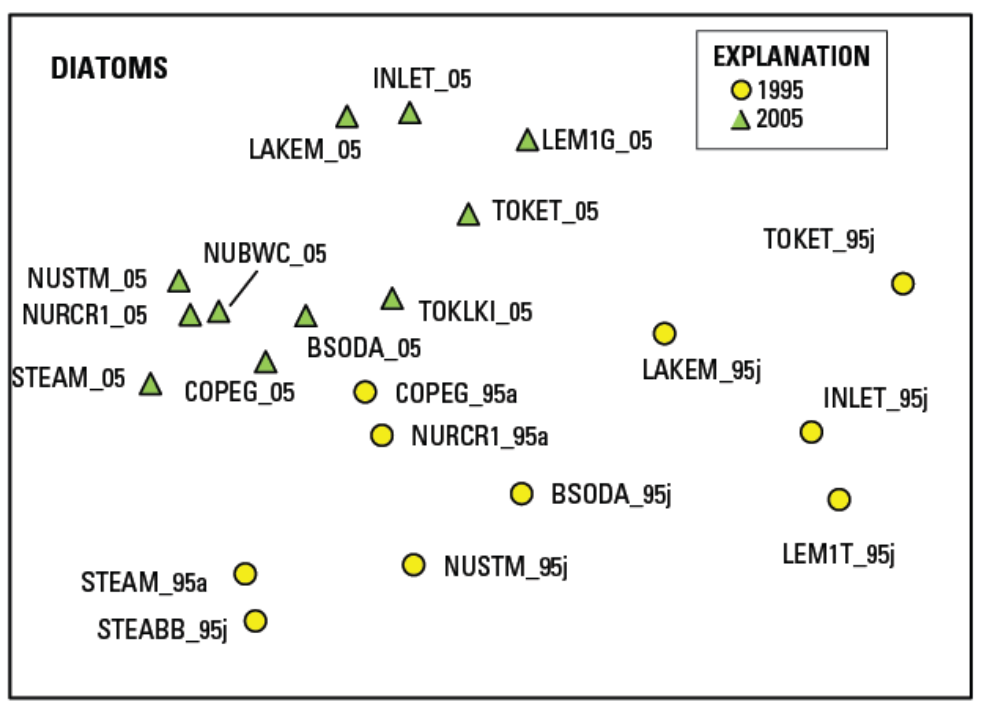

is
B. Samples collected in 2005, 2006, and 2007

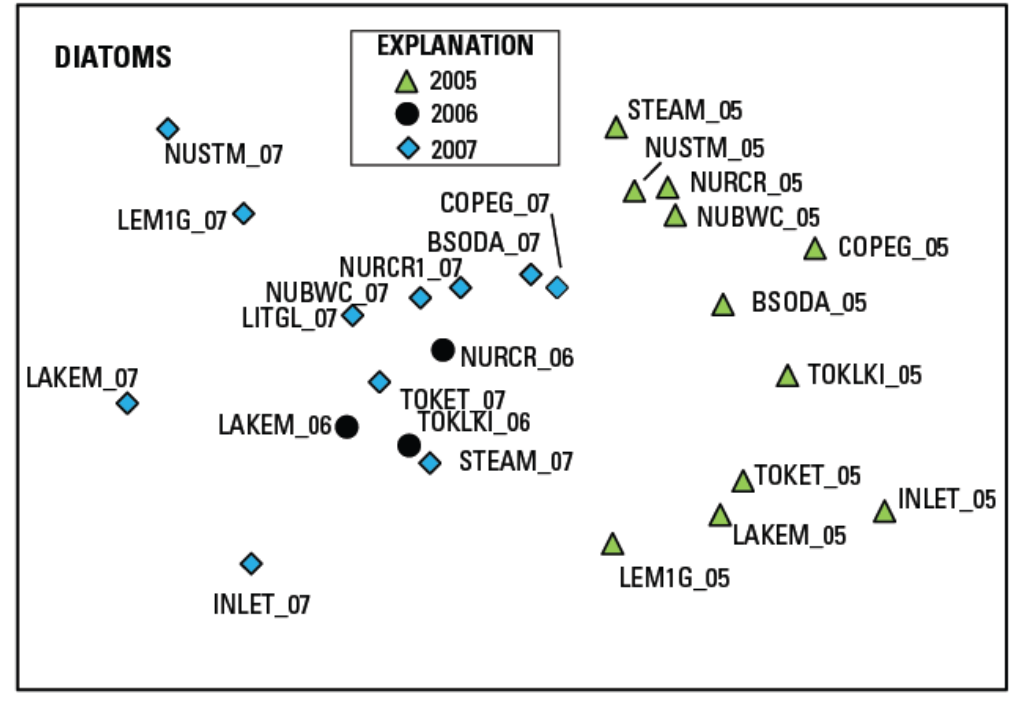

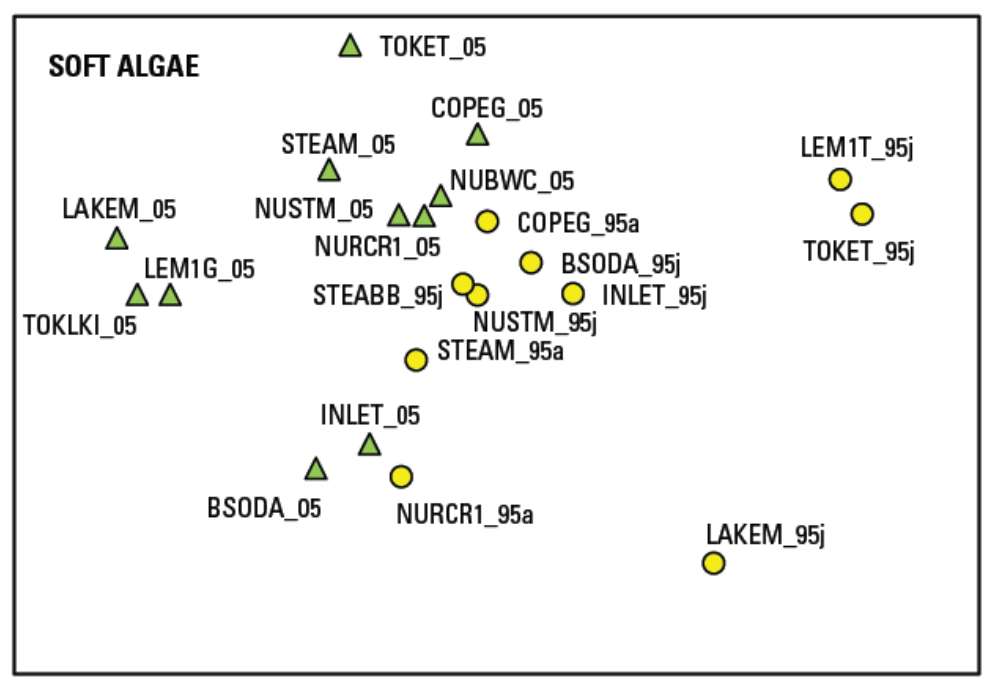

Figure 20. Ordination diagrams of algae samples from the North Umpqua River basin, Oregon, based on diatoms and soft algae for $(A)$ samples collected in 1995 and 2005, and (B) samples collected in 2005-07. Ordinations are based on percent biovolume of each alga taxon. Site codes are defined in table 5. 
Species compositions differed significantly between 1995 and 2005 for diatoms $(\mathrm{P}<0.001)$ and soft algae $(\mathrm{P}<0.01)$. The most notable difference was in the percent biovolume of diatom species, which had higher abundances of Epithemia spp. at most sites in 2005. This taxon includes five epithemiacean taxa (appendix table D2). The average difference was 16 percent higher in 2005; in the main stem downstream from Wright Creek (NUBWC) and upstream from Copeland Creek (COPEG), and in Steamboat Creek (STEAM) percent biovolume was substantially higher: $29-63$ percent. Of the 36 diatom taxa not observed in 1995, those with the highest percent biovolume ( $>25$ percent) in 2005 were Gomphoneis mammilla and Gomphoneis minuta. The average percent biovolume of Melosira varians was notably higher in 2005, constituting 13 and 50 percent, respectively, of the biovolume at LEM1T and TOKET, both downstream from reservoirs. Other differences included Gomphonema subclavatum (an oligotrophic and organic-enrichment-sensitive diatom), Rhopalodia gibba, Cymbella affinis, and Diatoma mesodon, which were all about 5 percent less in 2005 than in 1995.

For soft algae, the greatest difference between 1995 and 2005 was a 23 percent higher value for relative biovolume of Cladophora glomerata, a filamentous green alga. Higher Cladophora abundance coincided with higher percentages of certain diatom epiphytes including Epithemia spp., Cocconeis spp., and Achnanthidium minutissimum. In contrast, the percent biovolumes of Nostoc parmelioides, an $\mathrm{N}$-fixing blue-green alga, combined taxa in the $\mathrm{N}$ fixing Rivulariaceae (see footnote in appendix table D2), and two other genera of filamentous green algae (Mougeotia and Ulothrix) were 5-6 percent less in 2005 than 1995. Three other green algae (Gongrosira sp., Staurastrum punctulatum, and Spirogyra sp.) had smaller increases in 2005.
Species abundances within the restorationaffected reach only are shown in table 15.

\section{Changes in Algal Species Composition between 2005 and 2007}

Diatom species composition differed significantly between 2007 and 2005, with no overlap in the distribution of diatom samples among those years (fig. 20). The pattern was more complex for soft algae with clustering among LAKEM, LEM1G, and TOKLKI samples. A separate cluster was evident from the Wild and Scenic Reach (COPEG, NUSTM, NUBWC, and NURCR) in 2005, when the green algae Gongrosira sp. and blue-green Nostoc sp. were relatively more abundant.

Clustering differences in algal communities indicate changes in water quality and hydrology among the study years. The clusters of sites based on soft algae at main-stem sites in 2005 resulted from lower percent biovolume of Cladophora glomerata and Zygnema sp., two filamentous green algae. Other patterns may be explained by lower percent biovolume of $\mathrm{N}$-fixing diatoms in 2007 than 2005 at sites in the Wild and Scenic Reach (fig. 17) and throughout the restorationaffected reach (table 15). A small increase in available DIN during the drawdown and after the rotenone treatment may have lessened the competitive advantage of diatom $\mathrm{N}$-fixers. The percent biovolume of all eutrophic taxa 2007 was greater in 2007 than in 2005 in the lower Wild and Scenic Reach (fig. 17), with the largest increase in Nitzschia sp. and Cladophora glomerata (table 15). Percent biovolume of the diatom N-fixer Epithemia spp. was highest in 2005 (32 percent), and lower in 1995 (23 percent) and 2007 (17 percent). 
Table 15. Average percent biovolume of select algal taxa at sites extending from Lake Creek to the North Umpqua River above Rock Creek, 1995, 2005, and 2007.

[Taxa shown had the largest differences between sampling years. Taxon colors indicate green algae, blue-green algae, and diatoms. Indicator guilds defined in table 13. Abbreviations: --, not applicable or no data]

\begin{tabular}{|c|c|c|c|c|}
\hline Algal taxon & \multirow[t]{2}{*}{ Indicator guild(s) } & \multicolumn{3}{|c|}{$\begin{array}{c}\text { Average percent } \\
\text { biovolume for year }\end{array}$} \\
\hline 1995-2005 (pre-restoration) & & 1995 & 2005 & 2007 \\
\hline Cladophora glomerata & E, Alk-P & 0 & 23 & -- \\
\hline Epithemia spp. ${ }^{1}$ & $\mathrm{NF}$ & 23 & 32 & -- \\
\hline Spirogyra sp. & E, Alk-P & 0 & 4.4 & -- \\
\hline Closterium moniliferum & E, Alk-P & 0 & 4.4 & -- \\
\hline Gomphoneis minuta & E, OP-S & 0 & 3.6 & -- \\
\hline Filamentous blue-green algae ${ }^{2}$ & -- & 11 & 2.3 & -- \\
\hline Nostoc parmeloides & NF & 6.1 & 0.1 & -- \\
\hline Gomphonema subclavatum & $\mathrm{O}, \mathrm{OXY}-\mathrm{H}$ & 5.1 & 0.0 & -- \\
\hline Rivulariaceae & $\mathrm{NF}$ & 5.1 & 0.2 & -- \\
\hline Diatoma mesodon & M, OP-S, OXY-H & 4.7 & 0.0 & -- \\
\hline 2005-2007 & & 1995 & 2005 & 2007 \\
\hline Nitzschia sp. & -- & -- & 0.0 & 7.4 \\
\hline Cladophora glomerata & E, Alk-P & -- & 23 & 30 \\
\hline Filamentous blue-green algae $^{2}$ & -- & -- & 2.3 & 5.9 \\
\hline Eudorina elegans & $\mathrm{E}$ & -- & 0.0 & 2.4 \\
\hline Epithemia spp. ${ }^{1}$ & NF & -- & 32 & 17 \\
\hline Closterium moniliferum & E, Alk-P & -- & 4.4 & 0.0 \\
\hline Gomphoneis minuta & E, OP-S & -- & 3.6 & 0.6 \\
\hline Melosira varians & $\mathrm{E}, \mathrm{NH}$, Alk-P & -- & 3.5 & 0.6 \\
\hline
\end{tabular}

${ }^{1}$ Epithemia spp. includes Epithemia adnata, E. goeppertiana, E. sorex, E. turgida, and E. turgida var. westermannii.

${ }^{2}$ Filamentous blue-green algae includes Phormidium autumnale, P. sp., Leptolyngbya sp., Lyngbya martensiana, Homoeothrix janthina, $H$. juliana, $H$. varians, and Oscillatoria sp. 
Algal Conditions at Selected Sites from 1995 to 2005-07

Algal conditions were examined in greater detail at LAKEM, COPEG, and NURCR to evaluate the effect of the Diamond Lake restoration project. These sites were selected because they represent critical locations to evaluate the restoration project and its effect on the Wild and Scenic Reach.

LAKEM-Chl- $a$, AFDM, and total algal biovolume at LAKEM were much higher in 2005 than 1995 (photograph 9) (fig. 21), possibly due to the occurrence of algal blooms in Diamond Lake and associated $\mathrm{N}$ fixation, or other changes in growing conditions in that year. The percent biovolume of oligotrophic diatoms was 82.3 percent in 1995 but less than 10 percent in 200507 (table 14). Total biovolume of the large stalked diatom Gomphoneis herculeana, which was not observed in Lake Creek in 1995 or 2005, was substantially higher in 2006 and 2007 (appendix table D3), forming a glistening golden mat over many of the cobbles (photograph 10).

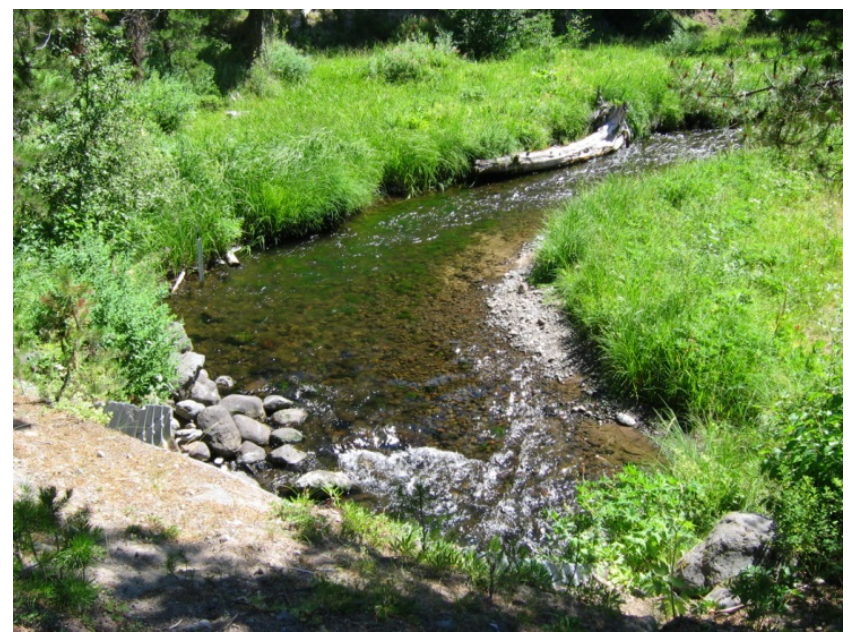

Photograph 9. Growths of filamentous green algae (Cladophora) in Lake Creek near the mouth, at LAKEM. (Photograph by Richard Grost, PacifiCorp, July 29, 2005.)
The change in oligotrophic diatoms at LAKEM between 2005 and 2007 is partly due to higher amounts of Achnanthidium minutissimum, an epiphytic diatom that commonly attaches to stalks of Gomphoneis (Loren Bahls, University of Montana, written commun., 2011). The total biovolume of many taxa were higher in 2005 than 1995, including Cladophora glomerata and two types of N-fixing algae, Nostoc parmelioides and Epithemia spp. Total algal biovolume, density, and Chl- $a$ was higher at LAKEM during the Diamond Lake drawdown in 2006 because of greater abundances of Cladophora glomerata and the diatom Stephanodiscus niagarae, a common planktonic diatom in Diamond Lake.

Total biovolume of filamentous blue-green algae, particularly Phormidium, also was much higher in 2006 and 2007 than in 1995 or 2005. Cladophora glomerata biovolume was substantially lower in 2007, with similar differences in Chl- $a$, and total algal biovolume (fig. 21). Epithemia spp. was not identified in the 2007 LAKEM sample (appendix table D3).

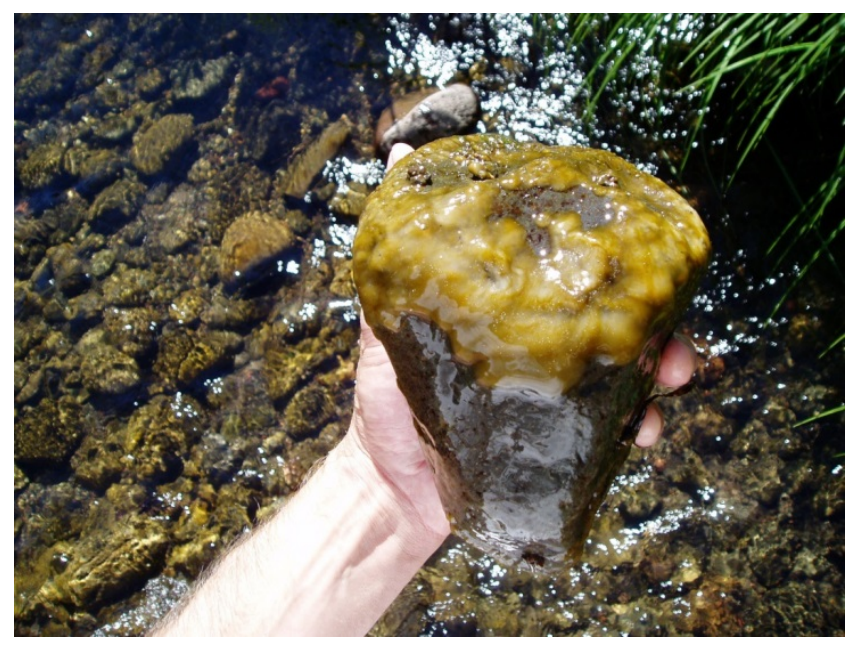

Photograph 10. Stalked diatoms (Gomphoneis herculeana) on cobble substrates in Lake Creek near the mouth, at LAKEM. (Photograph by Kurt Carpenter, U.S. Geological Survey, August 2, 2007.) 

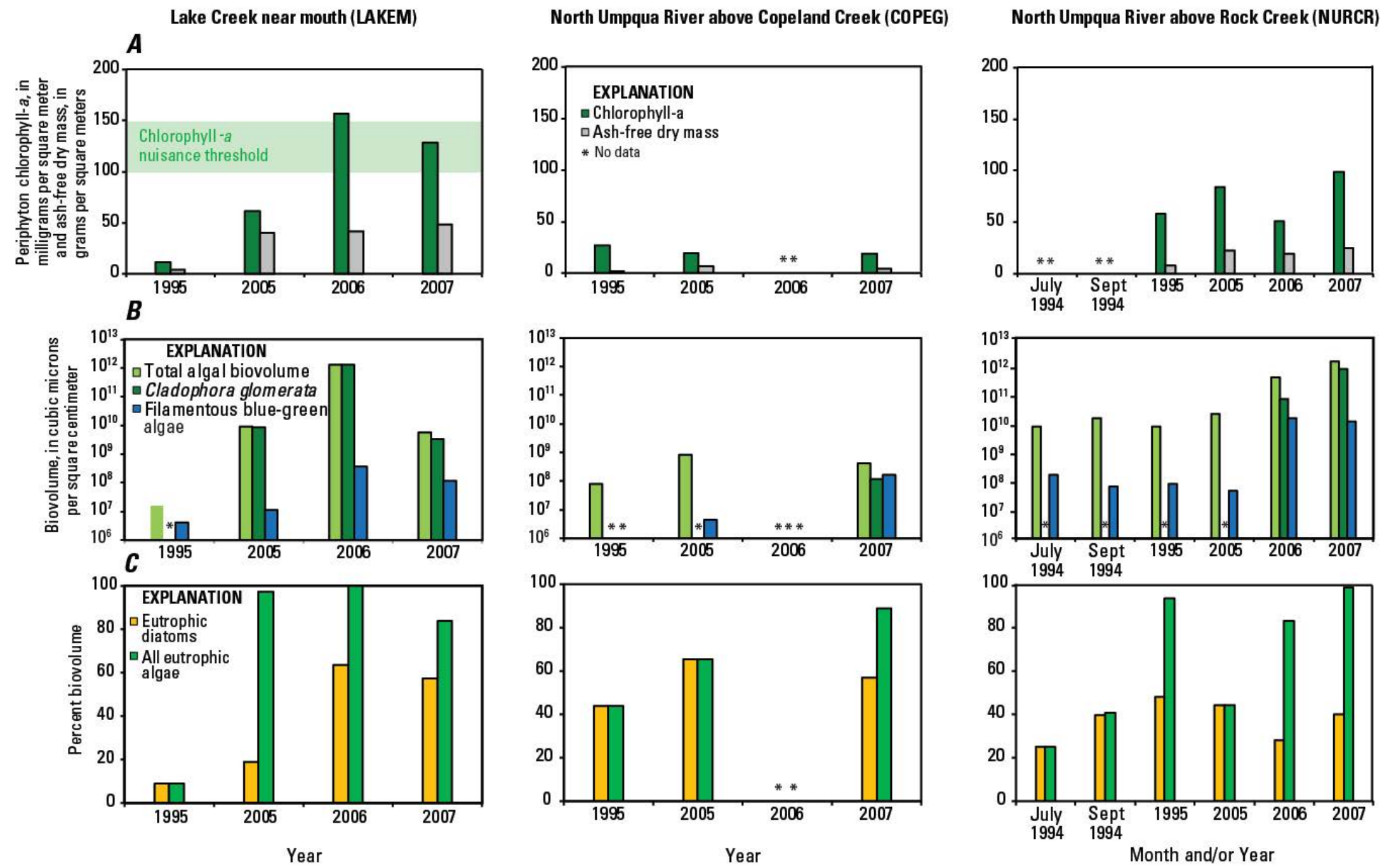

Figure 21. Algal conditions in Lake Creek and the North Umpqua River above Copeland and Rock Creeks, 1995, 2005-07, including (A) chlorophyll-a and ash-free dry mass, $(B)$ select algal biovolume indicators, and $(C)$ percent biovolume of eutrophic algae. Log scale in y-axis of plot $B$. Nuisance chlorophyll-a threshold range of 100-150 $\mathrm{mg} / \mathrm{m}^{2}$ suggested by Welch and others $(1988,1989)$. 
COPEG-Habitat conditions at this site are somewhat different from other main-stem sites, with greater water depth, higher water velocity, larger substrate size, and more shade. Algal conditions at COPEG were less variable than other sites, with only minor variations in Chl- $a$ among years (fig. 21). The algal cell density was lower in 2005, despite an increase in biovolume, than in 1995, which was mainly attributed to Epithemia spp. In 2005, percent biovolume of eutrophic taxa was higher than 1995, and higher still in 2007. Although Epithemia spp. and to a lesser degree Cocconeis sp. dominated the algal assemblages at COPEG in 2005 and 2007, filamentous green algae, Zygnema sp. and Gongrosira sp., were also abundant (table 12).

NURCR - In 1995, Zygnema sp. and Epithemia spp. constituted the largest percent biovolume at NURCR. In 2005, however, periphyton biomass was higher (fig. 21), owing to a higher percent biovolume of Epithemia spp. and Spirogyra, another type of filamentous green algae. Consequently, total cell density was lower in 2005 than 1995, reflecting an overall increase in the average cell size at this site. In 2006-07, two other types of filamentous green algae, Cladophora glomerata and Zygnema sp., and the diatom Nitzschia sp. accounted for the large increase in total biovolume and total cell density (table 12 and appendix table D3). Total biovolume of green algae and percent biovolume of eutrophic algae were higher in 2006 and 2007 than in 1995; lower values in 2005 (fig. 21) may have been related to reduced flows that year.

\section{Factors Shaping Periphyton Assemblages: Results} of Multivariate BEST Analyses

PRIMER-E BEST multivariate analyses were performed on the percent biovolume algal datasets from 1995, 2005, and 2007 to test whether streamflow, nutrients, and diel minimums and maximums of field-parameter values were significant in explaining patterns in the periphyton species composition. Separate analyses were performed on the diatoms and softalgae datasets (table 16). In order to isolate the effect of the Diamond Lake drawdown, only those sites within the restoration-affected reach were included in this analysis.

In all years, streamflow was the single most important variable in the BEST analysis solutions for diatoms and soft algae $(\mathrm{P}<0.05)$. In about half of the solutions, other variables, including maximum water temperature, TP, SRP, $\mathrm{NO}_{3}$, and specific conductance joined streamflow as significant, but secondary factors (table 16). In 2007, only streamflow was significant. 
Table 16. Summary of BEST analysis solutions listing the measured variables that best explain patterns in periphyton assemblages for sites within the Diamond Lake Restoration Affected Reach extending from Lake Creek to the North Umpqua River above Rock Creek, 1995, 2005, and 2007.

[Bolded values significant at $\mathrm{P}<0.05$. Analyses were conducted separately on diatoms and soft algae, with and without streamflow. Analysis was based on percent biovolume and species composition. BEST variables are those included in the final analysis solution. For convenience, soft algae analyses are shaded grey.]

\begin{tabular}{|c|c|c|c|c|c|c|c|c|}
\hline \multirow[b]{2}{*}{ Year } & \multirow[b]{2}{*}{$\begin{array}{c}\text { BEST } \\
\text { analysis } \\
\text { solution }\end{array}$} & \multirow[b]{2}{*}{$\begin{array}{l}\text { Algal } \\
\text { group }\end{array}$} & \multirow[b]{2}{*}{$\begin{array}{l}\text { Streamflow } \\
\text { included? }\end{array}$} & \multirow[b]{2}{*}{ BEST variables } & \multirow[b]{2}{*}{$\begin{array}{l}\text { Individual } \\
\text { variable } \\
\text { correlation }\end{array}$} & \multicolumn{3}{|c|}{ BEST solution sample statistics } \\
\hline & & & & & & $\begin{array}{c}\text { Overall } \\
\text { rho } \\
\text { value }\end{array}$ & $\begin{array}{c}\text { Significance } \\
\text { level }\end{array}$ & $\begin{array}{c}\text { Number } \\
\text { of } \\
\text { samples }\end{array}$ \\
\hline \multirow{11}{*}{1995} & 1 & Diatoms & Yes & Streamflow & 0.740 & 0.83 & $\mathbf{P}=0.002$ & 7 \\
\hline & & & & Total phosphorus & 0.681 & & & \\
\hline & & & & $\begin{array}{l}\text { Maximum water } \\
\text { temperature }\end{array}$ & 0.548 & & & \\
\hline & & & & Nitrate & 0.501 & & & \\
\hline & 2 & Diatoms & No & $\begin{array}{l}\text { Maximum water } \\
\text { temperature }\end{array}$ & 0.548 & 0.78 & $P=0.008$ & 7 \\
\hline & & & & Nitrate & 0.501 & & & \\
\hline & 3 & Soft algae & Yes & Streamflow & 0.756 & 0.84 & $P=0.003$ & 7 \\
\hline & & & & Total phosphorus & 0.632 & & & \\
\hline & & & & $\begin{array}{l}\text { Maximum water } \\
\text { temperature }\end{array}$ & 0.615 & & & \\
\hline & 4 & Soft algae & No & $\begin{array}{l}\text { Soluble reactive } \\
\text { phosphorus }\end{array}$ & 0.665 & 0.80 & $\mathbf{P}=\mathbf{0 . 0 0 3}$ & 7 \\
\hline & & & & $\begin{array}{l}\text { Maximum water } \\
\text { temperature }\end{array}$ & 0.615 & & & \\
\hline \multirow{8}{*}{2005} & 5 & Diatoms & Yes & Streamflow & 0.748 & 0.80 & $\mathrm{P}=0.001$ & 9 \\
\hline & & & & $\begin{array}{l}\text { Maximum water } \\
\text { temperature }\end{array}$ & 0.325 & & & \\
\hline & 6 & Diatoms & No & Nitrate & 0.603 & 0.74 & $\mathbf{P}=\mathbf{0 . 0 0 5}$ & 9 \\
\hline & & & & $\begin{array}{l}\text { Maximum water } \\
\text { temperature }\end{array}$ & 0.325 & & & \\
\hline & 7 & Soft algae & Yes & Streamflow & 0.495 & 0.50 & $P=0.001$ & 9 \\
\hline & 8 & Soft algae & No & Specific conductance & 0.341 & 0.37 & $\mathrm{P}=0.09$ & 9 \\
\hline & & & & Nitrate & 0.299 & & & \\
\hline & & & & $\begin{array}{l}\text { Maximum water } \\
\text { temperature }\end{array}$ & -0.004 & & & \\
\hline \multirow{4}{*}{2007} & 9 & Diatoms & Yes & Streamflow & 0.749 & 0.75 & $P=0.001$ & 9 \\
\hline & 10 & Diatoms & No & $\begin{array}{l}\text { Maximum water } \\
\text { temperature }\end{array}$ & 0.656 & 0.66 & $\mathrm{P}=0.233$ & 9 \\
\hline & 11 & Soft algae & Yes & Streamflow & 0.337 & 0.34 & $P=0.042$ & 9 \\
\hline & 12 & Soft algae & No & Specific conductance & 0.111 & 0.11 & $\mathrm{P}=0.278$ & 9 \\
\hline
\end{tabular}




\section{Discussion}

\section{Effects of the Diamond Lake Restoration Project on Lake Creek and the North Umpqua River}

The Diamond Lake restoration project affected water quality by producing elevated concentrations of organic carbon, nitrogen, and phosphorus in Lake Creek during the 2006 drawdown, and after flow resumed from the lake in 2007. Before the restoration project, Diamond Lake was already known to export significant nutrient loads to Lake Creek (Anderson and Carpenter, 1998). From 2001 to 2006, these loads could have been increasingly derived from Anabaena blooms (table 1). In 2005, prior to the restoration project, the $\mathrm{NO}_{3}$ concentration in Lake Creek was more than three times greater than in July 1995, and considerably greater than for all other streams sampled.

Eilers and others (2003) outlined the various natural and anthropogenic nutrient sources to Diamond Lake in a detailed nutrient budget, which was included in the TMDL for the lake (Oregon Department of Environmental Quality, 2006). Nitrogen enters Diamond Lake from the two southern tributaries, Short and Silent Creeks, primarily in the form of $\mathrm{NO}_{3}$ (Raymond, 2006). Anoxia in the lake sediments results in higher $\mathrm{NH}_{4}$ and SRP in the hypolimnion during summer, and this internal loading is enhanced by $\mathrm{N}$-fixing blue-green algae (Eilers and others, 2003).

Residual nitrogen from Diamond Lake flows into Lake Creek as organic nitrogen and $\mathrm{NH}_{4}$ (Hofford, 2004). The well-oxygenated waters of Lake Creek aid in the decomposition of organic matter and nitrification of $\mathrm{NH}_{4}$, resulting in higher concentrations of $\mathrm{NO}_{3}$ downstream at LAKEM (Hofford, 2004; Raymond, 2006; this study). Periodically, concentrations of $\mathrm{NO}_{3}$ in Lake Creek correspond to the intensity of the blue-green algal bloom in Diamond Lake, indicating that $\mathrm{N}$-fixing algae blooms in the lake may be an important source of $\mathrm{NH}_{4}$ and $\mathrm{NO}_{3}$ to
Lake Creek. For example, the intensity of bluegreen algal blooms in 2001 was much greater than in 2005, which was represented in the corresponding Lake Creek $\mathrm{NO}_{3}$ concentrations $(0.139 \mathrm{mg} / \mathrm{L}$ compared to about $0.025-0.033$ $\mathrm{mg} / \mathrm{L}$ ) (Hofford, 2004).

Blue-green algae blooms of various sizes occurred from 2001 to 2006, which includes the year of the rotenone treatment. The blooms increased Chl- $a$, decreased Secchi disk visibility, and increased concentrations of TP, $\mathrm{NH}_{4}$, and $\mathrm{NO}_{3}$ in the lake (Raymond, 2006. After 2001, $\mathrm{NH}_{4}$ concentrations in Diamond Lake increased and peaked in 2003, consistent with active $\mathrm{N}$ fixation (Raymond, 2005). These results are consistent with the TMDL for Diamond Lake; Eilers and others (2003) noted that Diamond Lake exports much more nitrogen than it receives through precipitation, tributaries, and anthropogenic inputs, and ascribed the additional nitrogen to $\mathrm{N}$-fixation by blue-green algae.

Future monitoring could track total and dissolved nitrogen exports from Diamond and Lemolo Lakes, and examine the their role in providing nutrients for proliferation of attached periphytic algae in Lake Creek and the Wild and Scenic Reach downstream.

Increases in biologically available nitrogen and phosphorus in Lake Creek contributed to higher algal biomass (Chl- $a$ and AFDW), higher algal biovolume, and increased proportion of eutrophic algae (fig. 21), especially the filamentous green alga Cladophora glomerata (photograph 9). This species responds to nitrogen and phosphorus enrichment and contributes to high mid-day $\mathrm{pH}$ values during the growing season. Previous reports have documented growths of high-biomass-forming filamentous green algae and associated high $\mathrm{pH}$ in similarly sized Cascade rivers, including the South Umpqua (Tanner and Anderson, 1996), Clackamas (Carpenter, 2003), and North Santiam Rivers (U.S. Geological Survey, 2013). 
In 2006 and 2007, the total biovolume of stalked diatoms, in particular Gomphoneis herculeana, increased markedly, possibly in response to elevated concentrations of dissolved organic nutrients from Diamond Lake. In addition to tolerating very cold water, these organisms produce copious amounts of extracellular mucilage in the form of polysaccharide stalks that attach cells to rocks and other surfaces. These stalks may serve as substrata for other, smaller, attached diatoms such as Achnanthidium spp. Stalks of other diatoms, for example Didymosphenia geminata contain enzymes that enable these taxa to metabolize organic nutrients from the water (Elwood and Whitton, 2007), but this has not yet been demonstrated for the stalked diatoms identified in the North Umpqua River Basin. An abrupt increase in the biovolume of Gomphoneis herculeana in Lake Creek in response to the Diamond Lake drawdown in 2006 suggests that this taxon might respond similarly. However, Steamboat Creek, a site without a perceptible spike in organic matter, also had a large increase in Gomphoneis herculeana between 2005 and 2007, so other factors probably are involved. Microscopic examination of the stalks of Cymbella from the Clackamas River, another Cascade Range stream, revealed epiphytic cells of the N-fixing diatom Epithemia on the surface of the Cymbella stalks. This raises the possibility that the stalked diatoms might be involved in a commensal or symbiotic relationship with Nfixing diatoms. It is possible that nitrogen fixed by the blue-green endosymbionts inside Epithemia somehow is transferred to the Cymbella stalk, and (or) that Epithemia receives some of the phosphorus liberated by enzymes within the stalks. This relationship, if verified, could explain the response of stalked diatoms in the North Umpqua River to increases in dissolved organic nutrients.

Because of their ability to produce mats of mucilage as much as 20 -cm thick, stalked diatoms can have significant biological effects on stream ecosystems by altering food web structure and hydraulic conditions (Spaulding and Elwell, 2007). If these algae become abundant, they could impact riffles that fish use for spawning, and degrade the health of rivers like the North Umpqua. Large masses of mucilage help these stalked diatoms withstand high water velocities and survive partial desiccation. Management strategies to control their spread include educational efforts that encourage careful washing of wader boots or prohibit the use of felt-soled waders in order to minimize the translocation of stalked diatoms and other nuisance species.

\section{Streamflow, Water Quality, and Algal Conditions between Synoptic Surveys}

Water quality and associated changes noted in this report between surveys in 1995 and 2005-2007 represent relatively few, individual points in time and therefore do not formally represent "trends". Data from the surveys are, however, useful to help characterize inter-annual variability as a way to understand how flow and various water quality parameters affect periphyton abundance and species composition. To facilitate comparisons among years, the synoptic surveys were conducted at or near the peak in annual maximum water temperatures, which also align with the annual maximum $\mathrm{pH}$ and minimum DO (fig. 6). Nevertheless difference in streamflow among years had effects on dilution rates and nutrient concentrations, and their combined effect on periphyton assemblages is complex.

Furthermore, there are regional processes that are consistent with some of the changes described. For instance, reductions in annual streamflow have been noted in southern Oregon between $\sim 1950$ and 2007, an analysis that includes the COPEG streamgage record (Mayer and Naman, 2011). To the extent that streamflow is a critical driver of water quality through dilution rates among other influences, these reductions could be due in part to changes in algal populations observed in our surveys. 
Similarly, the increasing frequency and intensity of Cyanobacterial blooms in Diamond and Lemolo Lakes prior to 2005 likely contributed to progressive eutrophication downstream. For the purposes of the discussion that follows, we consider changes among water quality constituents or algal metrics between survey dates to be generally indicative of ongoing processes unless otherwise stated, recognizing that data gaps remain, especially between 1995 and 2005. Additional data collection in future years would help to confirm whether any of these changes continue to occur, and could therefore be characterized more properly as trends.

1995-2005-Inter-annual variation in snowpack can substantially affect groundwater and melt-water-driven streamflow in the North Umpqua River and other Cascade rivers. Waterquality and algal conditions, in turn, are closely linked to surface-water hydrology. In a given year, streamflow has effects on physical and chemical habitat such as available substrate for periphyton to colonize; light for photosynthesis, which is mediated by turbidity, channel form, and depth; abrading physical scour, mediated by velocity and suspended sediment load (Francoeur and Biggs, 2006); and nutrient concentrations that can be diluted by flow. The 1995 snowpack in the North Umpqua River watershed was about six times larger than 2005 (Natural Resources Conservation Service, 2008) and explains some of the differences in streamflow, water quality, and algal conditions observed between these years. Streamflow averaged 25 percent less and water temperatures (diel minima and maxima) were higher in the Wild and Scenic Reach during the 2005 synoptic than in 1995 (fig. 6).

Concentrations of $\mathrm{NH}_{4}, \mathrm{NO}_{3}, \mathrm{SRP}$, and TP in the Wild and Scenic mainstem averaged 3350 percent higher in 2005 than in 1995 (table 10). As discussed previously, the greater frequency of $\mathrm{NO}_{3}$ detections in 2005 might have been due to differences in sample processing and preservation (see Methods section) or data reporting by the laboratories. If non-detections were assigned values equal to the laboratory reporting level (and not one-half the reporting level), then the change in $\mathrm{NO}_{3}$ in the Wild and Scenic Reach would be just 2 percent. However the $\mathrm{NO}_{3}$ concentrations in the tributaries entering the Wild and Scenic Reach were considerably higher in 2005, and provide a plausible source of $\mathrm{NO}_{3}$ to the mainstem.

Estimated nutrient loads identified some of the major nitrogen contributors in the watershed. During 1995, 2005, and 2007, total loading from the major tributaries to the Wild and Scenic Reach (Boulder, Copeland, and Steamboat Creeks, and Little River, among others) contributed 17, 9, and 15 percent of the DIN load in each year, as measured at Glide. Rock Creek was not sampled in 2007 but contributed 7-8 percent in 1995 and 2005. The pattern for TN loading was similar, with about 21,7 , and 12 percent of the main-stem load coming from the tributaries in 1995, 2005, and 2007. Rock Creek contributed 6-9 percent in the years it was measured. Meanwhile, upper-basin sources (as measured at BSODA), contributed 75 to 90 percent of the main-stem DIN loads at Glide. Of the upstream sources, INLET and LAKEM combined provided roughly 600-1,000 percent of the DIN load at Glide. Thus, there is a net reduction in DIN load through the Wild and Scenic Reach, presumably from uptake by periphyton and other retention processes in the river, reservoirs, and forebays.

Periphyton biomass was higher-by as much as 60 percent — at the two sites downstream of Steamboat Creek (NUSTM and NURCR) in 2005 compared with 1995. In 2005, algal species assemblages had higher abundance of filamentous green algae and nitrogen-fixing diatoms and less filamentous blue-green algae. The algal species NMDS ordinations showed much dissimilarity between the 1995 and 2005 samples, due in part to differences between contractor taxonomy and enumeration protocols (see "Methods" section). If the species shifts in 2005 are, however, real, they indicate a change 
toward a more eutrophic river with more highbiomass-forming filamentous green algae with nitrogen-fixing epiphytes to sustain its growth.

2005-2007-Changes in North Umpqua River streamflow from 2005 to 2007 were partially the result of normal hydrologic variation and partially from the Diamond Lake restoration project. Streamflow in the Wild and Scenic Reach at COPEG was lower than normal in 2005, but increased in 2006 as a result of a large snowpack and the additional 22,000 acre- $\mathrm{ft}$ of water released from Diamond Lake during the drawdown. Flows were also somewhat lower than normal in spring 2007 as Diamond Lake refilled. Late summer base flow in the Wild and Scenic Reach was about the same in all years because groundwater dominates flows from the upper basin and the hydroelectric project essentially discharges natural baseflow without any net storage or reduction during the summer.

Maximum water temperatures at the NURCR monitor were similar from year to year and exceeded the ODEQ standard of $16^{\circ} \mathrm{C}$ (based on the 7-day running average of the daily maximum temperature) on 61-65 days per year in 2005-07. An unusual summer storm and cooler air temperatures in 2007 contributed to cooler water temperatures during the August 2007 sampling (fig. 6). Tributary temperatures, including those in the Western Cascades along the Wild and Scenic Reach, were warmer than main-stem temperatures (fig. 5).

Despite the cooler conditions, the daily maximum $\mathrm{pH}$ was notably higher during the 2007 synoptic sampling compared with other years, with the highest values occurring downstream of Toketee Lake at TOKET and the main stem upstream of Rock Creek at NURCR (fig. 5). The daily maximum $\mathrm{pH}$ in the North Umpqua River near Idleyld Park also was highest in 2007 (fig. 6). Issues with the continuous $\mathrm{pH}$ data in 2005 precluded midsummer comparisons with 2007. The $\mathrm{pH}$ exceeded the ODEQ standard of 8.5 units during most of July through September in 2007, but exceeded the standard for just a few days in 2006. Because of its low alkalinity and buffering capacity, the North Umpqua River is particularly sensitive to the effect that algal photosynthesis has on inorganic carbon and $\mathrm{pH}$. Unlike water temperature, which is a function of many factors (solar input, streamflow, water source, weather, and shading), variations in $\mathrm{pH}$ directly reflect primary production by aquatic plants in this system.

The Diamond Lake restoration had pronounced effects on nutrients, particularly during 2006, when a pulse of DIN made its way through Lake Creek, the hydroelectric project, and the Wild and Scenic Reach downstream to NURCR. In 2006, a smaller pulse was observed for DOC, and phosphorus contributions from Diamond Lake were small compared to the normal background signal from groundwater. Nutrient concentrations during the 2007 synoptic survey were slightly elevated over 2005, including DIN, TKN, SRP, and TP. Whether this represented a residual from the restoration project in 2007 or another source is unknown.

The periphyton response to the Diamond Lake restoration project was not consistent. Although biomass in the Wild and Scenic Reach was similar in 2005 and 2007, changes in species made the overall community compositions distinct in 2005 (fig. 20). In the upper part of the Wild and Scenic Reach at COPEG, where habitat for algae is determined largely by available light and substrate and the temperatures are cool, algal conditions showed little variability from year to year. Downstream from Steamboat Creek, the percent abundance of filamentous-green algae increased 2007, whereas the percentage of abundance of $\mathrm{N}$-fixing diatoms decreased, possibly reflecting higher DIN concentrations. These changes are also consistent with the $\mathrm{pH}$ increases observed in 2007 at NURCR. Stalked diatoms, meanwhile, increased substantially from 2005 to 2006 and 2007 in Lake Creek, again potentially indicative of the export of dissolved organic material and nutrients from Diamond Lake. 
Because algal samplings are just a snapshot of conditions during summers, however, it is unclear whether the observed differences between years represent a real change in the assemblages or are artifacts of sampling during different stages of succession. Caution is warranted when interpreting patterns in water quality and algal conditions among sites or years. For this reason, multiple lines of evidence, including algal biomass, species composition, and guild structure, were evaluated alongside water-quality and streamflow data to support hypotheses and conclusions about river conditions.

\section{Potential Effects of Resource Management on Water Quality and Algal Conditions}

Anderson and Carpenter (1998) presented an analysis of the factors controlling water quality in the North Umpqua River Basin, including the role of resource management, and suggested studies that could refine their conclusions and provide tools to minimize negative effects on water quality. The findings of this report largely confirm the overall findings from Anderson and Carpenter (1998). Some external factors have also changed the way the basin has been managed since 1995 and present some potential threats to water quality.

Forestry and hydroelectric power generation continue to be the principal resource management activities in the North Umpqua River Basin. Aside from some water-quality issues in Diamond and Lemolo Lakes, development and recreational use in the basin appear to have had a limited effect on water quality. Important changes in the watershed since 1995 include reduced logging on Federal and private lands, but that trend appears to be slowly reversing. In that time, wildfire has killed trees on more acres than have been harvested in the North Umpqua watershed (Steve Nelson, U.S. Forest Service, Pacific Northwest Region, written commun., 2013).
Mountain pine beetle (Dendroctonus ponderosae) is an emerging threat to upper-basin forests and may already be causing negative effects on water quality. The beetle has affected 400 acres of lodgepole pine in the Diamond Lake-Lemolo Lake drainage and in some areas of Crater Lake National Park (Donald J. Goheen, U.S. Forest Service Entomologist-Plant Pathologist, written commun., 2008). In northern Colorado, there is an apparent causal link between beetles and short-term increases in streamflow, and increases in stream concentrations of TOC, DOC, and P. Stednick and Jensen (2007) found that mountain pine beetle damage to northern Colorado lodgepole pine forests was correlated with higher concentrations of $\mathrm{NO}_{3}$ in surface water. In contrast, Lukas and Gordon (2010) reported that soil nitrogen increased in beetle-infested forests, but that export of nitrogen to streams was either small or nonexistent. Beetle damage leads to decaying foliage and wood, reduced root uptake of nutrients and water, and increased soil temperature and moisture, resulting in higher water yields and mobilization of nitrogen as decomposition products stimulate nitrifying bacteria in groundwater and surface water. The higher nitrogen load at INLET in 2005, despite lower streamflow (fig. 16), suggests an increase in nitrogen mobilization and export from the forest. It is possible that beetle infestation in the High Cascades is responsible for at least some of the observed increase in $\mathrm{NO}_{3}$ concentrations in the upper North Umpqua River at INLET from 1995 to 2005. A well-designed sampling program that examines $\mathrm{NO}_{3}$ concentrations in forest soils, groundwater, and surface water in affected and unaffected areas of the basin would help scientists and land managers better understand beetle infestation effects on nitrate loading to the watershed.

From 1995 to 2005, other changes and activities in the basin have likely had effects on water quality. The terms of the new FERC license for the hydroelectric project have restricted power peaking, increased minimum 
instream flows in the bypass reaches, and reduced winter drawdown of Lemolo Lake. The effect of these changes is complex. For example, increased bypass flows may have contributed to reduced periphyton biomass downstream from Lemolo Lake in 2007 through increased scour. At the same time, the higher bypass flows may have changed the mixing in the lake during summer, which, under some conditions, could enhance blue-green algae blooms and nitrogen fixation, contributing to higher nitrogen concentrations downstream. This hypothesis could be investigated by numerical modeling approaches, with targeted sampling to verify results.

The large blooms of Anabaena in Diamond and Lemolo Lakes (Eilers and others, 2003; Eilers, 2008) present a challenge for land, water, and fisheries managers. Anabaena blooms are a potential health hazard, and, along with other Nfixing algae, could provide nitrogen that fuels periphyton growth in the main stem. Depending on the amount of nitrogen fixed and ultimately transported downstream, and how long it takes to "spiral" through the system, this nitrogen could stimulate further growths of algae downstream. The relatively short residence time in Lemolo Lake (15-22 days; Anderson and Carpenter, 1998) probably results in nitrogen export, from a summer bloom, in late fall or winter, after the peak algal growing season, but longer-term retention and release of nutrients from reservoir sediments may also be occurring. Diamond Lake, on the other hand, with a longer residence time of 1.6 years (Johnson and others, 1985), might export nitrogen over a longer period of time, especially after spring turnover, when periphyton is beginning to colonize and grow in the river. If this is occurring, then Diamond Lake nitrogen could contribute to higher algal biomass or shifts in species composition in Lake Creek and (or) downstream reaches. Information on site-specific processes, including the amount of nitrogen fixed in Diamond and Lemolo Lakes during blooms, and the fate of that nitrogen, are lacking. More study, therefore, is needed to fully evaluate the consequences of Anabaena blooms in these lakes to determine how they may be affecting algal and water-quality conditions downstream, particularly in the Wild and Scenic Reach.

\section{Suggestions for Future Monitoring and Studies}

Previous studies and the ongoing operation of continuous monitors in the North Umpqua River have produced a rich dataset that will enhance future interpretations and data collection activities in this watershed. Anderson and Carpenter (1998) suggested a range of potential studies to inform management of the river basin, including continued tracking of $\mathrm{pH}$ and $\mathrm{DO}$, and measurements of algal biomass to better define annual variability in algal conditions; measurements of algal productivity and nutrient uptake; experiments to define more specifically nutrient limitation; studies to evaluate the influence of geomorphology and hydrology on algal growth, productivity, population dynamics, and nutrient uptake; surveys of background nutrient contributions from sources including groundwater, lake sediments, and upland soils; lake and reservoir processing of nutrients; and food-web dynamics as potential factors influencing the ecology of the aquatic system.

Data collected since the 1995 survey have largely been focused on the FERC relicensing activities, and on algal blooms in Diamond and Lemolo Lakes, and the Diamond Lake restoration project. These studies advanced the general understanding of water quality in the river basin; however, many of the study topics proposed by Anderson and Carpenter (1998), though relevant, remain unexplored.

Ongoing Monitoring - Continued waterquality and algal monitoring would help identify possible future changes in river conditions and trends over time. Water quality and algal monitoring provide a baseline and context for additional data collection and management actions. They advance understanding of how the 
watershed functions and can be used to evaluate management practices. Continuous water-quality data can be used to gauge algal activity in real time. $\mathrm{pH}$ is directly indicative of primary production in the North Umpqua River because of the low alkalinity and limited buffering capacity of the North Umpqua River, and because of the slower equilibrium kinetics of $\mathrm{CO}_{2}$ dissolution into water (compared with dissolved oxygen; Guasch and others, 1998) makes $\mathrm{pH}$ a particularly good indicator of periphyton activity. As described above, large diel swings in $\mathrm{pH}$ are a common occurrence in the North Umpqua River during summer.

An important unanswered question for basin managers is whether nitrogen loading is increasing, as suggested by this study, regardless of the 2006 drawdown at Diamond Lake. Periodic monitoring during the growing season for nutrients, periphyton biomass, and algal species composition, combined with continuous $\mathrm{pH}$ and DO monitoring at key locations, could help answer this question. A monitoring program that includes the upper North Umpqua River at INLET, LAKEM, COPEG, and NURCR, and select tributaries representative of the major drainage types (Clearwater, Boulder, and Steamboat Creeks, for example) could track inputs to the Wild and Scenic Reach. This level of monitoring would shed light on the year-toyear and seasonal variations in growing conditions, and might reveal changes due to climate, weather, or changes in the management of the watershed. Periodic inclusion of benthic invertebrates, and especially the density and composition of algae grazers, an understudied component of the North Umpqua ecosystem, would further enhance knowledge of river algal conditions and benthic ecology. Continued monitoring in Diamond and Lemolo Lakes for nutrients, phytoplankton biomass, and species composition, and for limnological processes would help managers understand the nutrient dynamics involved and how they affect periphyton growth in the Wild and Scenic Reach of the North Umpqua River. This information would also help in better describing the seasonal and year-to-year variability in nutrients exported from these lakes as they relate, in particular, to the recurrent blooms of blue-green algae.

Anecdotal reports indicate long-term reductions in visual clarity in the river, and associated increases in algae on rocks that impair the scenic quality of the river, and particularly its legendary steelhead fishery. These changes could be a result of progressive eutrophication, with a resulting increase in algal particles in the water from sloughed periphyton or upstream phytoplankton blooms. Alternatively, reductions in visual clarity may be traceable to increased erosion, especially if fine or colloidal particles have increased from wildfire, beetle kills, or other sources. Ascribing causes to these changes such as construction of the hydroelectric project or commercial forestry is not possible because baseline data are lacking. Turbidity, which often is used to evaluate transport of particulates, is not a definitive technique here because suspended algal cells tend to be large compared to the wavelength of turbidity-meter light sources, and not reflective, and, therefore, scatter light only minimally. However, during critical periods, carefully conducted surveys for visual clarity using light-boxes (Davies-Colley and Smith, 2001), combined with detailed turbidity measurements could reveal differences in upstream-downstream clarity as well as appropriate uses of turbidity measurements. The collection of suspended algal cells using drift nets, and measurement of biomass along with identification of the species collected, would better document the role of algal drift in reducing visual clarity in the river, and would provide valuable information on the health of the North Umpqua River food web.

Technological advances, especially for continuous monitors (Pellerin and others, 2011), will also provide new opportunities for evaluating North Umpqua River water quality in coming years. Continuous fluorescence measurement of water column Chl- $a$ is part of USGS monitoring programs in some of Oregon's 
other Cascade rivers such as the Clackamas and North Santiam Rivers (U.S. Geological Survey, 2013). Episodic losses of periphyton

occasionally occur in these cold, high-gradient streams in response to sloughing events in late spring and summer or in response to changes in streamflow. Continuous monitoring of Chl- $a$, which is not yet part of the monitoring program, could record episodes of algal drift and clarify its relation with turbidity, streamflow changes, or other factors in the North Umpqua River.

Sensors for fluorescent dissolved organic matter (FDOM) also are available and could help detect changes in source waters (Downing and others, 2009; Carpenter and others, 2013), such as during flow manipulations within the hydroelectric project area or storm-runoff periods. Sensors for nitrate-N and phosphorus also are available, but detection levels are likely too high for rivers such as the North Umpqua River. Continued improvement may allow such sensors to collect meaningful data in the near future. The use of continuous nutrient sensors will revolutionize the understanding of nutrient dynamics in natural waters, potentially revealing diel patterns or relations to management actions that cannot be discerned with monthly or even synoptic sampling.

Nutrient Sources for Algae - As discussed by Anderson and Carpenter (1998), future studies could quantify nutrient sources in the basin, including decomposition and release of nutrients from organic matter in soils, $\mathrm{N}$-fixing algal blooms in basin lakes and reservoirs, and forest fertilization if it continues in the future (Anderson, 2001). Future studies could also explore potential contributions from forested areas affected by mountain pine beetles, wildfire, and atmospheric deposition of $\mathrm{NO}_{3}$ in snow (Yang and others, 1995). Future algal studies could shed light on the effect of high phosphorus concentrations on the abundance of and nitrogen inputs from benthic N-fixing diatoms (DeYoe and others, 1992). Targeted sampling of groundwater seepage in "gaining" reaches, coupled with pore-water sampling for dissolved nutrients prior to the growing season, could provide insights about upper-basin phosphorus and nitrogen sources and their effect on periphyton or phytoplankton growth in Lemolo and Diamond Lakes, Lake Creek, Clearwater River, and selected segments in the Wild and Scenic Reach.

\section{Stream Reach Metabolism and} Biogeochemical Mesocosm Studies-Although periphyton biomass and species composition are highly variable throughout the North Umpqua River Basin, interpretations based on water chemistry and species ecologies indicate that nitrogen may be an important driver of algal conditions. Because of the strong nutrient uptake potential, especially when periphyton reach nuisance biomass levels, innovative approaches may be needed to understand the processes in downriver nutrient transport, uptake, and recycling. Recent advances in tracer techniques using stable isotope injection can estimate nutrient uptake and release within a stream reach, also known as nutrient spiraling (Earl and others, 2007; Payn and others, 2005). Results from the Lotic Inter-site Nitrogen Experiment (LINX) suggest tight nutrient spiraling in streams where algae are strongly limited by nitrogen. Similar spiraling studies, along with nutrient addition experiments to test the nature (nitrogen versus phosphorus) and degree of potential limitation, would help resource managers establish targets to possibly reduce the occurrence of nuisance-level periphytic growths in the Wild and Scenic North Umpqua River.

$\mathrm{N}$-fixing algae are potentially a large source of nitrogen for the North Umpqua River. Nitrogen inputs from Anabaena and other Nfixers in Diamond and Lemolo Lakes could be quantified using microbial techniques (An and others, 2001). Coupled studies of nitrogen fixation, nitrification, and denitrification in the water column and sediments of Diamond and Lemolo Lakes could shed light on how nitrogen cycles within these lakes and may present management opportunities for improving water quality and algal conditions downstream. 
Effect of Streamflow on Algae-The multivariate analyses conducted for this study (table 16) indicated that streamflow was a key variable shaping benthic algae assemblages in the North Umpqua River Basin. Streamflow affects nutrient concentrations through dilution and controls available habitat for periphyton to attach and grow. FERC license conditions will have direct and indirect effects on periphyton and perhaps phytoplankton from increased flows in the bypass reaches, reduced stage fluctuations, and controlled ramping rates in Lemolo Lake and the Wild and Scenic Reach.

Using methods similar to Instream Flow Incremental Methodology studies for fish (U.S. Geological Survey, 2014b), studies could evaluate the effect of streamflow on the amount of habitat available for periphyton to colonize. An Instream Flow Incremental Methodology applied to periphyton could help managers understand how different water depths, velocities, and substrates affect periphytic growth and assemblage composition. Such a study might provide insights into why, for example, periphyton attains high biomass and produces very high $\mathrm{pH}$ in some years but not in others.

\section{Potential Effects of High pH on} Salmonids-High $\mathrm{pH}$ caused by algal photosynthesis has been observed in several reaches of the North and South Umpqua, Clackamas, and North Santiam Rivers. Phytoplankton blooms in the reservoirs and periphytic growths in flowing reaches contribute to high $\mathrm{pH}$. For weeks and sometimes months during the growing season, diel maximum $\mathrm{pH}$ values in the main-stem North Umpqua River exceed $8.5 \mathrm{pH}$ units and approach $9.5 \mathrm{pH}$ units in the main flow (thalweg) for a few hours each day when favorable conditions support high rates of algal photosynthesis.

This effect of photosynthesis on $\mathrm{pH}$, including the diel variations, is characteristic of the productive and poorly buffered waters found in many Cascade streams during spring and summer. The continuous water-quality monitor in the North Umpqua River near Idleyld Park indicates that during the past 10 years, diel maximum $\mathrm{pH}$ values have exceeded the ODEQ water-quality standard on nearly 40 percent of the days in June-August. It is likely that even higher $\mathrm{pH}$ values occur along stream margins where algal biomass is often concentrated, and where hydraulic flushing rates are lower. Strongly alkaline water has physiological effects on fish that may include reduced ability to regulate ion balance and excrete ammonia, effects that are manifested within fish gills (Laurent and others, 2000). Although no information is available for native salmonids, brook trout gill lamellae become injured at a $\mathrm{pH}$ of 9.0 units (Daye and Garside, 1976). The effect of diel, high $\mathrm{pH}$ on developing salmonid fry, although not unique to the North Umpqua River, has not been well documented, but could be investigated with laboratory or stream-side experiments. Such studies could identify thresholds in $\mathrm{pH}$ effects that possibly apply to local fish and could provide support for waterquality standards based on $\mathrm{pH}$.

\section{Conclusions}

This study evaluated the effects of the 2006 Diamond Lake restoration project on water quality and algal conditions in downstream reaches, particularly the Wild and Scenic Reach of the North Umpqua River. It used data collected from repeat surveys conducted in 1995, 2005, and 2007, as well as monthly samples from 2005-07, and continuous records of water temperature, $\mathrm{pH}$, and dissolved oxygen at several locations in the basin to evaluate changes over time.

Water-quality and biological changes were observed between the 1995 and 2005 surveys that were unrelated to the restoration project, but may be attributable to the same blue-green algae blooms that were the impetus for the restoration 
project in Diamond Lake. Similar blooms occurred periodically in Lemolo Lake, and when combined with changes in hydrology related to climatic patterns and forest management, contributed to higher nutrient loading to the North Umpqua River by 2005.

The restoration project included a 10-month drawdown of Diamond Lake preceding the application of rotenone to eliminate invasive Tui chub (Gila bicolor) and reset the lake's food web. As hypothesized, the restoration project significantly increased export of total and dissolved nitrogen and organic carbon from the lake into Lake Creek. The pulse of nutrients from Diamond Lake was observed in Lake Creek near its mouth, in the hydroelectric project at the inflow to Toketee Lake, and to a lesser degree, near the upstream boundary of the Wild and Scenic Reach upstream from Copeland Creek, and near the downstream terminus of the Wild and Scenic Reach near Idleyld Park.

Changes in algal biomass and community composition were also observed-between 1995 and 2005, and during the course of the restoration project at Diamond Lake. Increased availability of nitrogen and flow changes from the restoration project provide the most likely explanation for the observed increase in algal Chl- $a$ in the lower Wild and Scenic Reach in 2007. Additionally, the algal assemblages differed in each of the 3 years, with substantially higher biovolume of Cladophora in the latter years. Greater amounts of algal productivity were reflected in elevated $\mathrm{pH}$, particularly at the continuous water-quality monitor in the lower Wild and Scenic Reach near Idleyld Park.

Despite episodes of decreased water quality observed during this study, including high water temperatures, high $\mathrm{pH}$, and nuisance levels of algae, the effects of the nutrient pulse from the Diamond Lake restoration project are expected to be temporary. The extent to which these changes linger in the system will be partly dependent on the future of $\mathrm{N}$-fixing algal blooms in Diamond and Lemolo Lakes, and perhaps the response of other $\mathrm{N}$-fixing periphyton in the downstream system. Although an assessment of the success or failure of the rotenone application is beyond the scope of this study, nutrient exports from Diamond Lake decreased in 2007, and the monitoring in Diamond and Lemolo Lakes has continued. Cyanobacteria blooms in Diamond Lake were of shorter duration and less intense during the first few years following the restoration project.

The Wild and Scenic North Umpqua River remains one of the highest quality waters in the State of Oregon, supporting resident trout populations and runs of wild salmon and steelhead. For many years, large blooms of potentially toxic blue-green algae in Diamond and Lemolo Lakes have threatened water quality, fisheries, and public health, not just within those water bodies, but also in downstream reaches of the North Umpqua River. The primary bluegreen algae in those blooms, Anabaena, is a nitrogen fixer, and appears to have periodically enriched nitrogen levels in the system, setting off a chain of events - stimulating periphytic algae that in turn contribute to nuisance levels of biomass in the river that cause $\mathrm{pH}$ to increase sharply during daylight hours.

These symptoms of nutrient enrichment in North Umpqua River Basin waters have been apparent for more than a decade. If the river is to continue to support the many designated beneficial uses set in the Oregon statutes, efforts will be needed to reduce nutrient inputs, and restore natural processes that use and store nutrients to prevent their transport to surface waters. Future studies and monitoring would expand our understanding of nutrient dynamics and watershed processes in the basin and would better quantify the nutrient sources, including nitrogen fixed by algae, to guide management strategies that will improve water-quality conditions in this valued Wild and Scenic River. 


\section{Acknowledgements}

This report was prepared in cooperation with Douglas County with administrative and field support from Tom Manton, Jerre Woody, and Joe Cooper. Much appreciated field sampling assistance was provided by Joy Archuleta, Debra Gray, Kevin Hounshell, Al Johnson, Amy Rusk, and Craig Street (U.S. Forest Service), Kirk Haskett and Kevin Saylor (Oregon Department of Fish and Wildlife), Paul Heberling (Oregon Department of Environmental Quality), and Richard Grost (PacifiCorp). The authors gratefully acknowledge Gene Foster and Gregory Pettit from the Oregon Department of Environmental Quality for contributing to this study with funding and sample analyses.

\section{References Cited}

Aiken, G., L.A. Kaplan, and J. Weishaar, 2002, Assessment of relative accuracy in the determination of organic matter concentrations in aquatic systems: Journal of Environmental Monitoring, v. 4, p. 70-74.

An, S., Gardner, W.S., Kana, T., 2001, Denitrification and nitrogen fixation using isotope pairing with membrane inlet mass spectrometry analysis: Applied Environmental Microbiology, v. 67, no. 3, p. 1171-1178.

American Public Health Association (APHA), 1992, Standard Methods of Water and Wastewater, 18th edition: American Public Health Association, American Water Works Association, Water Environment Federation, Washington D.C.

Anderson, C.W., 2001, Ecological effects on streams from forest fertilization-Literature review and conceptual framework for future study in the western Cascades: U. S. Geological Survey Water-Resources Investigations Report 01-4047, 49 p., http://or.water.usgs.gov /pubs_dir/WRIR01-4047/
PacifiCorp generously provided facilities for field crews. Within the U.S. Geological Survey, Joanne Wallis, Marc Stewart, Micelis Doyle, Heather Bragg, Dwight Tanner (retired USGS), Steven Sobieszczyk, and Andrew Arnsberg (formerly with the USGS) contributed valuable assistance with field sampling, data compilation, and mapping. USGS volunteer Raychel Parks assisted with data collection and sample processing.

Anderson, C.W., and Carpenter, K.D., 1998, Water quality and algal conditions in the North Umpqua River basin, Oregon, 1992-95, and implications for resource management: U.S. Geological Survey Water-Resources Investigations Report 98-4125, 78 p., http://or.water.usgs.gov/pubs_dir/Abstracts/98 $-4125 . h t m l$

Bahls, L. L., 2004, Northwest Diatoms-A photographic catalogue of species in the Montana diatom collection, with ecological optima, associates, and distribution records for the nine northwestern United States, vol. 1.

Bahls, L. L., 2005, Northwest Diatoms-A photographic catalogue of species in the Montana diatom collection, with ecological optima, associates, and distribution records for the nine northwestern United States, vol. 1.

Bonoff, M.B., Mattax, B.L., and Olson, T.M., 1996, Diel Study of Lemolo Lake, Douglas County, Oregon: Northwest Science, v. 70, no. 2, p. 48-58.

Buchanan, T.J., and Somers, W. P., 1984, Discharge measurements at gaging stations: U.S. Geological Survey Techniques of WaterResources Investigations, book 3, chapter A8, 65 p., http://pubs.usgs.gov/twri/twri3a8

Burkholder, B.K., 2007, Influence of hyporheic flow and geomorphology on temperature of a 
large, gravel-bed river, Clackamas River, Oregon, USA: Oregon State University Master's Thesis, 179 p.

Caldwell, R.R., 1998, Chemical study of regional ground-water flow and groundwater/surface-water interaction in the upper Deschutes basin, Oregon: U.S. Geological Survey Water-Resources Investigations Report 97-4233, 49 p.

Carpenter, K.D., 2003, Water quality and algal conditions in the Clackamas River basin, Oregon, and their relations to land and water management: U. S. Geological Survey WaterResources Investigations Report 2002-4189, 114 p., http://pubs.usgs.gov/wri/WRI02-4189

Carrick, H.J., Lowe, R.L., and Rotenberry, J.T., 1988, Guilds of benthic algae along nutrient gradients - Relationships to algal community diversity: Journal of the North American Benthological Society, v. 7, no. 2, p. 117-128.

Cazaubon, A., Rolland, T., and Loudiki, M., 1995, Heterogeneity of periphyton in French Mediterranean rivers: Hydrobiologia, vol. 300/301, p. 105-114.

Charles, D.F., Knowles, C., and Davis, R.S., 2002, Protocols for the analysis of algal samples collected as part of the U.S. Geological Survey National Water-Quality Assessment Program: The Academy of Natural Sciences Patrick Center for Environmental Research-Phycology Section Report No. 02$06,124 \mathrm{p}$., http://water.usgs.gov/nawqa/protocols/algprot ocol/index.html

Clarke, K.R. and Gorley, R.N., 2006, PRIMER v6, User Manual/Tutorial: Primer-E, Plymouth, UK, 190 p.

Davies-Colley, R.J., and Smith, D.G., 2001, Turbidity, suspended sediment, and water clarity_A review: Journal of the American Water Resources Association, v. 37, no. 5, p. 1085- 1101

Daye, P.G., and Garside, E.T., 1976, Histopathologic changes in surficial tissues of brook trout, Salvelinus fontinalis (Mitchill), exposed to acute and chronic levels of $\mathrm{pH}$ : Canadian Journal of Zoology, v. 54, p. 21402155.

Downing, B.D., Boss, E., Bergamaschi, B.A., Fleck, J.A., Lionberger, M.A., Ganju, N.K., Schoellhamer, D.H., and Fujii, R., 2009, Quantifying fluxes and characterizing compositional changes of dissolved organic matter in aquatic systems in situ using combined acoustic and optical measurements: Limnology and Oceanography Methods, v. 7, p. 119-131.

Earl, S., Valett, H.M., and J.R. Webster, 2007, Comparison of nitrogen spiraling in stream ecosystems quantified using stable isotope tracers and nutrient addition experiments: Limnology and Oceanography vol. 52, p. 1718-1723.

Edwards, T.K. and Glysson, D.G., 1999, Field methods for measurement of fluvial sediment: U.S. Geological Survey Techniques of WaterResources Investigations, book 3, chapter c2, 89 p., http://pubs.usgs.gov/twri/twri3c2/html/pdf.html

Eilers, J.M., 2008, Water Quality in Diamond Lake During 2006 and 2007; submitted to Oregon Department of Fish and Wildlife: Bend, Oregon, $40 \mathrm{p}$.

Eilers, J.M., Eilers, B.J., and Kann, J., 2003, TMDL modeling and analysis of Diamond Lake, Oregon: Bend, Oregon, J.C. Headwaters, Inc., 103 p., accessed October 18, 2006 at http://www.fs.usda.gov/Internet/FSE_DOCUM ENTS/stelprdb5336128.pdf

Eilers, J.M., Loomis, D., St. Amand, A., Vogel, A., Jackson, L., Kann, J., Eilers, B., Truemper, H., Cornett, J., and Sweets, R., 2007, Biological effects of repeated fish introductions in a formerly fishless lake: Diamond Lake, Oregon, USA: Fundamental and Applied Limnology / Archiv für Hydrobiologia, v. 169, no. 4, p. 265-277. http://www.dfw.state.or.us/fish/local_fisheries/ diamond_lake/docs/Eilers_2007_Fund_Appl_ Limnology.pdf, accessed October 16, 2013. 
Eilers, J.M., and Truemper, H., 2010, Diamond Lake Recovery_Again: Lakeline, North American Lake Management Society, summer 2010, p. 23-26.

Eilers, J.M., Truemper, H., Jackson, L.S., Eilers, B.J., and Loomis, D.W., 2011, Eradication of an invasive cyprinid (Gila bicolor) to achieve water quality goals in Diamond Lake, Oregon (USA): Lake and Reservoir Management, v. 27, p. 194-204.

Elwood, N.T.W. and Whitton, B.A., 2007, Importance of organic phosphate hydrolyzed in stalks of the lotic diatom Didymosphenia geminata and the possible impact of atmospheric and climatic changes: Hydrobiologia, v. 592, p. 121-133.

Federal Energy Regulatory Commission, 2003, Order Approving Settlement Offer and Issuing New License, PacifiCorp Project No. 1927008, Washington, DC., 106 pp.

Francoeur, S.N. and Biggs, B.J.F., 2006, Shortterm effects of elevated velocity and sediment abrasion on benthic algal communities: Hydrobiologia, vol. 561, p. 59-69.

Franken, R.J., Storey, R.G., and Williams, D.D., 2001, Biological, chemical and physical characteristics of downwelling and upwelling zones in the hyporheic zone of a northtemperate stream: Hydrobiologia, vol. 444, p. 183-1951.

Guasch, H., Armengol, J., Marti, E., and Sabater, S., 1998, Diurnal variation in dissolved oxygen and carbon dioxide in two low-order streams: Water Research, v. 32, no. 4, p. 1067-1074.

Harza Engineering Co., Inc.(now MWH Global, Inc.), 1999, Flow, pH, and nutrient study, North Umpqua Hydroelectric Project, FERC Project No. 1927, prepared for PacifiCorp: Broomfield, Colorado, 29 p. and appendices.

Herrett, T.A., Hess, G.W., Stewart, M.A., Ruppert, G.P., and Courts, M.L., 2005, Water Resources Data for Oregon, Water Year 2005: U.S. Geological Survey Water Data Report OR-05-01, http://pubs.usgs.gov/wdr/2005/wdror-05
Hofford, S., 2004, Report on streamflow regime, stream water quality, channel morphology, and related aquatic conservation strategy, November 22, 2004: http://www.fs.usda.gov/Internet/FSE_DOCUM ENTS/stelprdb5336130.pdf, accessed October 16, 2013.

Jefferson, A., Grant, G., and Lewis, S., 2007, A river runs underneath it - Geological control of spring and channel systems and management implications, Cascade Range, Oregon, in M.J. Furniss, C.F. Clifton, and K.L. Ronnenberg, eds., Advancing the Fundamental Sciences, Proceedings of the Forest Service national earth sciences conference, San Diego, California, October 18-22, 2004: Portland, Oregon, U.S. Department of Agriculture, Forest Service, Pacific Northwest Research Station, p. 391-400.

Johnson, D.M., Peterson, R.R., Lycan, D.R., Sweet, J.W., Neuhaus, M.E., and Schaedel, A.L., 1985, Atlas of Oregon Lakes: Corvallis, Oregon State University Press, 318 p.

Jones, M., Eilers, J., and Kann, J., 2007, Water quality effects of blue-green algal blooms in Diamond Lake, Oregon, in Furniss, M., Clifton, C., and Ronnenberg, K., eds., Advancing the Fundamental Sciences, Proceedings of the Forest Service National Earth Sciences Conference, San Diego, California, October 18-22, 2004: U.S. Department of Agriculture, Forest Service, Pacific Northwest Research Station, p. 102110.

Kennedy, E.J., 1983, Computation of continuous records of streamflow: U.S. Geological Survey Techniques of Water-Resources Investigations, book 3, chapter a13, 53 p., http://pubs.usgs.gov/twri/twri3-a13/.

Laurent, P., Wilkie, M.P., Chevalier, C., Wood, C.M., 2000, The effect of highly alkaline water (pH 9.5) on the morphology and morphometry of chloride cells and pavement cells in the gills of the freshwater rainbow trout-Relationship to ionic transport and ammonia excretion: 
Canadian Journal of Zoology, v. 78, p. 307319.

Lowe, R.L., and Pan, Y., 1996, Benthic algal communities as biological monitors, in Stevenson, J.R., Bothwell, M.L., and Lowe, R.L., eds., Algal ecology, freshwater benthic ecosystems: San Diego, California, Academic Press, Inc., p. 705-739.

Lukas, J. and Gordon, E., 2010, Impacts of the mountain pine beetle infestation on the hydrologic cycle and water quality - A symposium report and summary of the latest science: Intermountain west climate summary, a product of the Western Water Assessment, v. 6, no. 4, p. 1-6, URL: http://wwa.colorado.edu/climate/iwcs/docs/20 10_May/IWCS_May2010_Feature.pdf

Mayer, T.D., and Naman, S.W., 2011, Streamflow response to climate as influenced by geology and elevation: Journal of the American Water Resources Association, v. 47, doi: 10.1111/j.1752-1688.2011.00537.x, accessed October 16, 2013.

McFarland, W.D., 1983, A description of aquifer units in western Oregon: U. S. Geological Survey Open-File Report 82-165, 35 p.

Moffatt, R.L., Wellman, R.E., and Gordon, J.M., 1990, Statistical summaries of streamflow data in Oregon: Volume 1-Monthly and annual streamflow, and flow duration values: U.S. Geological Survey Open-File Report 90-118, $413 \mathrm{p}$.

Moulton, S.R., Kennen, J.G., Goldstein, R.M., and Hambrook, J.A., 2002, Revised protocols for sampling algal, invertebrate, and fish communities as part of the USGS National Water-Quality Assessment Program: U.S. Geological Survey Open File Report 02-150, $75 \mathrm{p}$.

Natural Resources Conservation Service, 2008, SNOTEL data from NRCS web site at URL: ftp://ftp-

fc.sc.egov.usda.gov/OR/snow/snowcourse/or_d ata/V_NORTHUMPQUA.txt, accessed

December 10, 2008.
Natural Resources Conservation Service, 2013, Air temperature data from Oregon (PST) SNOTEL site Toketee Airstrip (1044) (22F45S), at http://www.wcc.nrcs.usda.gov/nwcc/view, accessed March 20, 2013.

Oregon Department of Environmental Quality,1996, Oregon Draft 1994/1996 list of water quality limited water bodies - 303(d)(1): Portland, Oregon, [variously paged].

Oregon Department of Environmental Quality, 1998, Laboratory field sampling reference guide, Rev. 6.0: Portland, Oregon, 55 p.

Oregon Department of Environmental Quality, 2004, Laboratory quality manual, Version 5: Portland, Oregon, DEQ91-LAB-0006-QMP, $63 \mathrm{p}$.

Oregon Department of Environmental Quality, 2006, Total maximum daily loads for the Umpqua basin, Chapter 6 (plus appendices) Diamond Lake and Lake Creek aquatic weeds, dissolved oxygen, and $\mathrm{pH}$ : http://www.deq.state.or.us/WQ/TMDLs/docs/ umpquabasin/umpqua/chpt6dialake.pdf, accessed December 13, 2010.

Oregon Department of Environmental Quality, 2009, Water Monitoring and Assessment Mode of Operations Manual (MOMs): Portland, Oregon, 90 p., http://www.deq.state.or.us/ lab/techrpts/docs/DEQ03LAB0036SOP.pdf, accessed January 18, 2014).

Oregon Department of Environmental Quality, 2010, Water Quality Assessment-Oregon's 2010 integrated report assessment database and 303(d) list: http://www.deq.state.or.us/wq/assessment/rpt2 010/search.asp, accessed July 16, 2012

Oregon Department of Human Services, 2010, Harmful algae bloom surveillance program web site: http://www.oregon.gov/DHS/ph/hab/ index.shtml, accessed November 3, 2010.

PacifiCorp, 1995, Application for new license for major modified project-Final technical report for water quality, North Umpqua 
Hydroelectric Project, FERC Project No. 1927:

Portland, Oregon, PacifiCorp, Volumes 1-42, [variously paged].

PacifiCorp, 1998, The North Umpqua

cooperative watershed analyses-

Supplemental material for the synthesis report, Volume III, North Umpqua Hydroelectric Project, FERC Project No. 1927, Douglas

County, Oregon: Portland, Oregon, PacifiCorp, [variously paged].

Payn, R. A., Webster, J. R., Mulholland, P. J., Valett, H. M., and Dodds, W. K., 2005, Estimation of stream nutrient uptake from nutrient addition experiments: Limnology and Oceanography Methods vol. 3, p. 174-182.

Pellerin, B.A, Bergamaschi, B.A, Murdoch, P.S, Downing, B.D, Saraceno, J, Aiken, GR, and Striegl, RG. 2011, The aquatic real-time monitoring network; in-situ optical sensors for monitoring the nation's water quality: U.S. Geological Survey Fact Sheet 2011-3061, 2 p., http://pubs.usgs.gov/fs/2011/3061/

Porter, S.D., 2008, Algal attributes: An autecological classification of algal taxa collected by the National Water- Quality Assessment Program: U.S. Geological Survey Data Series 329, http://pubs.usgs.gov/ds/ds329/.

Powell, M.A., 1995, Report on $\mathrm{pH}$ in the Jackson Creek and Little River drainage basins of the Umpqua National Forest: Roseburg, Oregon, U.S. Department of Agriculture, Forest Service, Umpqua National Forest, Report to the U.S. Forest Service, [variously paged]. [Available from Supervisor, Umpqua National Forest, Roseburg, OR 97471]

Power, M.E., Parker, M.S., and Dietrich, B.E., 2008, Seasonal reassembly of a river food web-Floods, droughts, and impacts on fish: Ecological Monographs, v. 78, no. 2, p. 263282.

Rantz, S.E., and others, 1982, Measurement and computation of streamflow, Volumes 1 and 2: U.S. Geological Survey Water-Supply Paper
2175, 631 p., http://pubs.usgs.gov/wsp/wsp2175.

Raymond, R., 2006, Water-quality monitoring of Diamond Lake for 2005, Report prepared for the Umpqua National Forest, Roseburg, Oregon, 91 p. [Available from Supervisor, Umpqua National Forest, Roseburg, OR 97471]

Rinella, F.A., 1998, Major-ion, nutrient, and trace-element concentrations in the Steamboat Creek basin, Oregon, 1996: U.S. Geological Survey Water-Resources Investigations Report 98-4105, 31 p., http://or.water.usgs.gov/pubs_dir/Abstracts/ 98-4105.html

Rinella, J.F., 1979, Lakes of Oregon, Volume 6, Douglas County: U.S. Geological Survey Open-File Report, 124 p.

Risley, J. C., 2004, Floods of November 1996 through January 1997 in the Umpqua River basin, Oregon: U.S. Geological Survey Fact Sheet 2004-3134, http://pubs.usgs.gov/fs/2004/3134/

Salinas, J.T., and Larson, D.W., 1995. Diamond Lake-Limnological and bacterial investigations, 1992-1994: Roseburg, Oregon, U.S. Forest Service, Umpqua National Forest, Final Report, Umpqua National Forest, CAS9501, [variously paged].[ Available from Supervisor, Umpqua National Forest, Roseburg, OR 97471]

Snyder, D.T., and Morace, J.L., 1997, Nitrogen and phosphorus loading from drained wetlands adjacent to Upper Klamath and Agency Lakes, Oregon: U.S. Geological Survey WaterResources Investigations Report 97-4059, 67 p., http://pubs.er.usgs.gov/usgspubs/wri/ wri974059

Spaulding, S.A. and Elwell, L., 2007, Increase in nuisance blooms and geographic expansion of the freshwater diatom Didymosphenia geminate-Recommendations for response: U.S. Geological Survey Open-File Report 2007-1425, 38 p., http://www.fs.usda.gov/Internet/FSE_DOCUM 
ENTS/fsbdev3_015176.pdf, accessed October 16, 2013.

Stednick, J.D. and Jensen, R., 2007, Effects of pine beetle infestations on water yield and water quality at the watershed scale in northern Colorado-Report as of FY2007 for USGS State Water Resources Research Institute Program, Grant 2007CO153B: http://water.usgs.gov/wrri/07grants/ 2007CO153B.html.

Stevenson, R.J., Bothwell, M.L., and Lowe, R.L., 1996, Algal ecology — Freshwater benthic ecosystems: San Diego, California, Academic Press, Inc., 753 p.

Stevenson, R.J., 1997, Resource thresholds and stream ecosystem sustainability: Journal of the North American Benthological Society, v. 16, no. 2, p. 410-424.

Sytsma, M., Miller, R., and Petersen, R., 2007, Diamond Lake water quality monitoring 2006-Data quality assessment, database description, and water-quality assessment, Report prepared for the U.S. Forest Service: 36 p., http://www.fs.usda.gov/Internet/FSE_DOCUM ENTS/stelprdb5336164.pdf, accessed December 15, 2010.

Sytsma, M., and Miller, R., 2008, Diamond Lake water quality during 2007 and long term trends of fish stocking index parameters-Report prepared for the U.S. Forest Service: 44 p., http://www.fs.usda.gov/Internet/FSE_DOCUM ENTS/stelprdb5336097.pdf, accessed December 15, 2010.

Sytsma, M., and Miller, R., 2011, Diamond Lake water quality assessment-2010 health monitoring index, Prepared for the Partnership for Umpqua Rivers, USDA Umpqua National Forest and the Oregon Department of Environmental Quality: Portland State University, $17 \mathrm{p}$.

Tague, C., and Grant, G.E., 2004, A geological framework for interpreting the low-flow regimes of Cascade streams, Willamette River Basin, Oregon: Water Resources Research, v.
40, no. 4, p. W04303, 9 p. http://dx.doi.org/10.1029/2003WR002629, accessed August 1, 2014.

Tanner, D.Q., Arnsberg, A.J., Anderson, C.W., and Carpenter, K.D., 2006, Water Quality and Algal Data for the North Umpqua River basin, Oregon, 2005: U.S. Geological Survey Data Series 229, p., http://pubs.usgs.gov/ds/2006/229/

U.S. Environmental Protection Agency, 1983, Methods for chemical analysis of water and wastes: Report 600/4-79-020, Revised 3/83, $552 \mathrm{p}$.

U.S. Forest Service, 2004, Diamond Lake restoration project-Final environmental impact statement: Roseburg, Oregon, U. S. Forest Service, Umpqua National Forest, http://www.fs.usda.gov/detail/umpqua/landma nagement/projects/?cid=stelprdb5336043, accessed October 18, 2006.

U. S. Forest Service, 2001, Middle North Umpqua Watershed Analysis: U. S. Forest Service, Umpqua National Forest, Roseburg, Oregon:

http://www.fs.usda.gov/detail/umpqua/landma nagement/?cid=stelprdb5335945, accessed July 16, 2012

U.S. Forest Service, 2009, FY 2008 monitoring report, Umpqua National Forest: Roseburg, Oregon, $50 \mathrm{p}$., http://www.fs.usda.gov/Internet/FSE_DOCUM ENTS/fsbdev3_056038.pdf, accessed October 12, 2011.

U.S. Geological Survey, 1915, Profile surveys in 1914 in Umpqua River Basin, Oregon: U.S. Geological Survey Water-Supply Paper 379, 7 p. +13 maps.

U.S. Geological Survey, 1992, Office of Water Quality Technical Memorandum 93.04, 4 p., http://water.usgs.gov/admin/memo/QW/

U.S. Geological Survey, 2013, USGS Data Grapher and Data Tabler: http://or.water.usgs.gov/grapher/.

van Dam, H., Mertens, A., and Sinkeldam, J., 1994, A coded checklist and ecological 
indicator values of freshwater diatoms from the Netherlands: Netherlands Journal of Aquatic Ecology, v. 28, no. 1, p. 117-133.

Wagner, R.J., Boulger, R.W., Jr., Oblinger, C.J.,, and Smith, B.A., 2006, Guidelines and standard procedures for continuous waterquality monitors-Station operation, record computation, and data reporting: U.S. Geological Survey Techniques and Methods 1-D3, 51 p. plus attachments, http://pubs.usgs.gov/tm/2006/tm1D3/pdf/TM1 D3.pdf.

Welch, E.B., Horner, R.R., Patmont, C.R., 1989, Prediction of nuisance periphytic biomass-A management approach: Water Resources, $\mathrm{v}$. 23 , no. 4, p. 401-405.

Welch, E.B., Jacoby, J.M., Horner, R.R., and Seeley, M.R., 1988, Nuisance biomass levels of periphytic algae in streams: Hydrobiologia, v. 157, p. 161-168.

Wetzel, R.G., 1983, Limnology (Second ed.): Philadelphia, CBS College Publishing, 767 p. + appendices.
Wallick, J.R., O'Connor, J.E., Anderson, S., Keith, M., and Cannon, C., and Risley, J.C., 2011, Channel change and bed-material transport in the Umpqua River basin, Oregon: U.S. Geological Survey, 110 p., http://pubs.usgs.gov/sir/2011/5041/

Wilde, F.D., Radtke, D.B., Gibs, J., and Iwatsubo, R.T., 1999, Collection of water samples: U.S. Geological Survey Techniques of Water-Resources Investigations Book 9, chapter A4, http://pubs.water.usgs.gov/twri9A4/.

Zarnetske, J.P., Haggerty, R., Wondzell, S.M., and Baker, M.A., 2011, Dynamics of nitrate production and removal as a function of residence time in the hyporheic zone: Journal of Geophysical Research-Biosciences, v. 116, no. G1, p. G01025, http://onlinelibrary.wiley.com/doi/10.1029/201 0JG001356/abstract. 


\section{On-Line Appendixes}

Available at: $h t t p: / / p u b s . u s g s . g o v / o f / 2014 / 1098 /$

\section{Appendix A. Laboratory Detection Limits and Reporting Levels}

Table A1. Methods of water quality and algal analysis for samples from the North Umpqua River basin, Oregon, 2005-07.

\section{Appendix B. Quality-Assurance Data}

Table B1. Quality-assurance results for nutrients and carbon in field blank samples, North Umpqua River basin, Oregon, 2005-07.

Table B2. Quality-assurance results for nutrients and carbon in replicate samples, North Umpqua River basin, Oregon, 2005-07.

Table B3. Quality-assurance results for the standard reference sample for nutrients.

Table B4. Quality-assurance results for replicate periphyton biomass samples for the North Umpqua River above Rock Creek (NURCR), North Umpqua River basin, Oregon, 2005-07.

Table B5. Quality-assurance results for triplicate algal biomass samples from the North Umpqua River basin, Oregon, 2005-07.

Table B6. Quality-assurance results for replicate algal species identification samples for the North Umpqua River above Rock Creek (NURCR), North Umpqua River basin, Oregon, 2005-07.

Table B7. Quality-assurance results (percent similarity) for replicate algal species identification samples for the North Umpqua River above Rock Creek (NURCR), North Umpqua River basin, Oregon, 2005-07.

\section{Appendix C. Water Quality Data from 2005-07}

Table C1. Censored water quality data for sites sampled during the synoptic events in the North Umpqua River basin, Oregon, 1995-07.

Table C2. Censored water quality data for the four sites sampled monthly in the North Umpqua River basin, Oregon, 2005-07.

\section{Appendix D. Algal Species Assemblage Data from 2005-07}

Table D1. Periphyton Chl-a, pheophytin-a, and ash-free dry mass from the North Umpqua River basin, Oregon, 1995-2007.

Table D2. Cell densities of algal taxa collected from sites in the North Umpqua River basin, Oregon, 1994-2007.

Table D3. Biovolumes of algal taxa collected from sites in the North Umpqua River basin, Oregon, 1994-2007.

Table D4. Spearman rank correlations between algal guilds or major Divisions and select environmental variables for samples collected in the North Umpqua River basin, Oregon, 1995-2007. 

